\title{
Estudo qualitativo de um modelo de propagação de dengue
}

\author{
Bruna Cassol dos Santos
}

\author{
DisSERTAÇÃO APRESENTADA \\ $\mathrm{AO}$ \\ Instituto De Matemática e Estatística \\ DA \\ Universidade DE SÃo PaUlo \\ PARA \\ OBTENÇÃO DO TÍTULO \\ DE \\ Mestre em CiÊnCIAS \\ Programa: Matemática Aplicada \\ Orientadora: Prof. Dr ${ }^{\mathrm{a}}$. Joyce da Silva Bevilacqua
}

Durante o desenvolvimento deste trabalho a autora recebeu auxílio financeiro do CNPq 


\section{Estudo qualitativo de um modelo de propagação de dengue}

Esta versão da dissertação contém as correções e alterações sugeridas pela Comissão Julgadora durante a defesa da versão original do trabalho, realizada em 25/07/2016. Uma cópia da versão original está disponível no

Instituto de Matemática e Estatística da Universidade de São Paulo.

Comissão Julgadora:

- Prof ${ }^{a}$. Dra ${ }^{a}$. Joyce da Silva Bevilacqua - IME-USP

- Prof. Dr. Sergio Muniz Oliva Filho - IME-USP

- Prof. Dr. Eduardo Massad - FM-USP 


\section{Agradecimentos}

A minha família: minha mãe Glória, meu pai Dijalma e meu irmão Gian pelo carinho e incentivo dedicados a mim durante todo este período. Por serem pacientes e pelo apoio incondicional na busca pelos meus objetivos. Tenho muito orgulho de ser filha de vocês e vos dedico esta conquista.

A Belmiro Galo da Silva pessoa que admiro muito, por estar ao meu lado em todos os momentos, por sua amizade, pelos risos e por todo o seu amor. Você é um grande companheiro!

Agradeço a minha orientadora professora Joyce, por ter me aceito como orientanda e por sua amizade. Pela sua paciência, seus ensinamentos e por todo o seu incentivo para que eu pudesse vivenciar todas as experiências que a vivência acadêmica pode porporcionar.

Agradeço aos professores e funcionários do IME, em especial aos professores: Saulo Rabello Maciel de Barros, André Salles de Carvalho, Sergio Oliva, Claudia Peixoto, Nelson Kuhl e Pedro Peixoto pelas discussões e valiosas contribuições durante a construção deste trabalho.

Agradeço a todos os colegas e ex-colegas do IME: Larissa, Lorena, Simone, Pedro, Guilherme, Marline, Anderson, Diego, Benigno, André, Wiliam, Carla, Flávia, Valdir, que constribuiram com o meu crescimento acadêmico e pessoal.

Às amigas Silva e Maria Leidiana pela parceria incondicional, pelas conversas, pelas risadas e por se tornarem parte da família que fiz aqui.

À minha mãe do coração Clarice pela sua amizade e por ter me acolhido com tanto carinho em sua família.

Agradeço ao CNPq pelo apoio financeiro. 


\section{Resumo}

DOS SANTOS, B. C. Estudo qualitativo de um modelo de propagação de dengue. 2016. 103 f. Dissertação (Mestrado) - Instituto de Matemática e Estatística, Universidade de São Paulo, São Paulo, 2016.

Em epidemiologia matemática, muitos modelos de propagação de doenças infecciosas em populações têm sido analisados matematicamente e aplicados para doenças específicas. Neste trabalho um modelo de propagação de dengue é analisado considerando-se diferentes hipóteses sobre o tamanho da população humana. Mais precisamente, estamos interessados em verificar o impacto das variações populacionais a longo prazo no cálculo do parâmetro $R_{o}$ e no equilíbrio endêmico. Vamos discutir algumas ideias que nortearam o processo de definição do parâmetro $R_{o}$ a partir da construção do Operador de Próxima Geração. Através de um estudo qualitativo do modelo matemático, obtivemos que o equilíbrio livre de doença é globalmente assintoticamente estável se $R_{o} \leq 1$ e instável se $R_{o}>1$. Para $R_{o}>1$, a estabilidade global do equilíbrio endêmico é provada usando um critério geral para estabilidade orbital de órbitas periódicas associadas a sistemas autônomos não lineares de altas ordens e resultados da teoria de sistemas competitivos para equações diferenciais ordinárias. Também foi desenvolvida uma análise de sensibilidade do $R_{o}$ e do equilíbrio endêmico com relação aos parâmetros do modelo de propagação. Diversos cenários foram simulados a partir dos índices de sensibilidade obtidos nesta análise. Os resultados demostram que, de forma geral, o parâmetro $R_{o}$ e o equilíbrio endêmico apresentam considerável sensibilidade a taxa de picadas do vetor, $b$ e a taxa de mortalidade do vetor, $\mu_{v}$

Palavras-chave: Epidemiologia matemática, modelos epidemiológicos, dengue, número de reprodutibilidade basal, equilíbrio endêmico, estabilidade global, análise de sensibilidade. 


\section{Abstract}

DOS SANTOS, B. C. Qualitative study of a dengue disease transmission model. 2016. 103 f. Dissertação (Mestrado) - Instituto de Matemática e Estatística, Universidade de São Paulo, São Paulo, 2016.

In mathematical epidemiology many models of spread of infectious diseases in populations have been analyzed mathematically and applied to specific diseases. In this work a dengue propagation model is analyzed considering different assumptions about the size of the human population. More precisely, we are interested to verify the impact of population long-term variations in the calculation of the parameter $R_{o}$ and endemic equilibrium. We will discuss some ideas that guided the parameter setting process $R_{o}$ from the construction of the Next Generation Operator. Through a qualitative study of the mathematical model, we found that the disease-free equilibrium is globally asymptotically stable if $R_{o} \leq 1$ and unstable if $R_{o}>1$. For $R_{o}>1$ the global stability of the endemic equilibrium is proved using a general criterion for orbital stability of periodic orbits associated with non-linear autonomous systems of higher orders and results of the theory of competitive systems for ordinary differential equations. Also a sensitivity analysis of the $R_{o}$ and the endemic equilibrium with respect to the parameters of the propagation model was developed. Several scenarios were simulated from the sensitivity index obtained in this analysis. The results demonstrate that in general the parameter $R_{o}$ and the endemic equilibrium are the most sensitive to the vector biting rate, $b$ and the vector mortality rate, $\mu_{v}$.

Keywords: Mathematical epidemiology, epidemiological models, dengue, basic reproduction number, endemic equilibrium, global stability, sensitivity analysis. 


\section{Sumário}

Lista de Símbolos

Lista de Figuras $\quad$ xiii

Lista de Tabelas $\quad$ xv

Introdução $\quad$ xvii

1 Fenômenos epidemiológicos e a modelagem matemática $\quad 1$

1.1 Motivação . . . . . . . . . . . . . . . . . . . . . 1

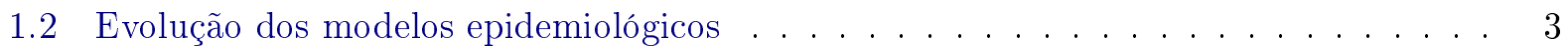

1.3 Aspectos teóricos dos modelos de propagação de epidemias . . . . . . . . . . . . 6

1.4 Parâmetros epidêmicos . . . . . . . . . . . . . . . . 6

1.4 .1 Força de infecção, $\lambda \ldots \ldots \ldots \ldots \ldots \ldots$

1.4.2 Número de Reprodutibilidade Basal, $R_{o} \ldots \ldots \ldots \ldots \ldots \ldots$

1.4.3 Formalização do conceito de $R_{o}$ baseado na teoria demográfica . . . . . . . 8

1.4.4 Formalização do conceito de $R_{o}$ a partir de um modelo epidêmico . . . . . . . 9

1.4.5 Formalização do conceito de $R_{o}$ para uma população estruturada . . . . . . . 11

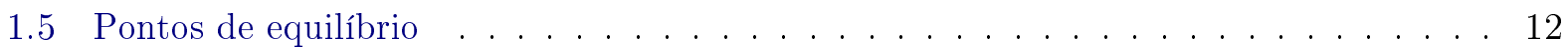

2 Estudo Qualitativo de Modelos de Propagação do Dengue 13

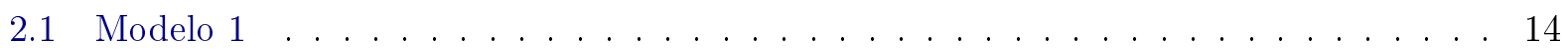

2.1 .1 Descrição do modelo matemático . . . . . . . . . . . . . . . . . 14

2.1.2 Pontos de equilíbrio e Número de Reprodutibilidade Basal . . . . . . . . . . . 17

2.1.3 Análise de estabilidade dos pontos de equilíbrio . . . . . . . . . . . . . . . 18

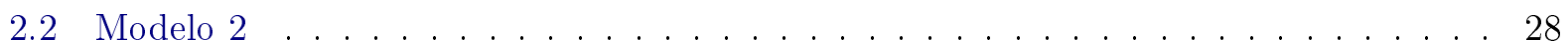

2.2 .1 Descrição do modelo matemático . . . . . . . . . . . . . . . 28

2.2.2 Pontos de equilíbrio e Número de Reprodutibilidade Basal . . . . . . . . . . . 30

2.2 .3 Análise de estabilidade dos pontos de equilíbrio . . . . . . . . . . . . . . 33

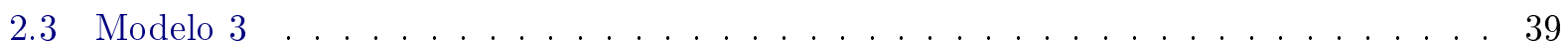

2.3 .1 Descrição do modelo matemático . . . . . . . . . . . . . . . . . . 39

2.3.2 Pontos de equilíbrio e Número de Reprodutibilidade Basal . . . . . . . . . . . 42

2.3.3 Análise de estabilidade dos pontos de equilíbrio . . . . . . . . . . . . . . . . 44

2.4 Comentários . . . . . . . . . . . . . . . . . . . . . . 54 
3 Resultados Numéricos $\quad 57$

3.1 Métodos numéricos ........................... 57

3.2 Comportamento assintótico ........................... 58

3.3 Parâmetro $R_{O}$.............................. 61

3.4 Análise de sensibilidade . . . . . . . . . . . . . . . . . . . . . . . 62

3.4.1 Descrição da análise de sensibilidade . . . . . . . . . . . . . . . . . . . 62

3.4.2 Índices de sensibilidade para o $R_{o}$. . . . . . . . . . . . . . . . . . 63

3.4.3 Índices de sensibilidade para o equilíbrio endêmico . . . . . . . . . . . . . . . 64

$\begin{array}{lll}\text { A Definições Preliminares } & 75\end{array}$

A.1 Noções de Estabilidade . . . . . . . . . . . . . . . . . . . . . . . . . . . . . 75

A.2 Resultados da teoria de sistemas competitivos e estabilidade de órbitas periódicas . . 76

$\begin{array}{ll}\text { Referências Bibliográficas } & 79\end{array}$ 


\title{
Lista de Símbolos
}

\author{
$\bar{S}_{h} \quad$ População de humanos suscetíveis \\ $\bar{I}_{h} \quad$ População de humanos infectados \\ $\bar{R}_{h} \quad$ População de humanos recuperados \\ $\bar{S}_{v} \quad$ População de vetores suscetíveis \\ $\bar{I}_{v} \quad$ População de vetores infectados \\ $\bar{N}_{h} \quad$ População total de humanos \\ $\bar{N}_{v} \quad$ População total de vetores \\ $S_{h} \quad$ Proporção de humanos suscetíveis \\ $I_{h} \quad$ Proporção de humanos infectados \\ $R_{h} \quad$ Proporção de humanos recuperados \\ $S_{v} \quad$ Proporção de vetores suscetíveis \\ $I_{v} \quad$ Proporção de vetores infectados \\ $b \quad$ Número médio de picadas por vetor por dia \\ $\beta_{h} \quad$ Probabilidade de transmissão de vetores para humanos \\ $\beta_{v} \quad$ Probabilidade de transmissão de humanos para vetores \\ $\lambda_{h}$ Taxa de contato efetivo de vetores para humanos $\left(b \beta_{h}\right)$ \\ $\lambda_{v} \quad$ Taxa de contato efetivo de humanos para vetores $\left(b \beta_{v}\right)$ \\ $\mu_{h} \quad$ Taxa de mortalidade natural de humanos \\ $\nu_{h}, \lambda$ Taxa de nascimentos de humanos \\ $\gamma \quad$ Taxa de crescimento da população humana \\ $k \quad$ Capacidade de carregamento humano \\ $\alpha_{h} \quad$ Taxa de mortalidade humana induzida pela doença \\ $\gamma_{h}, r$ Taxa de recuperação humana \\ $m \quad$ Número de hospedeiros alternativos \\ $N_{h} \quad$ Tamanho inicial da população humana \\ $\mu_{v} \quad$ Taxa de mortalidade natural de vetores \\ $A, D$ Taxa constante de recrutamento de vetores \\ $E_{1} \quad$ Equilíbrio livre de doença \\ $E_{2} \quad$ Equilíbrio endêmico \\ $S_{h}^{*} \quad$ Proporção de humanos suscetíveis no equilíbrio \\ $I_{h}^{*} \quad$ Proporção de humanos infectados no equilíbrio \\ $R_{h}^{*} \quad$ Proporção de humanos recuperados no equilíbrio \\ $I_{v}^{*} \quad$ Proporção de vetores infectados no equilíbrio \\ $R_{o} \quad$ Número de Reprodutibilidade Basal \\ DF Diferencial do campo de vetores $\mathrm{F}$
}




\section{Lista de Figuras}

1.1 Dados de 1990 - 2050 sobre mortes na população mundial causadas por doenças infecciosas (incluindo condições maternais e nutricionais) e doenças não infecciosas (incluindo acidentes). Em (a) são apresentadas as estimativas de mortes em 1990, 2010 e 2050. Em (b) são apresentadas 10 principais causas de morte causadas por doenças infecciosas em 2010. Os números indicados acima das barras correspondem ao número de patógenos responsáveis por causar a maior parte das mortes para cada uma das doenças. Em (c) são apresentadas as proporções das mortes causadas por doenças infecciosas e não infecciosas em países de baixa, baixa-média, média-alta e alta renda em 2010 conforme a classificação do Banco Mundial. Em (d) apresentam-se os fatores (taxa de morte, crescimento da população e envelhecimento) que afetam mudanças na porcentagem de mortes da população mundial no período de 1990 - 2010, ([Dye14]). 3

2.1 Dinâmica de propagação do dengue referente ao modelo $1 \ldots \ldots$. . . . . . . . 14

2.2 Dinâmica de propagação do dengue referente ao modelo $2 \ldots \ldots$. . . . . . . . . 29

2.3 Dinâmica de propagação do dengue referente ao modelo 3 . . . . . . . . . . . . 40

2.4 Resumo dos casos descritos no Teorema 2.3.3 . . . . . . . . . . . . . . . 48

2.5 Representação gráfica dos casos 1$)$ e 2$) \ldots \ldots \ldots \ldots \ldots$. . . . . . . . . . 52

3.1 Solução numérica do modelo 1 avaliada durante o período de 40 anos. . . . . . . . . 59

3.2 Solução numérica do modelo 3 avaliada durante o período de 40 anos. . . . . . . . . 60

3.3 Solução numérica do modelo 2 avaliada durante o período de 40 anos. . . . . . . . . . 60

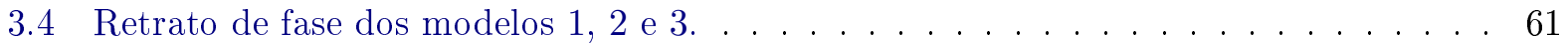

3.5 Variação do parâmetro $R_{o}$ com relação ao tempo . . . . . . . . . . . . . . 62

3.6 Variações nas soluções do modelo 1 com relação a variação de $1 \%$ na taxa de picadas do vetor. A linha tracejada corresponde a solução do modelo para o valor do parâmetro $b$, conforme definido na Tabela 3.2 . . . . . . . . . . . 66

3.7 Variações nas soluções do modelo 1 com relação a variação de $1 \%$ na taxa de morte do vetor. A linha tracejada corresponde a solução do modelo para o valor do parâmetro

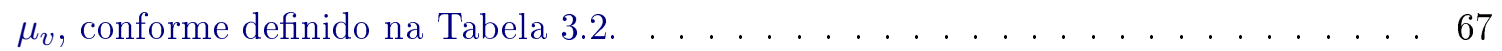

3.8 Variações nas soluções do modelo 2 com relação a variação na taxa de recuperação de humanos. A linha tracejada corresponde a solução do modelo para o valor do parâmetro $\gamma_{h}$, conforme definido na Tabela 3.2. . . . . . . . . . . . 68

3.9 Variações nas soluções do modelo 2 com relação a variação na taxa de contato efetivo de humano para vetor. A linha tracejada corresponde a solução do modelo para o valor do parâmetro $\lambda_{v}$, conforme definido na Tabela $3.2 \ldots \ldots \ldots \ldots$. . . . . 


\section{Lista de Tabelas}

3.1 Proporções endêmicas para cada modelo. . . . . . . . . . . . . . . . 58

3.2 Valores dos parâmetros de cada modelo utilizados nas simulações. . . . . . . . . . . . 58

3.3 Condições iniciais utilizadas para as simulações. . . . . . . . . . . . . . . . . . 59

3.4 Índices de sensibilidade do $R_{o}$ com relação ao valor dos parâmetros de cada modelo que estão descritos na Tabela 3.2 . . . . . . . . . . . . . . . . . 63

3.5 Índices de sensibilidade, $\Gamma_{p_{j}}^{x_{i}}=\frac{\partial x_{i}}{\partial p_{j}} \frac{p_{j}}{x_{i}}$, das varíaveis do modelo 1 no equilíbrio endêmico, com relação aos parâmetros descritos na Tabela 3.2. . . . . . . . . . . . . 65

3.6 Índices de sensibilidade, $\Gamma_{p_{j}}^{x_{i}}=\frac{\partial x_{i}}{\partial p_{j}} \frac{p_{j}}{x_{i}}$, das varíaveis do modelo 2 no equilíbrio endêmico, com relação aos parâmetros descritos na Tabela 3.2. . . . . . . . . . . . . 67

3.7 Índices de sensibilidade, $\Gamma_{p_{j}}^{x_{i}}=\frac{\partial x_{i}}{\partial p_{j}} \frac{p_{j}}{x_{i}}$, das varíaveis do modelo 3 no equilíbrio endêmico, com relação aos parâmetros descritos na Tabela 3.2. . . . . . . . . . . . . 69

3.8 Variações nas proporções do equilíbrio endêmico considerando uma variação de $1 \%$

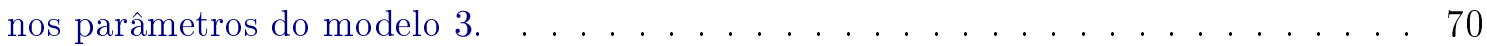




\section{Introdução}

A propagação de doenças infecciosas, tem sido ao longo da história motivo de preocupação e ameaça para saúde pública. Os problemas relacionados a propagação dessas doenças, atingem todas as esferas da sociedade, causando efeitos sociais e econômicos que prejudicam o desenvolvimento da sociedade humana. Por muitos anos, a sociedade tem lutado contra as mais diversas epidemias. Grande parte dos avanços foram registrados somente durante o século XX, quando os progressos na área médica permitiram o desenvolvimento das primeiras vacinas ([B+77], [Het00]). A varíola é um dos exemplos de doenças infecciosas que fez no mínimo 300 milhões de vítimas durante o século $X X$ e que foi totalmente erradicada após campanhas de vacinação bem sucedidas por todo o mundo. O último caso da doença foi registrado na Somália em 1977.

Enquanto a humanidade progride no estudo de novas vacinas, como é o caso do desenvolvimento de uma vacina para o vírus do dengue, ainda existe um longo caminho pela frente para erradicar completamente essas doenças. Para prevenir e controlar essas doenças de forma efetiva, é importante compreender completamente os mecanismos de transmissão e propagação dessas infecções.

O estudo da dinâmica das epidemias é uma abordagem teórica importante para investigar a dinâmica da transmissão das doenças infecciosas. Este estudo é desenvolvido através da formulação de modelos matemáticos que descrevem os mecanismos de transmissão da doença e a dinâmica dos agentes infecciosos ([Het00]). Modelos matemáticos são construídos com base na dinâmica da população, nas características dos agentes infecciosos, nos padrões de transmissão e conexões com outros fatores sociais e fisiológicos. Através de uma análise qualitativa e quantitativa, análise de sensibilidade e simulações numéricas dos modelos de propagação, o entendimento do processo de espalhamento da doença e identificação dos parâmetros mais importantes e sensíveis torna possível traçar estratégias de prevenção e controle mais eficientes ([B $\left.{ }^{+} 77\right]$, [Het00], [Het76]).

Neste trabalho, concentramos nossos esforços no estudo teórico da dinâmica de propagação do vírus do dengue. Atualmente, estima-se que cerca de 2,5 bilhões de pessoas, mais de $40 \%$ da população mundial vivem em áreas de risco. A dengue é endêmica em pelo menos 100 países na África, Américas, Mediterrâneo Oriental, Sudeste da Ásia e Pacífico Ocidental. Recentes estimativas da Organização Mundial da Saúde (OMS) apontam que cerca de 390 milhões de infecções ocorrem anualmente, incluindo 500.000 casos de dengue hemorrágica e 22.000 mortes, principalmente entre crianças. As regiões das Américas, Sudeste da Ásia e Pacífico Ocidental são as mais afetadas. Nas três regiões houveram mais de 1,2 milhões de casos de dengue em 2008 e mais de 3 milhões em 2013, apresentando uma tendência de crescimento ao longo dos anos ([Org]).

O vírus do dengue é transmitido por vetores e tem se espalhado rapidamente ao redor do mundo. Na Europa, em 2010, foram registrados casos de dengue autóctones na França e na Croácia, e ainda foram detectados três casos importados em outros três países do continente. Em 2012, um surto na ilha de Madeira, em Portugal, resultou em mais de dois mil casos. Em 2013, ocorreram casos nos 
Estados Unidos e na China. Em 2014, tendências indicam aumento no número de casos nas Ilhas Cook, Fiji, Malásia e Vanuatu. A doença também foi registrada no Japão após um intervalo de mais de 70 anos. No Brasil, a dengue está espalhada em praticamente por todo território nacional. Segundo boletins epidemiológicos, o país registrou cerca de 1.054 .127 prováveis casos de dengue durante o período de janeiro a abril de 2016. A região sudeste responde por $59 \%$ destes casos ([Org], [dS]).

A infecção pelo vírus do dengue é causado por 4 sorotipos distintos: DEN-1, DEN-2, DEN3, DEN-4 que pertencem ao gênero Flavivirus, da família Flaviviridae. Genótipos distintos foram identificados em cada sorotipo o que caracteriza a grande variabilidade genética do vírus ([Org]).

O mecanismo de contração da doença é pela picada da fêmea do mosquito infectada, sendo o mosquito Aedes aegypti o principal vetor de transmissão da doença seguido pelo Aedes albopyctus. Uma vez picados pela fêmea do mosquito Aedes, os seres humanos tornam-se os portadores e replicadores do vírus, tornando-se ainda uma nova fonte de vírus para outros mosquitos não infectados ([Org]).

A dengue manifesta-se predominantemente em zonas tropicais e subtropicais, cujas condições climáticas favorecem o desenvolvimento dos ovos e larvas do mosquito. Muitos fatores têm sido combinados para formar condições adequadas ao desenvolvimento da epidemia, dentre eles estão fatores ambientais e socioeconômicos incluindo o crescimento desordenado da população, a migração de pessoas da zona rural para a zona urbana, condições de infraestrutura básica inadequadas (coleta seletiva de lixo, condições de saneamento básico, etc), além dos aspectos geográficos de cada região.

No Capítulo 1, baseados em [B $\left.{ }^{+} 77\right]$, desenvolvemos inicialmente uma revisão teórica acerca da evolução das ideias em epidemiologia matemática, buscando traçar um paralelo entre o surgimento das doenças e como as ideias matemáticas acompanharam e adaptaram essas informações na evolução dos modelos. Em seguida, estudamos como o conceito de Número de Reprodutibilidade Basal $\left(R_{o}\right)$ foi introduzido na epidemiologia matemática, bem como estruturou-se a definição deste parâmetro através da metodologia da construção do Operador de Próxima Geração $\left(\left[\mathrm{B}^{+} 75\right]\right)$. No contexto epidemiológico, o parâmetro $R_{o}$ mede o número de casos secundários causados por um único indivíduo infectado ao ser introduzido em uma população completamente suscetível durante o seu período infeccioso.

No Capítulo 2, baseados nos trabalhos [EV98], [EV99] e [LJ14], desenvolvemos uma análise teórica qualitativa de um modelo de propagação de dengue, considerando diferentes hipóteses sobre a população humana total, $N_{h}(t)$. Nossa escolha pelas hipóteses sobre o crescimento da população humana deu-se por dois motivos: $i$ ) pois essas variações são amplamente discutidas na literatura para os mais diversos modelos de propagação e $i i$ ) porque queremos avaliar o impacto destas hipóteses no cálculo do parâmetro $R_{o}$ e equilíbrio endêmico. O modelo matemático estudado corresponde a um modelo compartimental do tipo SIR (Suscetível-Infectado-Recuperado), com acoplamento da dinâmica do vetor. Neste capítulo, são apresentadas ferramentas discutidas amplamente na literatura usadas para a construção de uma base teórica para o estudo qualitativo de modelos epidemiológicos. Objetivamos apresentar alguns resultados importantes da teoria qualitativa de Equações Diferenciais Ordinárias, teoria de Sistemas Competitivos e teoria de Bifurcações, aplicados no estudo da estabilidade local e global dos pontos de equilíbrio associados ao modelo matemático de propagação de dengue ([LM95]).

No Capítulo 3, fazemos um estudo numérico das soluções dos modelos de propagação analisados 
no capítulo 2. A solução numérica dos modelos matemáticos foi obtida com o método Rosenbrock de ordem 3 e 4 estágios, implementado em linguagem Fortran90 ([Sar14]). Os resultados foram apresentados em termos das proporções de indivíduos em cada uma das classes e foram avaliados no período de 40 anos. Este período foi definido de forma que fosse possível verificarmos numericamente os resultados teóricos apresentados no Capítulo 2. A partir de uma base de parâmetros obtidas da literatura, foram obtidas diferentes estimativas para o valor do parâmetro $R_{o}$, o qual caracteriza o potencial inicial de transmissão da doença. Além de apresentarmos a solução numérica, foi realizada uma análise de sensibilidade com o intuito de determinar a importância relativa dos parâmetros do modelo na transmissão da doença, através da sensibilidade do limiar $R_{o}$, e prevalência da doença, através da análise de sensibilidade do equilíbrio endêmico ([CHC08]).

Finalmente, apresentamos uma conclusão sobre os resultados obtidos e o Apêndice. No Apêndice A, apresentamos algumas definições e resultados necessários para o estudo da estabilidade dos pontos de equilíbrio do modelo de propagação de dengue descrito no Capítulo 2. 


\section{Capítulo 1}

\section{Fenômenos epidemiológicos e a modelagem matemática}

Epidemias de doenças infecciosas têm sido documentadas ao longo da história. Desde a Grécia antiga e antigo Egito tem-se relatos de epidemias de varíola, lepra, tuberculose e difteria. As condições de higiene inadequadas e os escassos recursos da medicina da época, fizeram com que a eficiência na propagação e a letalidade dessas doenças tivessem consequências catastróficas para a raça humana atingindo magnitudes semelhantes a das guerras. Motivados pelas aplicações da modelagem matemática no estudo de doenças infecciosas, desenvolvemos este capítulo com o intuito de expor o progresso das ideias em epidemiologia matemática, bem como sua a sua fundamentação teórica. Apresentamos o processo de construção e formalização matemática do conceito do número de reprodutibilidade basal, parâmetro que mede o número de casos secundários causados por um único indivíduo infectado ao ser introduzido numa população totalmente suscetível.

\subsection{Motivação}

Em 1918, após o final da primeira guerra mundial, que matou pelo menos 37 milhões de pessoas, o mundo foi abalado pela gripe espanhola. Acredita-se que a pandemia de gripe tenha sido responsável por infectar cerca de 500 milhões de pessoas ao redor do mundo, incluindo ilhas remotas do Pacífico e Ártico, resultando em cerca de 50 a 100 milhões de mortes. Durante o século 14, de 1347 a 1353, a peste negra foi responsável por dizimar cerca de $25 \%$ da população europeia que na época estimava-se em 100 milhões de pessoas $\left(\left[\mathrm{B}^{+} 77\right]\right)$.

Um pouco mais tarde, tendo sido importada da Europa pelos colonizadores em 1952, a varíola foi responsável pela morte de praticamente metade da população asteca. Já na América do Sul, em (1648), dados estimam que 280 mil pessoas morreram em função da febre amarela. A malária atualmente é endêmica nas regiões equatoriais, em regiões da América, Ásia e grande parte da África. Segundo a ONU cerca de 350 milhões de pessoas vivem em áreas endêmicas ([B $\left.\left.{ }^{+} 77\right]\right)$.

A grande praga de Londres cuja ocorrência é datada por volta de 1665 foi uma epidemia em escala menor se comparada com a peste negra que atingiu a Europa (1347 - 1353), entretanto, devido à eficiente capacidade de propagação da doença comprovada pelo acelerado crescimento do número de casos, a doença passou a ser monitorada semanalmente ([B $\left.\left.{ }^{+} 77\right]\right)$. Durante essa epidemia estimou-se que o número de mortes causadas pela doença em determinada semana foi 37 vezes 
maior que o número de nascimentos da mesma semana.

Após a descoberta de que eram os roedores os reservatórios da doença, a eliminação dos mesmos, melhores condições de higiene e a resistência genética das populações contribuíram para o declínio dessas epidemias na Europa $\left(\left[\mathrm{B}^{+} 77\right]\right)$.

A Figura 1.1, obtida em ([Dye14]) apresenta a distribuição das causas de mortes da população mundial causadas por doenças infecciosas (incluindo causas nutricionais e maternais) e não infecciosas (incluindo acidentes) para os anos de 1990, 2010 e 2050. Em a) são apresentadas as estimativas de mortes causadas por doenças infecciosas e não infecciosas para os anos de 1990, 2010 e 2050. É possível observarmos um decréscimo das mortes causadas por doenças infecciosas, em contrapartida, observamos uma aumento considerável do número de mortes causadas por doenças não infecciosas, principalmente de 2010 para 2050 quando o número de mortes de doenças causadas por doenças não infecciosas praticamente dobrou. Em $b$ ) é apresentada a distribuição das causas de mortes dentro da classe das doenças infecciosas. Para o ano de 2010, das mortes causadas por doenças infecciosas destacam-se as doenças respiratórias que correspondem a mais de 3 milhões de mortes.

Em c), dados de 2010 apresentam como as causas de mortes por doenças infecciosas e não infecciosas estão relacionadas às condições de renda de cada região. Predominantemente temos um número maior de mortes causadas por doenças não infecciosas em regiões cuja renda varia de de média a alta. Por outro lado, em regiões de baixa renda temos como maior causa de morte as doenças infecciosas. Estas estatísticas para regiões mais pobres vinculam-se diretamente às condições sanitárias que por sua vez colaboram com o desenvolvimento de patógenos. Por fim em $d$ ) são apresentados os fatores que contribuem com a redução ou aumento das causas de mortes causadas por doenças infecciosas e não infecciosas. Como era esperado, o aumento no crescimento da população favorece a transmissão e propagação de doenças infecciosas. A taxa de morte per capita é inversamente proporcional ao número de mortes causadas pelas doenças infecciosas. Conforme temos um aumento na taxa de morte per capita vamos ter uma diminuição na porcentagem de mortes causadas por doenças infecciosas. Essa observação reflete a relação entre o aumento no número de mortes causadas por doenças infecciosas e o aumento na população.

Neste contexto, a modelagem matemática aplicada a investigação da dinâmica da propagação de doenças infecciosas tem demonstrado ser uma ferramenta poderosa para o entendimento dessas doenças. Desde os primeiros modelos em meados do século XVII, contribuições significativas foram obtidas para a população em nível local ou global, pois o conhecimento adquirido durante anos sobre as doenças infecciosas foi incorporado aos modelos, aprimorando assim suas contribuições e evidenciando a relevância e aplicabilidade desta área de pesquisa. 

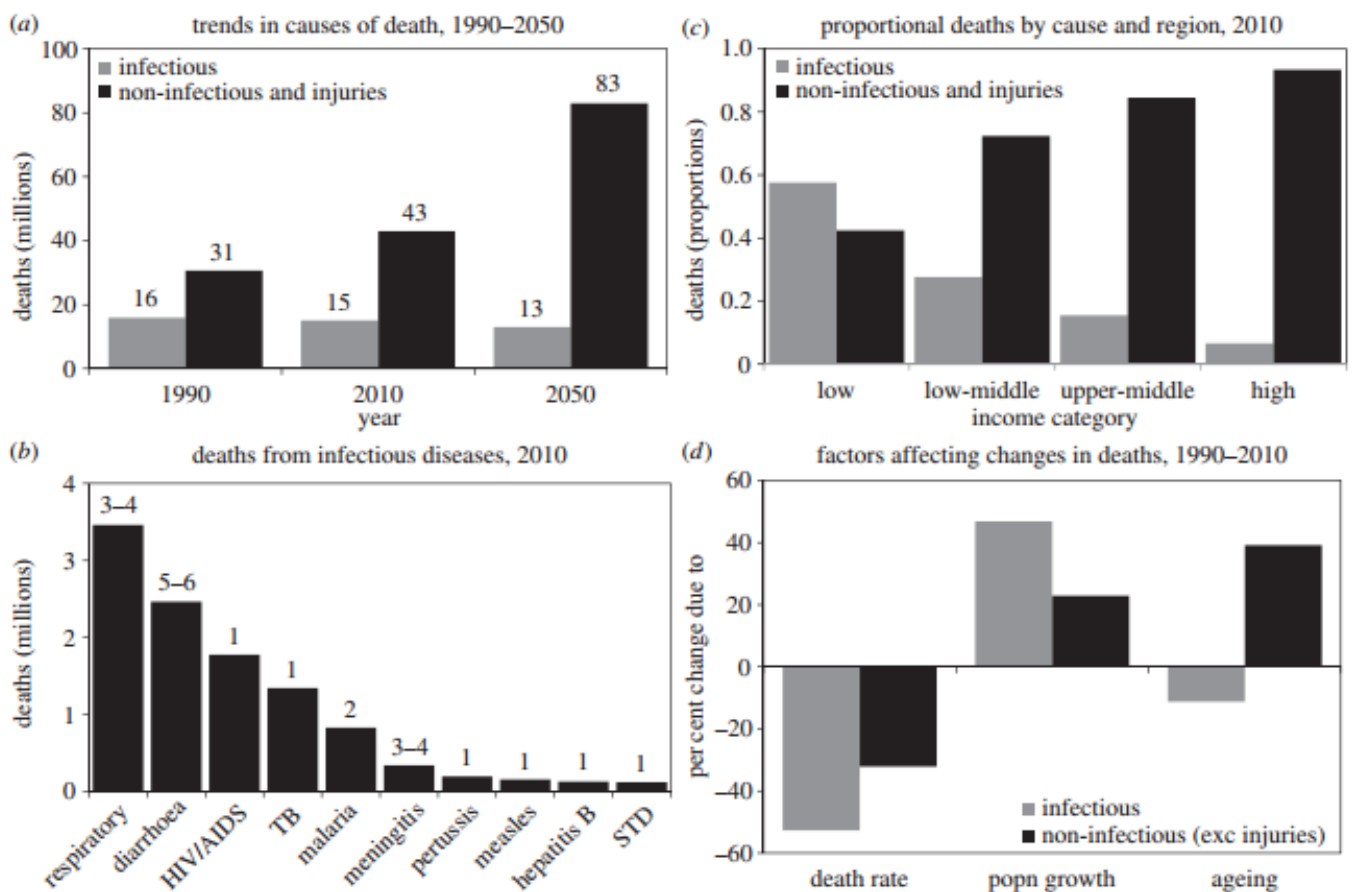

Figura 1.1: Dados de 1990 - 2050 sobre mortes na população mundial causadas por doenças infecciosas (incluindo condições maternais e nutricionais) e doenças não infecciosas (incluindo acidentes). Em (a) são apresentadas as estimativas de mortes em 1990, 2010 e 2050. Em (b) são apresentadas 10 principais causas de morte causadas por doenças infecciosas em 2010. Os números indicados acima das barras correspondem ao número de patógenos responsáveis por causar a maior parte das mortes para cada uma das doenças. Em (c) são apresentadas as proporções das mortes causadas por doenças infecciosas e não infecciosas em países de baixa, baixa-média, média-alta e alta renda em 2010 conforme a classificação do Banco Mundial. Em (d) apresentam-se os fatores (taxa de morte, crescimento da população e envelhecimento) que afetam mudanças na porcentagem de mortes da população mundial no periodo de 1990 - 2010, ([Dye14]).

\subsection{Evolução dos modelos epidemiológicos}

O estudo de modelos epidemiológicos surgiu por volta de 1760 com um modelo matemático para inoculação da varíola proposto pelo matemático Daniel Bernoulli, cujo objetivo era estimar o ganho na expectativa de vida de um indivíduo ao nascer, se a varíola fosse eliminada como causa de morte ([DH02], [Het00]).

Em 1906, Hamer evidenciou o que foi considerada uma das maiores contribuições teóricas em epidemiologia. Ele foi um dos pioneiros a assumir que o número de novos casos de indíviduos infectados eram causados pelo produto entre as densidades de indivíduos suscetíveis e infectados ([Het00]). Esta hipótese matemática formulada por Hamer é a base de todas as teorias determinísticas subsequentes. Esta hipóstese ficou conhecida como o princípio da ação de massas, pois compara-se a uma reação química $\left(\left[\mathrm{B}^{+} 75\right]\right)$. Usando estas ideias, Hamer pode deduzir a existência de recorrências periódicas da doença. Uma extensão dessas ideias foi desenvolvida posteriormente por Soper, em 1929. Por volta de 1908, Ross trabalhou com um modelo matemático mais estruturado, tomando um conjunto básico de parâmetros que descreviam aspectos relacionados a transmissão da malária $\left(\left[\mathrm{B}^{+75}\right]\right)$.

No entanto, foi em 1927 que estudos matemáticos mais elaborados foram desenvolvidos por Kermack e McKendrick. Eles propuseram e resolveram um dos teoremas mais importantes em epidemiologia, o fenômeno limiar, o qual evidenciava que para se ter um surto epidêmico a densidade 
de indíviduos suscetíveis deveria exceder um determinado valor crítico, denominado limiar ([Het00], [KM27], [And91]). Desde então, os estudos em epidemiologia matemática têm avançado consideravelmente. Algumas das leituras clássicas sobre a evolução dos primeiros conceitos em epidemiologia e sobre o desenvolvimento das ideias matemáticas associadas aos modelos epidemiológicos foram publicadas por Bailey (1975) e Becker (1979), Dietz (1974), Anderson e May (1982) ([B $\left.\left.{ }^{+} 75\right]\right)$.

Em geral, modelos matemáticos clássicos neste estudo são os modelos determinísticos e estocáticos. Modelos determinísticos são usados em geral para descrever a dinâmica de grandes populações. Nesses modelos, os parâmetros de entrada (como por exemplo, taxa de morte da doença ou taxa de recuperação) são fixados e portanto as predições do modelo ao longo do tempo são de certa forma predeterminadas. A maior parte dos modelos determinísticos são compartimentais e a representação matemática desses compartimentos é feita por meio de equações diferenciais ordinárias.

Dentre os modelos determinísticos, as estruturas mais usadas são: MSEIR, MSEIRS, SEIR, SEIRS, SIR, SIRS, SEI, SEIS, SI, SIS, onde o compartimento M representa a classe dos indivíduos que adquiriram algum tipo imunidade através da placenta de suas mães infectadas, este tipo de transmissão é conhecida como transmissão vertical. O compartimento S representa a classe dos indivíduos suscetíveis, E são os indíviduos latentes, ou seja, estão infectados mas ainda não transmitem a doença, I são os indivíduos infectados e R corresponde ao compartimento dos indivíduos recuperados ou removidos, seja por adquirirem imunidade ou por morte pela doença. Podemos observar na literatura a ampla aplicação destes modelos nas mais diversas doenças: dengue, cólera, malária, tuberculose, HIV (ver [Nis06],[CCS02], [KR08], [Het00], [EV99], [CS78], [B $\left.{ }^{+} 75\right]$, [CCS04], [AM91]).

McKendrick, em 1926, foi um dos primeiros a publicar trabalhos que envolvessem tratamento estócástico aos modelos epidêmicos $\left(\left[\mathrm{B}^{+} 75\right]\right)$. Modelos estocásticos permitem que o número de indivíduos que se deslocam entre os compartimentos varie aleatoriamente. Essa característica dos modelos estocásticos oferece facilidades na inclusão de heterogeneidades da doença aos modelos. Matematicamente, a representação dos compartimentos em modelos estocásticos, é feita por meio de equações diferenciais parciais. Contudo, estes modelos tendem a ser mais utilizados quando o estudo envolve pequenas populações, pois quando o tamanho das populações for grande é possível mostrar que as soluções fornecidas pelo modelo estocástico convergem para as soluções do modelo determinístico $\left(\left[\mathrm{B}^{+} 75\right]\right)$.

Uma vez que, os modelos matemáticos tenham essencialmente a mesma base, a inserção de diferentes hipóteses torna-os flexíveis para o estudo de qualquer doença. Dependendo da complexidade destes modelos, métodos numéricos são mais adequados para a obtenção das soluções, posto que a obtenção da solução analítica não é possível. Entretanto, o que se tem observado é que o comportamento qualitativo obtido pela resolução desses modelos, em alguns casos não condiz com o comportamento dos dados reais.

Um exemplo disso, foi a projeção feita para o números de casos de novos casos de ebola em Montserrado, na Libéria. A modelagem foi desenvolvida a partir da construção de um modelo SEIR, cujos parâmetros foram estimados e corrigidos através de simulações do método Monte Carlo. O modelo estimou que ocorreriam mais de 170 mil casos de outubro de 2014 a dezembro do mesmo ano, que não se confirmaram em dezembro ([LMAM $\left.\left.{ }^{+} 14\right]\right)$.

A correção e aperfeiçoamento dos modelos exige um estudo interdisciplinar. Novos aspectos instrínsecos ou extrínsecos da doenças são introduzidos aos modelos matemáticos na forma de 
parâmetros e variáveis permitindo a formulação de um modelo matemático tão complexo quanto se queira, inclusive o acoplamento de duas dinâmicas, como é o caso de doenças transmitidas por vetores ou coexistência de vários sorotipos.

No caso de doenças cuja transmissão é direta e com alta letalidade, tem-se em geral, dados mais consistentes, pois existe uma maior preocupação dos órgãos da saúde em evitar que essas doenças se propaguem. No entanto, mesmo com um monitoramento detalhado da doença, mensurar parâmetros como, por exemplo, número médio de contatos adequados entre indivíduos suscetíveis e infectados não é uma tarefa simples.

Para doenças transmitidas indiretamente, a situação é ainda mais complicada. A dengue em particular é um exemplo crítico. Apesar de existirem dados sobre as notificações em todo território nacional, acompanhado pelo Ministério da Saúde, percebe-se variações no comportamento dos surtos epidêmicos de uma região para outra e inclusive dentro de uma mesma região. Neste sentido, muitas discussões o envolvimento de outros fatores, não disponíveis nestes bancos, como precipitação, clima, condições socioeconômicas da população e condições imunológicas dos pacientes com relação a outras doenças ([MF98], [dS10], [WLG+ 09], [HWSW99]).

$\mathrm{Na}$ abordagem epidemiológica, até pouco antes do final do século XX, não haviam muitas discussões acerca do estudo de outras funções que representassem a transmissão de doenças exceto, o princípio da ação de massas. Estudos recentes, sugerem uma reavaliação nos padrões de contato das doenças, propondo o uso de outras funções de transmissão como, funções de transmissão dependente das densidades de indivíduos e funções de transmissão não lineares. Destacamos aqui alguns artigos que tentam esclarecer controvérsias acerca de diferentes cenários epidemológicos através do estudo de diferentes funções de transmissão (ver [CLL13], [Nov08], [HLVDD89],[LLI86], [LHL87], [WLRVdD06], [KM05], [HVdD91]).

Enquanto que para as doenças emergentes vacinas ainda não foram desenvolvidas, estratégias de controle com relação a transmissão são medidas de contenção que devem ser tomadas, seja no caso de doenças transmitidas direta ou indiretamente. Nesta perspectiva, a influência da mobilidade humana na propagação de doenças tem sido estudada através da construção de redes de contato (consultar os trabalhos [DPE13], [WET ${ }^{+}$12], [Kee05], [SD95], [KDVH10], [BDG09], [Ari09], [SFM+13], [ISS13], [AK09]).

Considerando que, todos os modelos são aproximações e que portanto a questão principal consiste em obtermos um modelo que não perde a essência do problema real teremos um acréscimo de imprecisões quanto mais elementos vão sendo incorporados. Sabe-se que em epidemiologia é muito comum que a maior parte dos parâmetros não tenha aferição direta, isso implica no uso de técnicas para estimar esses parâmetros. Em geral são usadas técnicas estatísticas como: método Cadeia de Markov Monte Carlo e hipercubo latino por exemplo ([GRS96], [BD94]).

Desde a última década, a velocidade computacional tem melhorado muito e com isso, resolver numericamente modelos matemáticos sofisticados não tem sido um problema. O uso desta ferramenta, além de fornecer as soluções dos modelos, nos permite a exploração de diferentes cenários, o que em termos de doenças epidêmicas, muito além de tentar predizer epidemias, podem auxiliar na criação de estratégias de controle e prevenção. 


\subsection{Aspectos teóricos dos modelos de propagação de epidemias}

Essencialmente, espera-se que os modelos matemáticos satisfaçam quatro atributos: robustez, flexibilidade, precisão e capacidade preditiva. A robustez caracteriza-se pela habilidade do modelo em de reproduzir os dados observados e capturar a dinâmica da doença a longo prazo, ou seja, a robustez é responsável por capturar tanto o comportamento qualitativo quanto quantitativo da doença ([KR08]).

A flexibilidade do modelo está associada a capacidade de adaptação do mesmo a outros fenômenos similares e também a rápida assimilação de novas observações. A precisão por sua vez, é definida no sentido de convergência numérica das soluções do modelo. No caso dos modelos epidemiológicos espera-se que a solução convirja assintoticamente para os equilíbrios com uma precisão pré-fixada. A capacidade preditiva consiste na obtenção de estimativas futuras.

Ao fazer um estudo qualitativo, podemos identificar propriedades com relação a dinâmica do comportamento das soluções, como estabilidade e convergência. O estudo do comportamento quantitativo desses modelos em geral é realizado por meio de simulações numéricas pode fornecer estimativas preditivas que serão avaliadas por órgãos de saúde pública no desenvolvimento de estratégias de controle.

A abordagem numérica como ferramenta de apoio à modelagem, permite o estudo de diferentes cenários, a validação de conjecturas qualitativas ou quantitativas sobre a dinâmica, a estimativa de parâmetros bem como avaliar a sensibilidade do modelo em relação às condições iniciais e parâmetros.

\subsection{Parâmetros epidêmicos}

\subsubsection{Força de infecção, $\lambda$}

Em epidemiologia clássica, a força de infecção é definida como densidade de incidência, ou seja, corresponde ao número de casos novos por unidade de tempo divido pela população total. Este parâmetro pode ser obtido a partir dos dados epidemiológicos considerando a hipótese de que no estágio inicial da epidemia o número de indivíduos infectados cresce aproximadamente a uma taxa constante, essa taxa refere-se a taxa de crescimento da epidemia. Em outras palavras, o número de infectados podem ser escrito como:

$$
I(t) \approx I(0) e^{\lambda t}
$$

onde $I(0)$ corresponde ao número de indíviduos infectados no tempo zero.

Uma das formas de fazer o cálculo da força de infecção é ajustar uma reta ao número de casos acumulados. O cálculo da força de infecção a partir do uso dos dados acumulados é razoável, pois esse método suaviza os dados num estágio crítico da doença, o estágio inicial, onde devemos obter o valor de $\lambda$ e onde podem ocorrer as maiores flutuações ([VW10]). Outros métodos estatísticos envolvendo técnicas como o Método da Máxima Verossimilhança são usados para obter a força de infecção em idades específicas baseados em dados sorológicos estratificados por idade ([GA85]). 


\subsubsection{Número de Reprodutibilidade Basal, $R_{o}$}

Epidemiologicamente, o número de reprodutibilidade basal é conhecido como $R_{o}$. Este parâmetro corresponde ao número médio de infectados secundários causados por um único indivíduo infectado em uma população totalmente suscetível enquanto ele for infectante. Mais precisamente, este parâmetro caracteriza a habilidade de um organismo infeccioso invadir uma população totalmente suscetível e estabelecer-se nela durante o período em que estiver infeccioso. Se $R_{o}>1$ a doença conseguirá invadir uma população e estabelecer-se nela. Se $R_{o} \leq 1$ a doença não conseguirá se estabelecer na população e desaperarecerá naturalmente.

Para doenças de transmissão direta e com dependência etária, o valor do $R_{o}$ pode ser obtido através da força de infecção. Analiticamente e epidemiologicamente esses dois limiares relacionamse. Considerando, $L$ como sendo a expectativa média de vida da população, $R_{o}$ por ser escrito em função de $\lambda$ como sendo: $R_{o}=1+\lambda L$. A derivação desta expressão e maiores discussões acerca dessa formulação podem ser encontradas em [AM91] e [Het00].

Dependendo da complexidade do modelo matemático pode-se obter o valor de $R_{o}$ diretamente das equações do modelo. Considere o modelo SIR (Suscetível - Infectado - Recuperado) proposto por Kermack e McKendrick, em [Het00], com dinâmica vital e governado pelas seguintes equações:

$$
\begin{array}{rlrl}
\frac{d S}{d t} & =\mu N-\beta S \frac{I}{N}-\mu S & S(0) & =S_{o} \geq 0 \\
\frac{d I}{d t} & =\beta S \frac{I}{N}-(\mu+\gamma) I & I(0) & =I_{o} \geq 0 \\
\frac{d R}{d t} & =\gamma I-\mu R & R(0) & =R_{o} \geq 0
\end{array}
$$

onde $N=S+I+R$ é constante, $\mu$ corresponde a taxa de nascimento e morte natural, $\gamma$ é a taxa per capita de recuperação da infecção e $\beta$ corresponde ao número de contatos adequados entre suscetíveis e infectados. Nesse sentido, contatos adequados significam contatos que resultem em infecção. Visto que, $\beta$ corresponde ao número médio de suscetíveis infectados por um único indivíduo infectado por unidade de tempo e $\frac{1}{\mu+\gamma}$ é o tamanho do período infeccioso temos portanto, $R_{o}=\frac{\beta}{\mu+\gamma}([\mathrm{KR} 08])$.

O modelo acima é bastante simples, e neste caso, a dedução do valor de $R_{o}$ é direta. Para modelos mais complexos, a expressão deste parâmetro torna-se complicada de ser obtida diretamente das equações do modelo. Uma das alternativas para contornar este problema é obter o Operados de Próxima Geração. Diekmann, Heesterbeek e Metz, em [DHR09], propuseram uma metodologia para a construção do Operador de Próxima Geração, $(O P G)$, para modelos compartimentais. Neste caso o valor de $R_{o}$ é definido como sendo o raio espectral deste operador.

Biologicamente, o parâmetro $R_{o}$ está associado a transmissão da infecção como se fosse um processo demográfico, no sentido que os nascimentos não seriam nascimentos demográficos mas sim um nascimento epidemiológico causado pela infecção através de alguma forma de transmissão. Naturalmente, somos capazes de entender e visualizar este processo de infecção em termos de gerações consecutivas de indivíduos infectados o que nos possibilita fazer uma analogia com as gerações demográficas ([DHM90], [HD96]). As gerações subsequentes de indivíduos infectados, crescem em tamanho indicando uma população cada vez maior, no sentido epidemiológico indicando que teremos uma epidemia e o fator de crescimento por geração indica o potencial de crescimento dessa 
infecção. Matematicamente, esse fator de crescimento é a caracterização do $R_{0}$.

\subsubsection{Formalização do conceito de $R_{o}$ baseado na teoria demográfica}

O valor crítico $R_{o}$ não surgiu inicialmente em epidemiologia, mas em demografia. Richard Böckh foi um dos primeiros a mencionar o conceito em 1884 como "reprodução total" o qual fazia referência ao número de fêmeas nascidas de uma única fêmea durante o seu ciclo de vida reprodutivo. Dublin e Lotka (1925) e Kuczynski (1928) formalizaram o cálculo e introduziram a notação de $R_{o}$ no sentido demográfico. Neste contexto, o termo usado para $R_{o}$ era de taxa líquida de reprodução por geração ([HD96]).

Conforme esta definição, o valor crítico de $R_{o}$ ficou definido como:

$$
R_{o}=\int_{0}^{\infty} b(a) F(a) d a
$$

onde $F(a)=e^{-\int_{0}^{a} \mu(\alpha) d \alpha}$ corresponde a função sobrevivência (observe que a função sobrevivência está relacionada com a mortalidade na idade específica, $\mu(a))$, isto é, corresponde a probabilidade de um indivíduo recém nascido sobreviver pelo menos até a idade $a$ e $b(a)$ corresponde ao número médio de descendentes que um indivíduo produzirá por unidade de tempo até a idade $a$.

Portanto, se $R_{o}>1$, ou seja, se em média cada fêmea contribuir com mais de uma fêmea por geração a população irá crescer. Este crescimento populacional é então caracterizado pelo crescimento de gerações subsequentes de indivíduos. A partir dessa conclusão, este conceito limiar passou a ser associado ao conceito epidemiológico.

Seja $n(t, a)$ o número de indivíduos em tempo t distribuídos no intervalo $\left[a_{1}, a_{2}\right]$ dado pela integral $\int_{a_{1}}^{a_{2}} n(t, a) d a$. A equação diferencial parcial que representa o crecimento da população distribuída por idade é dada por:

$$
\frac{\partial n}{\partial t}(t, a)+\frac{\partial n}{\partial a}(t, a)=-\mu(a) n(t, a),
$$

onde $\mu(a)$ é a taxa de morte específica na idade $a$.

Sendo $b(a)$ a fertilidade por pessoa em idade $a$, temos que os nascimentos em tempo $t$ são dados por:

$$
B(t)=n(t, 0)=\int_{0}^{\infty} b(a) n(t, a) d a,
$$

cuja distribuição inicial de idade é dada por: $n(0, a)=n_{o}(a)$ com $n_{o}(0)=B(0)$.

Este modelo demográfico foi usado por Lotka, em 1922, na modelagem de populações, por McKendrick, em 1926, no estudo de modelos epidêmicos e por von Foerster no estudo da proliferação celular ([Het00]).

Resolvendo a equação (1.4) obtemos:

$$
n(t, a)= \begin{cases}B(t-a) e^{-\int_{0}^{a} \mu(v) d v} & \text { para } t \geq a \\ u_{o}(a-t) e^{-\int_{a-t}^{a} \mu(v) d v} & \text { para } t<a .\end{cases}
$$

Substituindo o limite da integral na expressão (1.5) em $a=t$ obteremos:

$$
B(t)=n(t, 0)=\int_{0}^{t} b(a) B(t-a) e^{-\int_{0}^{a} \mu(v) d v} d a+\int_{t}^{\infty} b(a) n_{o}(a) e^{-\int_{a-t}^{a} \mu(v) d v} d a
$$


Esta equação com um núcleo $K(a)$ na primeira integral e $g(t)$ na segunda integral torna-se a equação de renovação:

$$
B(t)=n(t, 0)=\int_{0}^{t} K(a) B(t-a) d a+g(t) .
$$

Analisando esta equação obtemos que a distribuição de idades se aproxima do estado de equilíbrio $A(a)$ e que o tamanho da população é aproximado exponencialmente por um crescimento ou decrescimento da forma $e^{r t}$. Portanto, ao analisar o comportamento assintótico da distribuição $A(a)$ (ver detalhes em [Het00], [HD96]), obteremos a equação característica de Lotka:

$$
1=\int_{0}^{\infty} b(a) F(a) e^{-r a} d a,
$$

onde $r$ é a taxa de crescimento intrínseco da população.

Se o número de reprodução da população é:

$$
R_{\text {pop }}=\int_{0}^{\infty} b(a) F(a) d a,
$$

então temos a seguinte observação:

Observação 1.4.1. Se $R_{o}$ é definido como na equação (1.3), de acordo com (1.8) temos:

i. $R_{o}>1$ se, e somente se $r>0$;

ii. $R_{o}=1$ se, e somente se $r=0$;

iii. $R_{o}<1$ se, e somente se $r<0$.

Até o momento descrevemos duas formas de caracterização para o crescimento e decrescimento de populações. A primeira baseou-se em gerações discretas, calculando a próxima geração de fêmeas a partir de uma outra fêmea. A segunda forma baseia-se no crescimento populacional em tempo real. Uma desvantagem do segundo método é que, pelo fato da caracterização da taxa de crescimento ser dada implicitamente, o cálculo do parâmetro $r$, por conseguinte, tem de ser feito numericamente.

Nosso próximo passo será caracterizar e contextualizar a definição de $R_{o}$ na teoria epidemica, relacionando este limiar ao parâmetro $\lambda$ (força de infecção), observando que, ambas as aproximações ocorrem quando caracterizamos crescimento ou decrescimento de subpopulações infectadas por uma dada infecção.

\subsubsection{Formalização do conceito de $R_{o}$ a partir de um modelo epidêmico}

Em epidemiologia o conceito de $R_{o}$ foi mencionado inicialmente por Ronald Ross, em 1911, em seu estudo sobre a malária e em seguida definido por Kermack e McKendrick através do teorema do limiar. Ao descrever este limiar para modelos epidêmicos, Kermack e McKendrick fizeram as seguintes hipóteses ([DHM90]):

i. Uma única infecção desencadeia um processo autônomo dentro do hospedeiro;

ii. A doença resulta de imunidade completa ou morte;

iii. Os contatos ocorrem de acordo com a lei da ação de massas; 
iv. Todos os individuos são igualmente suscetíveis;

v. A população é fechada, ou seja, na escala de tempo da transmissão da doença a entrada de novos suscetíveis é negligenciada;

vi. O tamanho da população é grande para garantir uma descrição determinística.

Seja $S(t)$ a densidade de suscetíveis na população em tempo $t$. A hipótese $i$. permite uma representação da idade para o estado de infecção (infectividade) de um indivíduo infectado, ou seja, o tempo decorrido desde a infecção denomina-se idade da infecção. Sob as hipóteses acima a equação integral para a variação de suscetíveis é dada por:

$$
S^{\prime}(t)=S(t) \int_{0}^{\infty} A(\tau) S^{\prime}(t-\tau) d \tau
$$

onde $A(\tau)$ corresponde a infectividade esperada de um indivíduo com idade de infecção $\tau$. $A(\tau)$ pode ser interpretada como uma função de reprodução onde cada descendente produzido representa uma nova infecção causada.

Na equação $(1.10),-S^{\prime}(t)$ representa a incidência $i(t, 0)$, que corresponde a densidade de novos infectados por unidade de tempo, avaliados no tempo $t$ :

$$
-S^{\prime}(t-\tau)=i(t, \tau)
$$

isso nos dá a incidência de infectados em tempo $t$ que foram infectados em tempo $\tau$ ([DHM90]).

Substituindo (1.11) na equação (1.10) obtemos o número de indivíduos infectados em $t-\tau$ unidades de tempo:

$$
\begin{gathered}
\frac{\partial i}{\partial t}+\frac{\partial i}{\partial \tau}=0, \\
i(t, 0)=S(t) \Lambda(t, \tau)
\end{gathered}
$$

cujo, $\Lambda(t, \tau):=\int_{0}^{\infty} A(\tau) i(t, \tau) d \tau$ correponde a força de infecção que mede a probabilidade per capita por unidade de tempo para tornar-se infectado ([HD96]).

Para caracterizarmos o crescimento ou decrescimento de uma população infectiva procederemos como anteriormente. Substituindo $S(t)$ na equação (1.10) pela constante $S_{o}$, onde $S_{o}$ corresponde a densidade inicial de indivíduos suscetíveis $\left(S_{o}\right.$ corresponde ao estado de equilíbrio demográfico da população na ausência da doença), obteremos

$$
S^{\prime}(t)=S_{o} \int_{0}^{\infty} A(\tau) S^{\prime}(t-\tau) d \tau
$$

Conforme Heesterbeek e Dietz [HD96], é possível mostrar que a equação (1.13) tem soluções da forma $-S^{\prime}(t)=i(t, 0)=c e^{\lambda t} \operatorname{com} \lambda>0$ se, e somente se $R_{o}>1$ onde $R_{o}=S_{o} \int_{0}^{\infty} A(\tau) d \tau$. A taxa de crescimento $\lambda$ na fase exponencial da epidemia é encontrada como sendo a única raiz real da equação característica, obtida substituindo o "Ansatz" $S^{\prime}(t)=c e^{\lambda t}$ na equação (1.13):

$$
1=S_{o} \int_{0}^{\infty} A(\tau) e^{-\lambda \tau} d \tau
$$


Portanto, temos uma relação implicíta entre $R_{o}$ e $\lambda$. Isso nos motiva a obter uma aproximação para $R_{o}$ a partir do método de geração consecutivas de indíviduos e que nos garantirá uma expressão explicíta para este limiar.

\subsubsection{Formalização do conceito de $R_{o}$ para uma população estruturada}

Quando falamos em populações estruturadas estamos nos referindo a populações heterogêneas. Considere a variável $\xi \in \Omega \subset \mathbb{R}^{m}$, onde $\Omega$ representa o espaço das heterogeneidades. Seja $S=S\left(\xi_{1}\right)$ a função densidade de suscetíveis que descreve a densidade de suscetíveis na ausência de doença. Vamos definir $A\left(\tau, \xi_{1}, \xi_{2}\right)$ como sendo o nível de infectividade esperado de um indivíduo suscetível do tipo $\xi_{1}$ que foi infectado $\tau$ unidades de tempo atrás por um indivíduo do tipo $\xi_{2}$. Assim o número esperado de infecções durante o período infectivo, causado por um infectado por um indivíduo do tipo $\xi_{2}$ é dado por:

$$
\int_{\Omega} S\left(\xi_{1}\right) \int_{0}^{\infty} A\left(\tau, \xi_{1}, \xi 2\right) d \tau d \xi_{1}
$$

Esta quantidade pode ser determinada como o fator de próxima geração de indivíduos infectados. A primeira geração de indivíduos consiste da densidade inicial de indivíduos infectados na população, enquanto que a segunda geração consiste de todas as infecções causadas por membros da primeira geração e assim por diante. Uma vez que novos casos com diferentes características irão surgir, podemos definir uma distribuição de indivíduos sobre o espaço das heterogeneidades $\Omega$ através da função densidade, $\phi$.

Então o operador de próxima geração é definido como

$$
(K(S) \phi)(\xi)=S\left(\xi_{1}\right) \int_{\Omega} \int_{0}^{\infty} A\left(\tau, \xi_{1}, \xi_{2}\right) \phi\left(\xi_{2}\right) d \tau d \xi_{2} .
$$

Este operador nos fornece duas informações: o número de casos secundários resultantes da geração $\phi$ e como esses casos estão distribuídos no espaço $\Omega$. No caso em que $\Omega=\{1, \ldots, m\}$, o operador $K(S)$ é uma matriz $(m \times m)$. No caso em que $m=1$ o valor de $R_{o}$ coincide com o proposto por Kermack e McKendrick, equação (1.2).

Sendo $S$ e $A$ não negativos, podemos interpretar $K$ como um operador positivo no espaço de Banach $L_{1}(\Omega)$ das funções integráveis. Após $m$ gerações a magnitude da população de infectados na aproximação linear é $K(S)^{m} \phi$ e por conseguinte o fator de crescimento por geração é $\left\|K(S)^{m}\right\|^{\frac{1}{m}}$. Para sabermos o que acontece ao longo das gerações consecutivas basta tomarmos $m \rightarrow \infty$.

O raio espectral de $K$ é definido como

$$
\rho(K(S))=\inf _{m \geq 1}\left\|K(S)^{m}\right\|^{\frac{1}{m}}=\lim _{m \rightarrow \infty}\left\|K(S)^{m}\right\|^{\frac{1}{m}}
$$

começando pela zero-ésima geração $\phi$, a m-ésima geração $K(S)^{m} \phi$ converge para zero quando $m \rightarrow \infty$ se $\rho(K(S))<1$.

Além disso, pela positividade e compacidade de $K, \rho(K(S))$ é um autovalor denominado autovalor dominante desde que $|\lambda| \leq r(K(S))$, para todo $\lambda$ no espectro de $K(S)$. O espectro de $K(S)$ denotaremos por $Q_{d}$. Sobre certas condições de irredutibilidade ([DHM90] e [DHR09]) a geração inicial $\phi$ converge para a distribuição estável: 


$$
K(S)^{m} \phi \sim c(\phi) \rho(K)^{m} \psi_{d} \text { para } m \rightarrow \infty,
$$

onde $\psi_{d}$ é o autovetor correspondente a $\rho(K)$ e $c(\phi)$ é um escalar positivo.

Portanto, podemos definir $R_{o}$ como sendo

$$
R_{o}=\rho(K(S))=\text { autovalor dominante de } K(S) \text {. }
$$

Após certo tempo temos que $\psi_{d}$ descreve a distribuição de indivíduos infectados e $\rho(K)$ é o número de casos secundários.

\subsection{Pontos de equilíbrio}

Ao fazermos o estudo do comportamento qualitativo de modelos de propagação de doenças, vamos investigar o comportamento das soluções destes modelos através do estudo do retrato de fase e dos pontos de equilíbrio. No decorrer do nosso estudo, argumentos matemáticos que, independem de sentido epidemiológico serão usados para definir propriedades gerais.

Para o entendimento global do comportamento das trajetórias de fase de um sistema mecânico, analisamos inicialmente os pontos de equilíbrio e as órbitas periódicas. Um dos pioneiros no estudo do retrato de fase de equações diferenciais foi Henry Poincaré, que encontrou sua motivação inicial em problemas da Mecânica Celeste, sendo a estabilidade do sistema solar um dos problemas que despertou sua maior atenção. Esse estudo desenvolvido por Poincaré foi chamado de Teoria Qualitativa ([Tel79]).

Em geral, modelos de propagação mais simples tem dois equilíbrios, sendo eles: o equilíbrio livre de doença e o equilíbrio endemico. As condições de estabilidade destes equilíbrios estão intimamente associadas ao limiar $R_{o}$ uma vez que, o equilíbrio livre de doença será assintoticamente estável se, $R_{o} \leq 1$ e instável se $R_{o}>1$. No caso do equilíbrio endêmico, se $R_{o}>1$ este equilíbrio será assintoticamente estável. Estes resultados serão verificados no capítulo seguinte. 


\section{Capítulo 2}

\section{Estudo Qualitativo de Modelos de Propagação do Dengue}

Este capítulo estabelece uma base teórica para o estudo qualitativo de modelos epidemiológicos. Aqui, objetivamos apresentar resultados importantes da teoria qualitativa de Equações Diferenciais Ordinárias, aplicados ao estudo da estabilidade local e global dos pontos de equilíbrio asociados aos modelos matemáticos de propagação de dengue. A análise que será apresentada baseia-se nos artigos de Esteva e Vargas, em [EV98] e [EV99] e no artigo de Liu e Zhen, em [LJ14].

Embora algumas definições e resultados sejam abordados no apêndice, espera-se que o leitor tenha algum conhecimento prévio sobre a teoria qualitativa de Equações Diferenciais Ordinárias, Sistemas Dinâmicos Monótonos e resultados a eles associados. Para estes tópicos, sugerimos os trabalhos de Sotomayor, em [Tel79], Hirsch, em [HSD12] e Smith, em [Smi95].

O modelo matemático base de propagação da dengue que será estudado, é o modelo compartimental SIR com acoplamento da dinâmica do vetor. Essencialmente, neste capítulo serão desenvolvidas 3 análises. Embora consideremos que o modelo matemático base é o mesmo, iremos trabalhar com diferentes hipóteses sobre a população humana total, $N_{h}(t)$. A primeira análise correspondente ao modelo 1 , considerararemos a hipótese de $N_{h}(t)$ ser constante ao longo do tempo. Na segunda análise, correspondente ao modelo 2, consideraremos a hipótese de $N_{h}(t)$ ser governado por uma função de crescimento exponencial e finalmente, para a última análise, a qual corresponde ao modelo 3, vamos considerar a hipótese de $N_{h}(t)$ ser dado por uma função de crescimento logístico. Quanto a população total de vetores, $N_{v}$, nos modelos 1 e 3 será governada por uma função de crescimento exponencial enquanto que, no modelo 2 , a população total de vetores será mantida constante. Em particular, escolhemos essas funções de crescimento para a população humana, pois estas são aplicadas amplamente na literatura para os mais diversos modelos de propagação de doenças infecciosas. Embora considerando os dados reais a dengue não apresente equilíbrio endêmico devido a sazonalidade, nos interessa verificar teoricamente o impacto dessas hipóteses no cálculo do parâmetro $R_{o}$ e no equilíbrio endêmico.

Neste capítulo, os resultados para cada modelo serão apresentados na seguinte ordem: (i) Faremos um estudo das equações do modelo matemático, verificando condições de existência e unicidade das soluções; (ii) Vamos estimar o parâmetro $R_{o}$; (iii) Obteremos as expressões para os equilíbrios dos modelos em função do parâmetro $R_{o}$ e $(i v)$ Verificaremos condições de estabilidade para os equilíbrios obtidos. 


\section{$2.1 \quad$ Modelo 1}

\subsubsection{Descrição do modelo matemático}

O estudo do modelo matemático apresentado nesta subseção segue a metodologia proposta por Esteva e Vargas, em [EV98]. Este modelo baseia-se na hipótese de que a população humana total é constante ao longo do tempo. O diagrama de fluxo para o modelo 1 é representado pela Figura 2.1. As barras acima das variáveis de estado indicam que estamos trabalhando com as populações em cada compartimento.

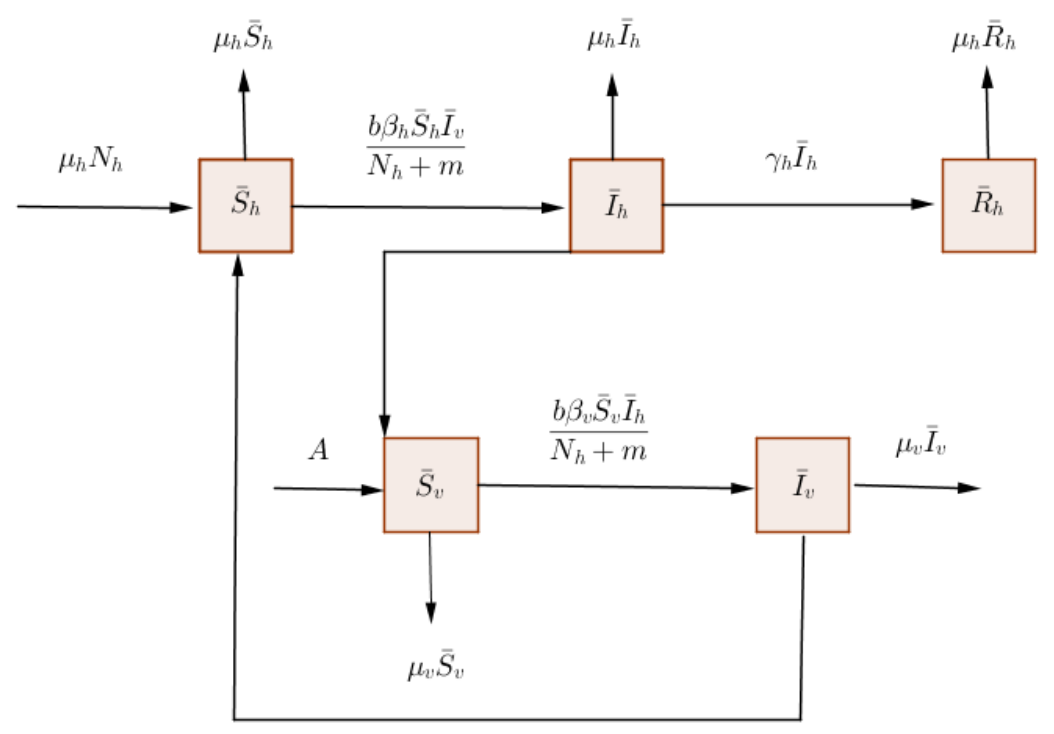

Figura 2.1: Dinâmica de propagação do dengue referente ao modelo 1

Sejam, $N_{h}$ e $N_{v}$ as populações totais de humanos e vetores, respectivamente. Conforme a representação acima, $\bar{S}_{h}, \bar{I}_{h}, \bar{R}_{h}, \bar{S}_{v}$ e $\bar{I}_{v}$ correspondem, respectivamente, as populações de humanos suscetíveis, humanos infectados, humanos recuperados, vetores suscetíveis e vetores infectados. Enquanto a população total humana é mantida constante ao longo do tempo a dinâmica da população de vetores, por outro lado, é governada pela equação:

$$
\frac{d N_{v}}{d t}=A-\mu_{v} N_{v}
$$

em que, $\mu_{v}$ corresponde a taxa de morte dos vetores. A população de vetores, admite uma taxa de recrutamento constante, $A$, que é independente do número atual de vetores adultos. Essa hipótese é considerada razoável, uma vez que, somente um fração do reservatório de larvas e ovos maturam para o estágio adulto e este processo não tem dependência direta da população adulta de mosquitos. Ainda com respeito a dinâmica do vetor, observe que, as soluções de $N_{v}$ se aproximam do equilíbrio $\frac{A}{\mu_{v}}$ quando $t \longrightarrow \infty$.

Na população humana, o parâmetro $\mu_{h}$ corresponde a taxa de nascimentos e mortes de humanos e o parâmetro $\gamma_{h}$ corresponde a taxa de humanos que recuperam-se da doença. A transferência do compartimento suscetível para o compartimento infectado, depende de fatores como: número médio de picadas por vetor, por dia, a probabilidade de transmissão, bem como, o número de suscetíveis 
e infectados de cada espécie.

Neste modelo, o número de picadas do vetor, representado pelo parâmetro $b$, será mantido constante. O parâmetro $m$, corresponde ao número de hospedeiros alternativos como fontes de sangue. Assim, a probabilidade de que um vetor escolha um hospedeiro humano é $\frac{N_{h}}{N_{h}+m}$. Além disso, um humano recebe $b \frac{N_{v}}{N_{h}} \frac{N_{h}}{\left(N_{h}+m\right)}$ picadas por unidade de tempo e um vetor faz $b \frac{N_{h}}{N_{h}+m}$ refeições de sangue humano, logo:

$$
\begin{gathered}
\beta_{h} b \frac{N_{v}}{N_{h}} \frac{N_{h}}{\left(N_{h}+m\right)} \frac{\bar{I}_{v}}{N_{v}}=\frac{\beta_{h} b}{N_{h}+m} \bar{I}_{v}, \\
\beta_{v} b \frac{N_{h}}{N_{h}+m} \frac{\bar{I}_{h}}{N_{h}}=\frac{\beta_{v} b}{N_{h}+m} \bar{I}_{h},
\end{gathered}
$$

representam, as probabilidades de transmissão de vetor para humano e probabilidade de transmissão de humano para vetor respectivamente.

Descritos os parâmetros e variáveis, o modelo 1 é construído sob as seguintes hipóteses:

- As populações de humanos e vetores são distribuídas homogeneamente;

- Todo vetor pode picar um humano com igual probabilidade;

- Humanos que recuperam-se adquirem imunidade permanente contra a doença;

- Somente um sorotipo do vírus da dengue é considerado;

- A taxa de morte causada pela doença é negligenciada;

- Os mosquitos nunca se recuperam de uma infeccção levando em consideração que o seu ciclo de vida do mosquito é muito curto se comparado à expectativa média de vida de um humano;

- Este modelo não considera a transmissão vertical, isto é, todos os humanos recém-nascidos são suscetíveis;

Assim, as equações diferenciais que regem a dinâmica de propagação do dengue para o modelo 1, é descrito pelo sistema abaixo:

$$
\left\{\begin{array}{l}
\frac{d \bar{S}_{h}}{d t}=\mu_{h}\left(N_{h}-\bar{S}_{h}\right)-\frac{\beta_{h} b}{N_{h}+m} \bar{S}_{h} \bar{I}_{v} \\
\frac{d \bar{I}_{h}}{d t}=\frac{\beta_{h} b}{N_{h}+m} \bar{S}_{h} \bar{I}_{v}-\left(\mu_{h}+\gamma_{h}\right) \bar{I}_{h} \\
\frac{d \bar{R}_{h}}{d t}=\gamma_{h} \bar{I}_{h}-\mu_{h} \bar{R}_{h} \\
\frac{d \bar{S}_{v}}{d t}=A-\frac{\beta_{v} b}{N_{h}+m} \bar{S}_{v} \bar{I}_{h}-\mu_{v} \bar{S}_{v} \\
\frac{d \bar{I}_{v}}{d t}=\frac{\beta_{v} b}{N_{h}+m} \bar{S}_{v} \bar{I}_{h}-\mu_{v} \bar{I}_{v}
\end{array}\right.
$$

cujas condições iniciais são: $\bar{S}_{h}(0)=S_{o} \geq 0, \bar{I}_{h}(0)=I_{o} \geq 0, \bar{R}_{h}(0)=R_{o} \geq 0, \bar{S}_{v}(0)=S_{v o} \geq 0$ e $\bar{I}_{v}(0)=I_{v o} \geq 0$.

Com relação ao tamanho das populações de humanos e vetores, temos:

$$
N_{h}=\bar{S}_{h}+\bar{I}_{h}+\bar{R}_{h} \quad N_{v}=\bar{S}_{v}+\bar{I}_{v}
$$


Considere o conjunto $\Gamma$ definido pelas equações (2.2). Observe que, $\Gamma$ é invariante para o sistema (2.1), pois qualquer solução começando em $\Gamma$ satisfaz

$$
\left(\bar{S}_{h}+\bar{I}_{h}+\bar{R}_{h}\right)^{\prime}(t)=0 \quad\left(\bar{S}_{v}+\bar{I}_{v}\right)^{\prime}(t)=\left(\frac{A}{\mu_{v}}\right)^{\prime}=0 .
$$

De fato, como $N_{h}$ é constante e $N_{v}$ tende para $\frac{A}{\mu_{v}}$ pontualmente quando $t$ tende ao infinito, todas as soluções do sistema (2.1) tendem à $\Gamma$. Assim, o estudo do comportamento assintótico das soluções de (2.1) se resume no estudo do comportamento assintótico das soluções em $\Gamma$.

O estudo do comportamento assintótico das soluções de um sistema de equações diferenciais, é motivado essencialmente pelo fato de que a solução analítica dificilmente é obtida para grande parte desses modelos. Podemos extrair informações importantes sobre o comportamento dessas soluções através de uma análise qualitativa, sem que seja preciso conhecer suas soluções analíticamente. O conhecimento das informações sobre o comportamento qualitativo irá nos fornecer indícios sobre a estabilidade dos pontos de equilíbrio destes modelos.

Dado que, em $\Gamma$, tanto as subpopulações de humanos quanto as de vetores permanecem constantes, podemos, sem perda de generalidade, trabalhar com as proporções das populações

$$
S_{h}=\frac{\bar{S}_{h}}{N_{h}}, \quad I_{h}=\frac{\bar{I}_{h}}{N_{h}}, \quad R_{h}=\frac{\bar{R}_{h}}{N_{h}}, \quad S_{v}=\frac{\bar{S}_{v}}{A / \mu_{v}} \quad \text { e } \quad I_{v}=\frac{\bar{I}_{v}}{A / \mu_{v}},
$$

e considerando que $R_{h}=1-S_{h}-I_{h}$ e $S_{v}=1-I_{v}$, podemos obter um sistema tridimensional equivalente ao sistema (2.1):

$$
(F)\left\{\begin{array}{l}
S_{h}^{\prime}(t)=\mu_{h}\left(1-S_{h}\right)-\beta_{h} b \frac{A / \mu_{v}}{N_{h}+m} S_{h} I_{v} \\
I_{h}^{\prime}(t)=\beta_{h} b \frac{A / \mu_{v}}{N_{h}+m} S_{h} I_{v}-\left(\gamma_{h}+\mu_{h}\right) I_{h} \\
I_{v}^{\prime}(t)=\beta_{v} b \frac{N_{h}}{N_{h}+m}\left(1-I_{v}\right) I_{h}-\mu_{v} I_{v},
\end{array}\right.
$$

cuja região de interesse biológico

$$
\Omega=\left\{\left(S_{h}, I_{h}, I_{v}\right) \in \mathbb{R}^{3} / 0 \leq S_{h}, 0 \leq I_{h}, S_{h}+I_{h} \leq 1,0 \leq I_{v} \leq 1\right\}
$$

é positivamente invariante.

Note que, matematicamente, não precisaríamos restringir o domínio de definição das soluções do modelo matemático contudo, pela biologia do problema, soluções definidas fora de $\Omega$ não fariam sentido. A partir de agora, $S_{h}, I_{h}, I_{v}$ referem-se as proporções da população.

Teorema 2.1.1. Assumindo que as condiçôes iniciais encontram-se em $\Omega$, o sistema de equaçôes que descreve o modelo (2.3) tem uma única solução que existe e permanece em $\Omega$ para todo $t>0$.

Prova: O lado direito do sistema (2.3) é contínuo com derivadas parciais contínuas em $\mathbb{R}^{3}$, então (2.3) tem uma única solução. Resta-nos então mostrar que $\Omega$ é positivamente invariante.

i) Suponha $S_{h}=0, I_{h} \geq 0$ e $I_{v} \geq 0$. Isso implica que $S_{h}^{\prime}=\mu_{h}>0$ pois, todos os parâmetros do modelo são não negativos. Logo, o campo de vetores é transversal nesta região; 
ii) Suponha $S_{h}=0, I_{h}>0$ e $I_{v}>0$. Isso implica que $S_{h}^{\prime}=\mu_{h}>0$ pois, todos os parâmetros do modelo são não negativos. Logo, o campo de vetores é transversal nesta região;

iii) Suponha $S_{h} \geq 0, I_{h}=0$ e $I_{v} \geq 0$. Isso implica que $I_{h}^{\prime}=b \beta_{h} \frac{A / \mu_{v}}{N_{h}+m} S_{h} I_{v} \geq 0$ e portanto, o campo de vetores é transversal nesta região;

iv) Suponha $S_{h}>0, I_{h}=0$ e $I_{v}>0$. Isso implica que $I_{h}^{\prime}=b \beta_{h} \frac{A / \mu_{v}}{N_{h}+m} S_{h} I_{v}>0$ e portanto, o campo de vetores é transversal nesta região;

v) Suponha que $S_{h} \geq 0, I_{h} \geq 0$ e $I_{v}=0$ isso implica que $I_{v}^{\prime}=b \beta_{v} \frac{N_{h}}{N_{h}+m} I_{h} \geq 0$ e portanto o campo de vetores é transversal nesta região;

vi) Suponha que $S_{h}>0, I_{h}>0$ e $I_{v}=0$ isso implica que $I_{v}^{\prime}=b \beta_{v} \frac{N_{h}}{N_{h}+m} I_{h}>0$ e portanto o campo de vetores é transversal nesta região;

vii) Suponha que $I_{v}=1$, isso implica $I_{v}^{\prime}=-\mu_{v} \leq 0$ e portanto o campo de vetores neste caso, também é transversal;

viii) Considere a região de $\Omega$ em que $S_{h}+I_{h}=1,0 \leq I_{v} \leq 1$ e $I_{h} \neq 0$. Seja U uma função definida por:

$$
U=S_{h}+I_{h}
$$

Como $S_{h}+I_{h}=1$, observe que os conjuntos de níveis de U são $S_{h}+I_{h}=c$, tais que $c$ é uma constante, são planos paralelos ao plano $S_{h}+I_{h}=1$. Então, ao derivarmos U obteremos:

$$
\begin{aligned}
U^{\prime} & =S_{h}^{\prime}+I_{h}^{\prime} \\
& =\mu_{h}\left(1-S_{h}\right)-\left(\gamma_{h}+\mu_{h}\right) I_{h} \\
& =-\gamma_{h} I_{h}<0 .
\end{aligned}
$$

Portanto, nenhuma das órbitas deixa $\Omega$ e a solução existente é única para todo $t>0$.

\subsubsection{Pontos de equilíbrio e Número de Reprodutibilidade Basal}

Para garantirmos a existência, e exibirmos as expressões para os pontos de equilíbrio do modelo 1, vamos estimar o parâmetro limiar $R_{o}$. Baseados na construção do Operador de Próxima Geração, cuja metodologia é amplamente discutida em Diekmann et al., em [DHM90] e [DHR09], é possível obter $R_{o}$ como sendo

$$
R_{o}=\sqrt{\frac{b^{2} \beta_{v} \beta_{h} A / \mu_{v} N_{h}}{\left(N_{h}+m\right)^{2} \mu_{v}\left(\mu_{h}+\gamma_{h}\right)}} .
$$

Uma definição clássica para o $R_{o}$, associada ao modelo de Ross-McDonald para a malária (descrito por Aron e May, em [AM82], e Anderson e May, em [AM91]), é equivalente a definição proposta por Dieckmann et al, uma vez que o parâmetro de bifurcação, $R_{o}=1$, é o mesmo para ambas as definições. Por questão de simplicidade, usaremos

$$
R_{o}=\frac{b^{2} \beta_{v} \beta_{h} A / \mu_{v} N_{h}}{\left(N_{h}+m\right)^{2} \mu_{v}\left(\mu_{h}+\gamma_{h}\right)}
$$


Dada a expressão para $R_{o}$, obteremos os pontos de equilíbrio do modelo (2.3). Em geral, modelos epidemiológicos que não agregam muitas complexidades, apresentam dois pontos de equilíbrio. $\mathrm{O}$ equilíbrio livre de doença, o qual vamos denotar $E_{1}$, e o equilíbrio endêmico, o qual denotaremos $E_{2}$. O equilíbrio livre de doença, corresponde ao estado de equilíbrio da população na ausência da doença. O equilíbrio endêmico, por outro lado, significa que a doença irá se estabelecer na população por si mesma e permanecerá nela.

Ao analisarmos as equações do sistema (2.3) podemos obter o equilíbrio livre de doença, $E_{1}$ :

$$
E_{1}=(1,0,0)
$$

A obtenção do equilíbrio endêmico, por outro lado, exige algumas manipulações algébricas. Da primeira e terceira equações do sistema (2.3) podemos obter as seguintes relações:

$$
\begin{gathered}
S_{h}=\frac{\beta I_{h}+1}{\left(\beta+M R_{o}\right) I_{h}+1} \mathrm{e} \\
I_{v}=\frac{\beta I_{h}}{\beta I_{h}+1}
\end{gathered}
$$

tais que,

$$
\beta=\frac{b \beta_{v} N_{h}}{\mu_{v}\left(N_{h}+m\right)}, \quad M=\frac{\gamma_{h}+\mu_{h}}{\mu_{h}} \mathrm{e} \quad R_{o}=\frac{b^{2} \beta_{v} \beta_{h} A / \mu_{v} N_{h}}{\left(N_{h}+m\right)^{2} \mu_{v}\left(\mu_{h}+\gamma_{h}\right)}
$$

Substituindo as equações (2.5), (2.6) e usando a relação $\left(\beta b \beta_{h} A / \mu_{v}\right) /\left(N_{h}+m\right)=R_{o}\left(\gamma_{h}+\mu_{h}\right)$ na segunda equação do sistema (2.3), obtém-se que $I_{h}$ deve ser a solução da equação quadrática

$$
-\left(\beta+M R_{o}\right) I_{h}^{2}+\left(R_{o}-1\right) I_{h}=0 .
$$

Substituindo as soluções da equação (2.8), $I_{h}=0$ e $I_{h}=\frac{\left(R_{o}-1\right)}{\beta+M R_{o}}$, nas equações (2.5) e (2.6), obtemos o equilíbrio endêmico, $E_{2}$,

$$
E_{2}=\left(S_{h}^{*}, I_{h}^{*}, I_{v}^{*}\right)
$$

onde,

$$
S_{h}^{*}=\frac{\beta+M}{\beta+M R_{o}} \quad, \quad I_{h}^{*}=\frac{R_{o}-1}{\beta+M R_{o}} \quad \text { e } \quad I_{v}^{*}=\frac{\beta\left(R_{o}-1\right)}{R_{o}(\beta+M)}
$$

Se $R_{o} \leq 1$ o equilíbrio livre de doença será o único equilíbrio do sistema (2.3) em $\Omega$. Se $R_{o}>1$ o equilíbrio endêmico também estará presente em $\Omega$.

\subsubsection{Análise de estabilidade dos pontos de equilíbrio}

Considere as soluções do sistema (2.3) dadas pelos dois pontos de equilíbrio $E_{1}$ e $E_{2}$. A grosso modo, a estabilidade assintótica dos equilíbrios significa que soluções começando próximas o suficiente dos equilíbrios permanecem próximas e se aproximam destes equilíbrios quando $t \rightarrow \infty$, 
enquanto que a instabilidade do equilíbrio singifica que soluções começando arbitrariamente próximas destes equilíbrios não necessariamente se aproximam deles ao longo do tempo.

Dado que o modelo em estudo descreve a evolução de um processo biológico, as soluções estáveis adquirem um importância essencial no estudo destes modelos. Particularmente, estamos interessados em verificar a estabilidade assintótica do equilíbrio endêmico e com isso garantir que as frações das populações em cada um dos compartimentos se aproxima de um estado estacionário.

Dividiremos o estudo da estabilidade dos pontos de equilíbrio em três etapas. Inicialmente verificaremos a estabilidade local do equilíbrio livre de doença e do equilíbrio endêmico através do critério de Routh-Hurwitz. Em seguida, faremos o estudo da estabilidade global do equilíbrio livre de doença. Para verificarmos a estabilidade global deste equilíbrio faremos a construção de uma função de Lyapunov e concluiremos usando o Princípio da Invariância de Lasalle. Por fim, passaremos a prova da estabilidade global do equilíbrio endêmico. Nesta prova verificaremos que o modelo 1 satisfaz as seguintes condições: competitividade, persistência, propriedade de PoincaréBendixson para sistemas competitivos e admite a propriedade da estabilidade orbital de órbitas periódicas.

Iniciaremos então pela análise da estabilidade local de $E_{1}=(1,0,0)$. Seja, $D F\left(E_{1}\right)$ a derivada do campo de vetores associado ao sistema (2.3), aplicada à $E_{1}$ :

$$
D F\left(E_{1}\right)=\left[\begin{array}{ccc}
-\mu_{h} & 0 & -\frac{b \beta_{h} A / \mu_{v}}{N_{h}+m} \\
0 & -\left(\gamma_{h}+\mu_{h}\right) & \frac{b \beta_{h} A / \mu_{v}}{N_{h}+m} \\
0 & \frac{b \beta_{v} N_{h}}{N_{h}+m} & -\mu_{v}
\end{array}\right] .
$$

Calculando o polinômio característico de grau 3, associado a matriz (2.10), temos:

$$
p(\lambda)=\left(\mu_{h}+\lambda\right) q(\lambda),
$$

onde, $q(\lambda)=\left[-\left(\gamma_{h}+\mu_{h}+\mu_{v}\right)\left(\mu_{v}+\lambda\right)+\frac{b^{2} \beta_{h} \beta_{v} A / \mu_{v} N_{h}}{\left(N_{h}+m\right)^{2}}\right]$.

Claramente uma das raízes da equação (2.11) é $\lambda_{1}=-\mu_{h}$. Analisando $q(\lambda)$, podemos concluir através do critério de Routh-Hurwitz (consultar Apêndice A, Teorema A.1.11) que todas as raízes da equação (2.11) são negativas ou têm parte real negativa se $R_{o}<1$.

Portanto, se $R_{o}<1$ o equilíbrio livre de doença, $E_{1}$, é localmente assintoticamente estável.

Note que, para $R_{o}>1$, o equilíbrio livre de doença $E_{1}$, que até então se mantinha estável, tornase-á um ponto de equilíbrio instável hiperbólico. Isso ocorre, devido ao aparecimento do equilíbrio endêmico, $E_{2}$, que receberá a estabilidade que até então era de $E_{1}$ em $\Omega$.

Além da estabilidade local, nos interessa verificar condições sobre a estabilidade global de $E_{1}$. A estabilidade global dos pontos de equilíbrio é importante, pois nos garante que, independentemente do tamanho das subpopulações de cada uma das classes, as soluções do modelo matemático irão convergir para o equilíbrio.

Para provarmos a estabilidade global de $E_{1}$, faremos a construção de uma função de Lyapunov e concluiremos a prova usando o Princípio da Invariância de Lasalle ([HSD12]). Com isso, mostraremos que se $R_{o} \leq 1$, então $E_{1}$ é globalmente assintoticamente estável. 
Teorema 2.1.2 (Princípio da Invariância de Lasalle). Seja $x^{*}$ um ponto de equilíbrio para o sistema $x^{\prime}=F(x), U \in \mathbb{R}^{n}$ é um conjunto aberto contendo $x^{*}$ e seja $L: U \rightarrow \mathbb{R}$ uma função de Lyapunov para $x^{*}$. Seja $P \in U$ uma vizinhança fechada de $x^{*}$. Suponha que $P$ é positivamente invariante $e$ que não existe solução inteira em $P-x^{*}$ em que L é constante. Então $x^{*}$ é assintoticamente estável e P está contida na bacia de atração de $x^{*}$.

Prova: A demonstração deste resultado pode ser obtida em [HSD12], p.201.

Seja V, a função de Lyapunov definida por:

$$
V(t)=\frac{b \beta_{h} A / \mu_{v}}{\left(N_{h}+m\right) \mu_{v}} I_{v}(t)+I_{h}(t) \geq 0 .
$$

Calculando a derivada de $\mathrm{V}$ com relação à t, obtemos:

$$
\begin{aligned}
\frac{d V}{d t} & =\frac{b \beta_{h} A / \mu_{v}}{\left(N_{h}+m\right) \mu_{v}} \frac{d I_{v}}{d t}+\frac{d I_{h}}{d t} \\
& =\frac{b \beta_{h} A / \mu_{v}}{\left(N_{h}+m\right) \mu_{v}}\left[\frac{b \beta_{v} N_{h}}{N_{h}+m}\left(1-I_{v}(t)\right) I_{h}(t)-\mu_{v} I_{v}(t)\right]+\left[\frac{b \beta_{h} A / \mu_{v}}{N_{h}+m} S_{h}(t) I_{v}(t)-\left(\gamma_{h}+\mu_{h}\right) I_{h}(t)\right] \\
& =-\frac{b \beta_{h} A / \mu_{v}}{N_{h}+m}\left(1-S_{h}(t)\right) I_{v}(t)-\left(\gamma_{h}+\mu_{h}\right)\left[1-R_{o}\left(1-I_{v}(t)\right)\right] I_{h}(t) \leq 0 \quad \text { em } \quad \Omega
\end{aligned}
$$

pois, $I_{h} \geq 0, S_{h} \geq 0,0 \leq I_{v} \leq 1, I_{h} \leq 1-S_{h}$ e $R_{o} \leq 1$. Logo, $V^{\prime}(t) \leq 0$ em $\Omega$ para $R_{o} \leq 1$.

O subconjunto de $\Omega$, tal que $V^{\prime}=0$, é definido pelas equações:

$$
\begin{array}{lll}
\left(1-S_{h}\right) I_{v}=0 & , \quad I_{h}=0 \quad \text { se } \quad R_{o}<1 \quad \text { e } \\
\left(1-S_{h}\right) I_{v}=0 & , \quad I_{v} I_{h}=0 \quad \text { se } \quad R_{o}=1 .
\end{array}
$$

Analisando o sistema (2.3), podemos concluir que o maior conjunto invariante compacto em que $\left\{\left(S_{h}, I_{h}, I_{v}\right): V^{\prime}=0\right\}$ é $\left\{E_{1}\right\}$. Portanto, pelo Princípio da Invariância de Lasalle concluímos que $E_{1}$ é globalmente assintoticamente estável para $R_{o} \leq 1$.

Estabelecidas as condições de estabilidade sobre o equilíbrio livre de doença, faremos o mesmo para o equilíbrio endêmico. Mostraremos que se $R_{o}>1$ o equilíbrio $E_{2}$ é localmente estável. Para isso, usaremos novamente o critério de Routh-Hurwitz.

Considere a matriz $D F\left(E_{2}\right)$ como sendo a linearização do sistema (2.3) em torno do equilíbrio endêmico $E_{2}$,

$$
D F\left(E_{2}\right)=\left[\begin{array}{ccc}
-\mu_{h}\left(\frac{\beta+M R_{o}}{\beta+M}\right) & 0 & -\frac{\mu_{h} M R_{o}}{\beta}\left(\frac{\beta+M}{\beta+M R_{o}}\right) \\
\frac{\mu_{h} M\left(R_{o}-1\right)}{\beta+M} & -\mu_{h} M & \frac{\mu_{h} M R_{o}}{\beta}\left(\frac{\beta+M}{\beta+M R_{o}}\right) \\
0 & \frac{\mu_{v} \beta}{R_{o}}\left(\frac{\beta+M R_{o}}{\beta+M}\right) & -\mu_{v} R_{o}\left(\frac{\beta+M}{\beta+M R_{o}}\right)
\end{array}\right] .
$$

cujos, $\beta$, M e $R_{o}$ são dados pelas equações (2.7).

Seja $P(\lambda)$, o polinômio característico associado a matriz (2.14), dado por: 


$$
\begin{aligned}
P(\lambda) & =\lambda^{3}+\left[\mu_{h}\left(\frac{\beta+M R_{o}}{\beta+M}\right)+\mu_{h} M+\mu_{v} R_{o}\left(\frac{\beta+M}{\beta+M R_{o}}\right)\right] \lambda^{2} \\
& +\left[\mu_{h}^{2} M\left(\frac{\beta+M R_{o}}{\beta+M}\right)+\mu_{h} \mu_{v} R_{o}+\mu_{h} \mu_{v} M R_{o}\left(\frac{\beta+M}{\beta+M R_{o}}\right)-\mu_{h} \mu_{v} M\right] \lambda \\
& +\left[\mu_{h}^{2} \mu_{v} M R_{o}+\frac{\mu_{h}^{2} \mu_{v} M^{2}}{\beta+M}\left(R_{o}-1\right)-\mu_{h}^{2} \mu_{v} M\left(\frac{\beta+M R_{o}}{\beta+M}\right)\right] .
\end{aligned}
$$

Sendo, $P(\lambda)$ da forma:

$$
P(\lambda)=a_{o} \lambda^{3}+a_{1} \lambda^{2}+a_{2} \lambda+a_{3},
$$

temos:

$$
\begin{aligned}
a_{o} & =1 \\
a_{1} & =\mu_{h}\left(\frac{\beta+M R_{o}}{\beta+M}\right)+\mu_{h} M+\mu_{v} R_{o}\left(\frac{\beta+M}{\beta+M R_{o}}\right) \\
a_{2} & =\mu_{h}^{2} M\left(\frac{\beta+M R_{o}}{\beta+M}\right)+\mu_{h} \mu_{v} R_{o}+\frac{\mu_{h} \mu_{v} M \beta\left(R_{o}-1\right)}{\beta+M R_{o}} \\
a_{3} & =\mu_{h}^{2} \mu_{v} M\left[R_{o}+\left(\frac{M\left(R_{o}-1\right)}{\beta+M}\right)-\left(\frac{\beta+M R_{o}}{\beta+M}\right)\right] \\
& =\mu_{h}^{2} \mu_{v} M\left(R_{o}-1\right) .
\end{aligned}
$$

Verificando as hipóteses do critério de Routh-Hurwitz temos que, $E_{2}$ é localmente assintoticamente estável. De fato, $a_{o}>0, a_{1}, a_{2}, a_{3}>0$ pois, $R_{o}>1$ e além disso, todos os parâmetros do modelo são não negativos. Resta-nos então mostrar que, se $R_{o}>1$, então $a_{1} a_{2}>a_{0} a_{3}$. Assim,

$$
\begin{aligned}
a_{1} a_{2} & =\mu_{h}^{3} M\left(\frac{\beta+M R_{o}}{\beta+M}\right)^{2}+\mu_{h}^{2} \mu_{v} R_{o}\left(\frac{\beta+M R_{o}}{\beta+M}\right)+\mu_{h}^{2} \mu_{v} M \beta\left(\frac{R_{o}-1}{\beta+M}\right) \\
& +\mu_{h}^{3} M^{2}\left(\frac{\beta+M R_{o}}{\beta+M}\right)+\mu_{h}^{2} \mu_{v} M R_{o}+\mu_{h}^{2} \mu_{v} M^{2} \beta\left(\frac{R_{o}-1}{\beta+M R_{o}}\right) \\
& +\mu_{h}^{2} \mu_{v} M R_{o}+\mu_{h} \mu_{v}^{2} R_{o}^{2}\left(\frac{\beta+M}{\beta+M R_{o}}\right)+\mu_{h} \mu_{v}^{2} M \beta R_{o} \frac{\left(R_{o}-1\right)(\beta+M)}{\left(\beta+M R_{o}\right)^{2}} \\
& >\mu_{h}^{2} \mu_{v} M R_{o}>a_{o} a_{3}=\mu_{h}^{2} \mu_{v} M R_{o}-\mu_{h}^{2} \mu_{v} M
\end{aligned}
$$

Portanto, é possível concluir que, para $R_{o}>1$, o equilíbrio endêmico $E_{2}$ é localmente assintoticamente estável.

Usando argumentos da teoria de sistemas dinâmicos monótonos iremos provar que o equilíbrio endêmico é globalmente estável. Em particular, garantir a estabilidade global do equilíbrio endêmico tem sido um desafio para a maior parte dos modelos epidemiológicos. Essa dificuldade ocorre, principalmente pelo fato de que grande parte dos modelos epidemiológicos possuem dimensão alta $(\geq 3)$ e tratam-se de sistemas autônomos não lineares, para os quais a teoria clássica de Poincaré-Bendixson não é válida ([CCS04]). 
Contudo, a extensão da teoria de Poincaré-Bendixson de dimensão 2 para dimensões maiores tornouse uma ferramenta muito utilizada na análise da estabilidade global deste equilíbrio. Para uma classe de sistemas dinâmicos tridimensionais que apresentam monotonicidade, a propriedade de Poincaré-Bendixson é preservada e a existência de soluções periódicas não constantes pode ser descartada através da estabilidade de órbitas periódicas ([LM95], [Mu190], [Smi95]). Essa extensão da teoria de Poincaré-Bendixson para sistemas competitivos tridimensionais foi provada por Hirsch, em [Hir90] e Smith, em [Smi88].

Para enunciarmos o teorema principal precisamos de algumas definições. Seja $D \in \mathbb{R}^{n}$ um conjunto aberto e seja $x \longmapsto f(x) \in \mathbb{R}^{n}$ uma função de classe $C^{1}$ definida em D. Considere o sistema autônomo em $\mathbb{R}^{n}$ dado por:

$$
x^{\prime}=f(x) .
$$

Definição 2.1.3. O sistema (2.17) é dito competitivo em D se, para alguma matriz diagonal $H=\operatorname{diag}\left(\epsilon_{1}, \epsilon_{2}, \cdots, \epsilon_{n}\right)$, onde cada $\epsilon_{i}, i=1, n$ é 1 ou -1 e $H(D F(x)) H$ tem elementos não positivos fora da diagonal para $x \in D$ e, DF $(x)$ é a matriz jacobiana associada ao sistema (2.17).

Uma das características importantes de sistemas tridimensionais competitivos é que possuem a propriedade de Poincaré-Bendixson:

Teorema 2.1.4 (Propriedade de Poincaré-Bendixson para sistemas competitivos e cooperativos tridimensionais). Um conjunto $\omega$-limite compacto de um sistema competitivo ou cooperativo em $\mathbb{R}^{3}$ que não contém pontos de equilíbrio é uma órbita periódica.

Definição 2.1.5. O sistema (2.17) é dito persistente se, e somente se, cada solução $x(t)$ de (2.17) que começa no $\operatorname{int}(D)$ tem a propriedade $\liminf _{t \rightarrow \infty} x(t)$, está a uma distância positiva da fronteira de $D$.

Definição 2.1.6. Suponha que o sistema (2.17) tenha uma solução periódica $\gamma(t)$ com período minimal $\omega>0$ e órbita $\gamma=\{\gamma(t): 0 \leq t \leq \omega\}$. Esta órbita é dita orbitalmente estável se, e somente se, para cada $\varepsilon>0$, existe um $\delta>0$ tal que qualquer solução $x(t)$ de (2.17), para o qual a distância entre $x(0)$ e $\gamma$ é menor que $\delta$, permanece a uma distância inferior a $\varepsilon$ de $\gamma, \forall t \geq 0$. Além disso, uma órbita é dita assintoticamente estável se, a distância de $x(t)$ a $\gamma$ também tende a zero quando $t$ tende ao infinito.

Introduziremos agora um conceito proposto por Muldowney, em [Mul90], que estabelece uma condição suficiente para a estabilidade orbital assintótica de órbitas periódicas.

Definição 2.1.7. O sistema (2.17) tem a propriedade da estabilidade de órbitas periódicas se, e somente se, a órbita de qualquer solução periódica $\gamma(t)$ se existir é orbitalmente assintoticamente estável.

Definição 2.1.8. Seja $A=\left(a_{i j}\right)$ uma matriz de dimensão $(n \times n)$ e $1 \leq k \leq n$ um inteiro, a $k-e ́ s i m a$ matriz composta aditiva de A é denominada $A^{[k]}$. Esta é uma matriz $N \times N, N=\left(\begin{array}{l}n \\ k\end{array}\right)$ é definida por:

$$
A^{[k]}=\left.D_{+}(I+h A)^{(k)}\right|_{h=0}
$$

onde, $D_{+}$denota a derivada pelo lado direito com relação a h e I corresponde a matriz identidade.

Teorema 2.1.9. Uma condição suficiente para que uma órbita periódica $\gamma=\{\gamma(t): 0 \leq t \leq D\}$ de $x^{\prime}=F(x)$ ser assintoticamente estável é que o sistema linear

$$
z^{\prime}=\frac{D F}{d x}^{[2]}(\gamma(t)) z
$$

seja assintoticamente estável.

Prova: Ver Muldowney [Mul90].

Agora temos condições sufucientes para enunciar e provar o seguinte resultado: 
Teorema 2.1.10. Assuma $n=3$ e D convexo e limitado. Suponha que o sistema (2.17) satisfaz a propriedade de Poincaré-Bendixson, é persistente e tem a propriedade da estabilidade de órbitas periódicas. Se $E_{2}$ é o único equilíbrio no int $(D)$ e se $E_{2}$ é localmente assintoticamente estável então, E $E_{2}$ é globalmente assintoticamente estável.

Prova: A prova deste teorema pode ser obtida em [LM95].

Observe que, de acordo com o teorema 2.1.10, $E_{2}$ será globalmente estável para $R_{o}>1$ se mostrarmos que o sistema (2.3) é competivo e portanto preserva a propriedade de Poincaré-Bendixson, é persistente para $R_{o}>1$ e satisfaz a propriedade da estabilidade de órbitas periódicas.

Com o intuito de verificar essas hipóteses, provaremos as afirmações subsequentes.

Afirmação 2.1.11. O sistema (2.3) é competitivo.

Prova: Pela Definição 2.1.3, o sistema (2.3) é dito competitivo em $\Omega$ se, para alguma matriz diagonal $H=\operatorname{diag}\left(\epsilon_{1}, \epsilon_{2}, \cdots, \epsilon_{n}\right)$, cujos, $\epsilon_{i}, i=1, n$ é 1 ou $-1, H(D F(x)) H$ tem elementos não positivos fora da diagonal para $x \in \Omega$ e $D F(x)$ é a matriz jacobiana associada ao sistema (2.3).

Assim, calculando a matriz jacobiana do sistema (2.3),

$$
D F=\left[\begin{array}{ccc}
-\mu_{h}-\frac{b \beta_{h} A / \mu_{v} I_{v}}{N_{h}+m} & 0 & -\frac{b \beta_{h} A / \mu_{v} S_{v}}{N_{h}+m} \\
\frac{b \beta_{h} A / \mu_{v} I_{v}}{N_{h}+m} & -\left(\gamma_{h}+\mu_{h}\right) & \frac{b \beta_{h} A / \mu_{v} S_{h}}{N_{h}+m} \\
0 & \frac{b \beta_{v} N_{h}\left(1-I_{v}\right)}{N_{h}+m} & -\frac{b \beta_{v} N_{h} I_{h}}{N_{h}+m}-\mu_{v}
\end{array}\right]
$$

e, escolhendo H como sendo:

$$
H=\left[\begin{array}{ccc}
1 & 0 & 0 \\
0 & -1 & 0 \\
0 & 0 & 1
\end{array}\right]
$$

vamos obter:

$$
\begin{aligned}
H(D F) H= & {\left[\begin{array}{ccc}
1 & 0 & 0 \\
0 & -1 & 0 \\
0 & 0 & 1
\end{array}\right] \cdot\left[\begin{array}{ccc}
-\mu_{h}-\frac{b \beta_{h} A / \mu_{v} I_{v}}{N_{h}+m} & 0 & -\frac{b \beta_{h} A / \mu_{v} S_{v}}{N_{h}+m} \\
\frac{b \beta_{h} A / \mu_{v} I_{v}}{N_{h}+m} & -\left(\gamma_{h}+\mu_{h}\right) & \frac{b \beta_{h} A / \mu_{v} S_{h}}{N_{h}+m} \\
0 & \frac{b \beta_{v} N_{h}\left(1-I_{v}\right)}{N_{h}+m} & -\frac{b \beta_{v} N_{h} I_{h}}{N_{h}+m}-\mu_{v}
\end{array}\right] \cdot\left[\begin{array}{ccc}
1 & 0 & 0 \\
0 & -1 & 0 \\
0 & 1
\end{array}\right] } \\
= & {\left[\begin{array}{ccc}
-\mu_{h}-\frac{b \beta_{h} A / \mu_{v} I_{v}}{N_{h}+m} & -\frac{b \beta_{h} A / \mu_{v} S_{v}}{N_{h}+m} \\
-\frac{b \beta_{h} A / \mu_{v} I_{v}}{N_{h}+m} & -\left(\gamma_{h}+\mu_{h}\right) & -\frac{b \beta_{h} A / \mu_{v} S_{h}}{N_{h}+m} \\
0 & -\frac{b \beta_{v} N_{h}\left(1-I_{v}\right)}{N_{h}+m} & -\frac{b \beta_{v} N_{h} I_{h}}{N_{h}+m}-\mu_{v}
\end{array}\right] }
\end{aligned}
$$

Com isso podemos concluir que o sistema (2.3) é competitivo em $\Omega$ com relação a ordenação parcial definida pelo octante (ver Apêndice A.2)

$$
K=\left\{\left(S_{h}, I_{h}, I_{v}\right) \in \mathbb{R}^{3}: S_{h} \geq 0, I_{h} \leq 0, I_{v} \geq 0\right\}
$$


Afirmação 2.1.12. O sistema (2.3) é persistente.

De acordo com Butler et al., em [BFW86], o sistema (2.3) é persistente se verificarmos a seguinte proposição:

Proposição 2.1.13. Na fronteira de $\Omega$, o sistema (2.3) tem somente um ponto $\omega$-limite que é o equilíbrio livre de doença, $E_{1}$. Além disso, para $R_{o}>1, E_{1}$ não pode ser $\omega$-limite de qualquer órbita no int $(\Omega)$.

Prova: Inicialmente, observe que o campo de vetores associado ao sistema (2.3) é transversal na fronteira de $\Omega$ exceto no eixo $S_{h}$ pois, como foi mostrado no teorema 2.1.1 o eixo $S_{h}$ é invariante pelo fluxo do sistema (2.3). Assim, restritos ao eixo $S_{h}$ temos:

$$
S_{h}^{\prime}(t)=\mu_{h}\left(1-S_{h}(t)\right)
$$

cuja solução é

$$
S_{h}(t)=c e^{-\mu_{h} t}+1
$$

$\operatorname{logo} S_{h} \rightarrow 1$ quando $t \rightarrow \infty$ e portanto, $E_{1}=(1,0,0)$ é o único $\omega$-limite na fronteira de $\Omega$.

Para mostrarmos a segunda parte da proposição, considere a função:

$$
V(t)=I_{v}(t)+\frac{\mu_{v}\left(N_{h}+m\right)\left(1+R_{o}\right)}{2 b \beta_{h} A / \mu_{v}} I_{h}(t) .
$$

Derivando (2.19) com relação à $t$, vamos obter:

$$
\begin{aligned}
V^{\prime}(t) & =I_{v}^{\prime}(t)+\frac{\mu_{v}\left(N_{h}(t)+m\right)\left(1+R_{o}\right)}{2 b \beta_{h} A / \mu_{v}} I_{h}^{\prime}(t) \\
& =\frac{b \beta_{v} N_{h}(t)}{N_{h}(t)+m}\left(1-I_{v}(t)\right) I_{h}(t)-\mu_{v} I_{v}(t) \\
& +\frac{\mu_{v}\left(N_{h}(t)+m\right)\left(1+R_{o}\right)}{2 b \beta_{h} A / \mu_{v}}\left[\frac{b \beta_{h} A / \mu_{v}}{N_{h}(t)+m} S_{h}(t) I_{v}(t)-\left(\gamma_{h}+\mu_{h}\right) I_{h}(t)\right] \\
& =\left[\left(1-I_{v}(t)\right)-\frac{1}{2}\left(\frac{1}{R_{o}}+1\right)\right] b \beta_{v} \frac{N_{h}(t)}{N_{h}(t)+m} I_{h}(t)+\left[S_{h}(t)-\frac{2}{1+R_{o}}\right] \frac{\mu_{v}\left(1+R_{o}\right)}{2} I_{v}(t) .
\end{aligned}
$$

Como $R_{o}>1$, temos $\frac{1}{2}\left(\frac{1}{R_{o}}\right)<1$ e $\frac{2}{1+R_{o}}<1$ e portanto, existe uma vizinhança $\mathrm{U}$ de $E_{1}$ tal que, para todo $\left(S_{h}, I_{h}, I_{v}\right) \in U \cap \operatorname{int}(\Omega)$ as expressões entre colchetes são positivas e por conseguinte $V^{\prime}(t)>0$ nessa vizinhança $\mathrm{U}$, exceto no ponto $(1,0,0)$.

Observe também que os conjuntos de nível de $\mathrm{V}$ são os planos

$$
I_{v}(t)+\frac{\mu_{v}\left(N_{h}(t)+m\right)\left(1+R_{o}\right)}{2 b \beta_{h} A / \mu_{v}} I_{h}(t)=c,
$$

que se afastam do eixo $S_{h}$ conforme $c$ cresce. Como $\mathrm{V}$ cresce ao longo de suas órbitas, partindo de $U \cap \operatorname{int}(\Omega)$, concluímos que as órbitas se afastam de $E_{1}$ quando t cresce. Portanto, para $R_{o}>1, E_{1}$ não pode ser $\omega$-limite de qualquer órbita no $\operatorname{int}(\Omega)$

Assim, concluímos que o sistema (2.3) é persistente.

Uma vez verificado que o sistema (2.3) é competitivo ele admite a propriedade de Poincaré-Bendixson:

Afirmação 2.1.14. Qualquer conjunto $\omega$-limite do sistema (2.3), no interior de $\Omega$ é uma órbita fechada ou o equilíbrio endêmico $E_{2}$. 
Prova: Suponha que $\gamma$ é um conjunto $\omega$-limite no interior de $\Omega$. Se $\gamma$ não contém $E_{2}$, então $\gamma$ não contém nenhum equilíbrio uma vez que, $E_{2}$ é o único equilíbrio no interior de $\Omega$. Portanto, pelo Teorema 2.1.4 um conjunto que não contém equilíbrios é uma órbita fechada. Isso implica que $\gamma$ é uma órbita fechada. Por outro lado, se $\gamma$ contém $E_{2}$, então, para qualquer órbita de $\gamma$ que esteja arbitrariamente próxima de $E_{2}$, convergirá para $E_{2}$ uma vez que, $E_{2}$ é localmente assintoticamente estável. Então $\gamma=E_{2}$.

Observe que, o fato do sistema (2.3) ser persistente, implica que o comportamento das soluções do sistema está bem definido no bordo de $\Omega$ e além disso o equilíbrio livre de doença, que já mostramos ser globalmente estável, não pode ser $\omega$-limite de qualquer órbita no interior de $\Omega$. Consequentemente, o equilíbrio endêmico é o único equilíbrio no interior de $\Omega$ e ainda, $E_{1}$ não pode ser $\omega$-limite de qualquer órbita no interior de $\Omega$.

Entretanto, pelo fato do sistema (2.3) ser competitivo e por sua vez, admitir a propriedade de PoincaréBendixson, podemos ter órbitas periódicas no interior de $\Omega$ além do equilíbrio endêmico, conforme a Afirmação 2.1.14. Portanto, precisamos mostrar que não existem órbitas periódicas no interior de $\Omega$.

Suponha que exista um solução periódica $\gamma$ no interior de $\Omega$. Como esta solução é uma curva periódica, sabemos que a órbita de uma curva periódica é a própria curva. Pela Definição 2.1.7, se existir uma solução periódica no interior de $\Omega$, sua órbita deve ser assintoticamente estável. Portanto, $\gamma$ é assintoticamente estável.

Como $\gamma$ é estável no interior de $\Omega$, o campo de vetores deve apontar para a curva. Por outro lado, mostramos que $E_{2}$ é localmente estável, isso implica que existe uma vizinhança de $E_{2}$ onde, o campo de vetores aponta para este equilíbrio. Particularmente, para que ambas situações ocorram no interior de $\Omega$, deve existir uma curva fechada $\gamma_{1}$, entre $\gamma$ e $E_{2}$, que seja instável, entretanto, isso é um absurdo pois, $\gamma_{1}$ deve ser estável.

Embora possamos aplicar o mesmo argumento várias vezes, sempre vamos ter um região em que o campo de vetores aponta para lados opostos, exigindo a existência de uma curva periódica, cuja órbita seja instável. Portanto, precisamos mostrar que o sistema (2.3), possui a propriedade da estabilidade de óbitas periódicas.

Conforme o Teorema 2.1.9, uma condição suficiente para verificarmos se o sistema (2.3), possui a propriedade da estabilidade de órbitas periódicas, consiste na prova da seguinte afirmação:

Afirmação 2.1.15. O sistema (2.3) tem a propriedade da estabilidade de órbitas periódicas.

Prova: Seja $\gamma(t)$ uma órbita periódica do sistema (2.3). Seja DF a diferencial do campo de vetores associado ao sistema (2.3). Se mostrarmos que:

$$
z^{\prime}=\frac{D F}{d x}^{[2]}(\gamma(t)) z
$$

é estável, então pelo teorema 2.1.9, $\gamma(t)$ é assintoticamente estável.

Seja DF a matriz jacobiana do sistema (2.3)

$$
D F=\left[\begin{array}{ccc}
-\mu_{h}-\frac{b \beta_{h} A / \mu_{v} I_{v}}{N_{h}+m} & 0 & -\frac{b \beta_{h} A / \mu_{v} S_{v}}{N_{h}+m} \\
\frac{b \beta_{h} A / \mu_{v} I_{v}}{N_{h}+m} & -\left(\gamma_{h}+\mu_{h}\right) & \frac{b \beta_{h} A / \mu_{v} S_{h}}{N_{h}+m} \\
0 & \frac{b \beta_{v} N_{h}\left(1-I_{v}\right)}{N_{h}+m} & -\frac{b \beta_{v} N_{h} I_{h}}{N_{h}+m}-\mu_{v}
\end{array}\right] .
$$

Substituindo as expressões, $a_{h}=\frac{A / \mu_{v}}{N_{h}+m}$ e $a_{v}=\frac{N_{h}}{N_{h}+m}$ na matriz jacobiana, temos:

$$
D F=\left[\begin{array}{ccc}
-\left(\mu_{h}+b \beta_{h} a_{h} I_{v}\right) & 0 & -b \beta_{h} a_{h} S_{h} \\
b \beta_{h} a_{h} I_{v} & -\left(\gamma_{h}+\mu_{h}\right) & b \beta_{h} a_{h} S_{h} \\
0 & b \beta_{v} a_{v}\left(1-I_{v}\right) & -\left(b \beta_{v} a_{v} I_{h}+\mu_{v}\right)
\end{array}\right]
$$


Conforme Li e Muldowney [Mul90], a matriz $D F^{[2]}$, é dada por:

$$
D F^{[2]}=\left[\begin{array}{ccc}
-\left(2 \mu_{h}+a_{h} b \beta_{h} I_{v}+\gamma_{h}\right) & a_{h} b \beta_{h} S_{h} & a_{h} b \beta_{h} S_{h} \\
a_{v} b \beta_{v}\left(1-I_{v}\right) & -\left(\mu_{h}+a_{h} b \beta_{h} I_{v}+\mu_{v}+a_{v} b \beta_{v} I_{h}\right) & 0 \\
0 & a_{h} b \beta_{h} I_{v} & -\left(\gamma_{h}+\mu_{h}+\mu_{v}+a_{v} b \beta_{v} I_{h}\right)
\end{array}\right] .
$$

Portanto, para avaliarmos a estabilidade de $\gamma(t)$, a equação (2.20) torna-se:

$$
\left\{\begin{array}{l}
X^{\prime}=-\left(\mu_{h}+a_{h} b \beta_{h} I_{v}+\gamma_{h}+\mu_{h}\right) X+\left(a_{h} b \beta_{h} S_{h}\right) Y+\left(a_{h} b \beta_{h} S_{h}\right) Z \\
Y^{\prime}=\left(a_{v} b \beta_{v}\left(1-I_{v}\right)\right) X-\left(\mu_{h}+a_{h} b \beta_{h} I_{v}+\mu_{v}+a_{v} b \beta_{v} I_{h}\right) Y \\
Z^{\prime}=\left(a_{h} b \beta_{h} I_{v}\right) Y-\left(\gamma_{h}+\mu_{h}+\mu_{v}+a_{v} b \beta_{v} I_{h}\right) Z
\end{array}\right.
$$

Note que, a partir de agora, avaliar a estabilidade assintótica da equação (2.20) corresponde, equivalentemente, à avaliar a estabilidade assintótica do sistema (2.22). Para isso, usaremos a seguinte função de Lyapunov,

$$
V\left(X(t), Y(t), Z(t), S_{h}(t), I_{h}(t), I_{v}(t)\right)=\left\|\left(X(t), \frac{I_{h}(t)}{I_{v}(t)} Y(t), \frac{I_{h}(t)}{I_{v}(t)} Z(t)\right)\right\|
$$

onde, \|.\| é a norma no $\mathbb{R}^{3}$ definida como:

$$
\|(X, Y, Z)\|=\sup \{|X|,|Y|+|Z|\}
$$

Da proposição 2.1.13, temos que, a órbita de $\gamma(t)$ permanece a uma distância positiva da fronteira de $\Omega$, assim, existe $0<k<1$ tal que: $1 \geq I_{v}(t) \geq k$ e $I_{h}(t) \geq k$.

Pelo fato de, $\gamma(t)$ ser uma órbita fechada, todos os pontos de $\gamma$ são $\omega$-limite. Assim, a função V está bem definida ao longo de $\gamma$ e

$$
\begin{aligned}
V\left(X, Y, Z, S_{h}, I_{h}, I_{v}\right) & =\left\|\left(X, \frac{I_{h}}{I_{v}} Y, \frac{I_{h}}{I_{v}} Z\right)\right\| \\
& =\sup \left\{|X|, \frac{I_{h}}{I_{v}}(|Y|+|Z|)\right\} \\
& \geq \sup \{|X|, k(|Y|+|Z|)\} .
\end{aligned}
$$

Portanto,

$$
V\left(X, Y, Z, S_{h}, I_{h}, I_{v}\right) \geq k\|(X, Y, Z)\| .
$$

Ao longo de $(X(t), Y(t), Z(t))$, soluções do sistema (2.22), temos:

$$
V(t)=\sup \left\{|X(t)|, \frac{I_{h}(t)}{I_{v}(t)}(|Y(t)|+|Z(t)|)\right\} .
$$

Pelo lema A.2.9 (consultar apêndice (A.2)) e, baseados no que foi desenvolvido em [LM95], obtemos as seguintes estimativas: 


$$
\begin{gathered}
D_{+}|X(t)| \leq-\left(\mu_{h}+a_{h} b \beta_{h} I_{v}+\gamma_{h}+\mu_{h}\right)|X(t)|+a_{h} b \beta_{h} S_{h}(|Y(t)|+|Z(t)|) \\
\leq-\left(\mu_{h}+a_{h} b \beta_{h} I_{v}+\gamma_{h}+\mu_{h}\right)|X(t)|+a_{h} b \beta_{h} S_{h} \frac{I_{v}}{I_{h}}\left(\frac{I_{h}}{I_{v}}(|Y(t)|+|Z(t)|)\right), \\
D_{+}|Y(t)| \leq a_{v} b \beta_{v}\left(1-I_{v}\right)|X(t)|-\left(\mu_{h}+a_{h} b \beta_{h} I_{v}+\mu_{v}+a_{v} b \beta_{v} I_{h}\right)|Y(t)|, \\
D_{+}|Z(t)| \leq a_{h} b \beta_{h} I_{v}|Y(t)|-\left(\mu_{v}+a_{v} b \beta_{v} I_{h}+\gamma_{h}+\mu_{h}\right)|Z(t)| .
\end{gathered}
$$

Então, somando as desigualdades (2.26) e (2.27) obteremos:

$$
D_{+}[|Y(t)|+|Z(t)|] \leq a_{v} b \beta_{v}\left(1-I_{v}\right)|X(t)|-\left(\mu_{v}+\mu_{h}+a_{v} b \beta_{v} I_{h}\right)(|Y(t)|+|Z(t)|),
$$

portanto,

$$
\begin{aligned}
D_{+}\left[\frac{I_{h}}{I_{v}}|Y(t)|+|Z(t)|\right] & =\left(\frac{I_{h}^{\prime} I_{v}-I_{v}^{\prime} I_{h}}{I_{v}^{2}}\right)(|Y(t)|+|Z(t)|)+\frac{I_{h}}{I_{v}} D_{+}[|Y(t)|+|Z(t)|] \\
& =\left(\frac{I_{h}^{\prime}}{I_{h}}-\frac{I_{v}^{\prime}}{I_{v}}\right) \frac{I_{h}}{I_{v}}(|Y(t)|+|Z(t)|)+\frac{I_{h}}{I_{v}} D_{+}[|Y(t)|+|Z(t)|] \\
& \leq \frac{I_{h}}{I_{v}} a_{v} b \beta_{v}\left(1-I_{v}\right)|X(t)|-\frac{I_{h}}{I_{v}}\left(\mu_{v}+\mu_{h}+a_{v} b \beta_{v} I_{h}\right)(|Y(t)|+|Z(t)|) \\
& +\frac{I_{h}}{I_{v}}\left(\frac{I_{h}^{\prime}}{I_{h}}-\frac{I_{v}^{\prime}}{I_{v}}\right) \frac{I_{h}}{I_{v}}(|Y(t)|+|Z(t)|) \\
& =\frac{I_{h}}{I_{v}} a_{v} b \beta_{v}\left(1-I_{v}\right)|X(t)|+\frac{I_{h}}{I_{v}}\left(\frac{I_{h}^{\prime}}{I_{h}}-\frac{I_{v}^{\prime}}{I_{v}}-\mu_{v}-\mu_{h}-a_{v} b \beta_{v} I_{h}\right)(|Y(t)|+|Z(t)|) .
\end{aligned}
$$

Das desigualdades, (2.25) e (2.28),segue:

$$
D_{+} V(t) \leq \sup \left\{h_{1}(t), h_{2}(t)\right\} V(t)
$$

onde,

$$
\begin{gathered}
h_{1}(t)=-\left(\mu_{h}+a_{h} b \beta_{h} I_{v}+\gamma_{h}+\mu_{h}\right)+a_{h} b \beta_{h} S_{h} \frac{I_{v}}{I_{h}} \\
h_{2}(t)=\frac{I_{h}}{I_{v}} a_{v} b \beta_{v}\left(1-I_{v}\right)+\frac{I_{h}^{\prime}}{I_{h}}-\frac{I_{v}^{\prime}}{I_{v}}-\mu_{h}-\mu_{v}-a_{v} b \beta_{v} I_{h} .
\end{gathered}
$$

Considere agora o sistema (2.3). Dividindo as equações de $I_{h}^{\prime}(t)$ e $I_{v}^{\prime}(t)$ por $I_{h}(t)$ e $I_{v}(t)$ respectivamente, podemos reescrevê-las como

$$
\begin{aligned}
& a_{h} b \beta_{h} S_{h} \frac{I_{v}}{I_{h}}=\frac{I_{h}^{\prime}}{I_{h}}+\gamma_{h}+\mu_{h}, \\
& a_{v} b \beta_{v}\left(1-I_{v}\right) \frac{I_{h}}{I_{v}}=\frac{I_{v}^{\prime}}{I_{v}}+\mu_{v} .
\end{aligned}
$$

Lembrando que $a_{h}=\frac{A / \mu_{v}}{N_{h}+m}$ e $a_{v}=\frac{N_{h}}{N_{h}+m}$, e usando as equações (2.30) e (2.31), temos

$$
\sup \left\{h_{1}(t), h_{2}(t)\right\} \leq-\mu_{h}+\frac{I_{h}^{\prime}}{I_{h}} .
$$


Assim, pela desigualdade (2.29) e pela Desigualdade de Gronwall A.2.10 (ver apêndice A.1),

$$
V(t) \leq V(0) I_{h} e^{-\mu_{h} t} \leq V(0) e^{-\mu_{h} t}
$$

o que implica que $V(t) \rightarrow 0$ quando $t \rightarrow \infty$.

Por fim, da desigualdade (2.24), segue que:

$$
(X(t), Y(t), Z(t)) \rightarrow 0 \quad \text { quando } \quad t \rightarrow \infty .
$$

Com isso, concluímos que o sistema (2.22) é assintoticamente estável e portanto a solução periódica $\gamma(t)$ é assintoticamente e orbitalmente estável. Isso conlui a prova da afirmação 2.1.15.

Logo, a prova destas afirmações conclui a prova do Teorema 2.1.10 e por conseguinte, este resultado garante que, se $R_{o}>1$, o equilíbrio endêmico, $E_{2}$ é globalmente assintoticamente estável.

Além de garantirmos as condições de estabilidade global dos pontos de equilíbrios, podemos determinar completamente a dinâmica do comportamento das soluções do sistema (2.3) quando $R_{o}>1$.

Teorema 2.1.16. Considere o sistema (2.3). Se $R_{o}>1$, a região $\Omega-\left\{\left(S_{h}, 0,0\right): 0 \leq S_{h} \leq 1\right\}$, é uma região assintoticamente estável para o equilibrio endemico. Além disso, todas as trajetórias começando no eixo $S_{h}$, aproximam-se do equilibrio livre de doença, $E_{1}$.

Prova: A primeira parte do teorema segue diretamente da transversalidade do campo de vetores do sistema (2.3) na região $\Omega-\left\{\left(S_{h}, 0,0\right): 0 \leq S_{h} \leq 1\right\}$ e pelo fato de que se $R_{o}>1$ o equilíbrio endêmico é globalmente assintoticamente estável. A última parte segue analogamente da proposição 2.1.13 .

\subsection{Modelo 2}

\subsubsection{Descrição do modelo matemático}

O modelo 2, cujo estudo será desenvolvido nesta subseção, segue a metodologia proposta por Esteva e Vargas, em [EV99]. Este modelo baseia-se na hipótese de que a população humana total é governada por uma função de crescimento exponencial. O diagrama de fluxo para este modelo é representado pela Figura 2.2 .

Sejam, $N_{h}$ e $N_{v}$ as populações totais de humanos e vetores, respectivamente. À população total humana é atribuído um crescimento exponencial, cuja equação diferencial é dada por:

$$
\frac{d N_{h}}{d t}=\left(\nu_{h}-\mu_{h}\right) N_{h} \quad, \quad N_{h}(0)=N_{h o},
$$

tais que, $\nu_{h}$ e $\mu_{h}$ correspondem as taxas de nascimento e morte de humanos, respectivamente, e $N_{h o}$ corresponde ao tamanho inicial da população total humana.

Este modelo assume uma taxa de morte causada pela doença, a qual é representada pelo parâmetro $\alpha_{h}$. No caso da dengue, estima-se que, a cada 50 milhões de casos, 10 mil resultem em óbitos ([EV99]). No entanto, assumiremos que as taxas de nascimento e recuperação de humanos, $\nu_{h}$ e $\gamma_{h}$, respectivamente, são maiores do que a taxa de morte associada à doença.

Os compartimentos $\bar{S}_{h}, \bar{I}_{h}, \bar{R}_{h}, \bar{S}_{v}$ e $\bar{I}_{v}$, correspondem as populações de humanos suscetíveis, humanos infectados, humanos recuperados, vetores suscetíveis e vetores infectados, respectivamente. Além disso, a população de vetores é mantida constante, com nascimentos e mortes dados pela taxa $\mu_{v}$. Os vetores nunca recuperam-se de uma infecç̧ão.

A taxa de contatos efetivos $\lambda_{h}$, corresponde ao número médio de contatos diários entre humanos suscetíveis e mosquitos infectados que resultarão em infecção se o mosquito que estiver picando um suscetível 


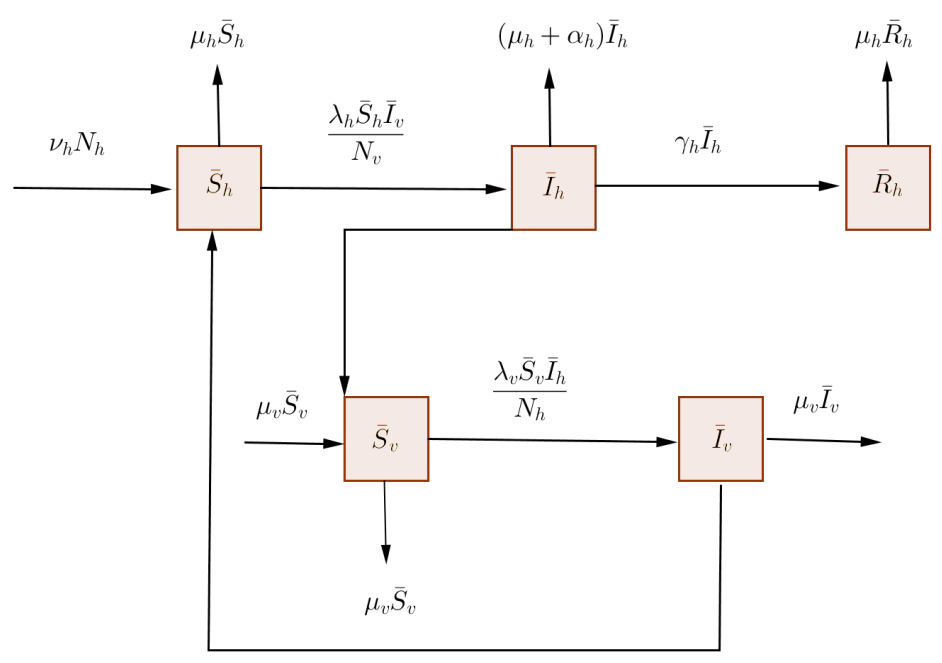

Figura 2.2: Dinâmica de propagação do dengue referente ao modelo 2

estiver infectado. A taxa de contatos efetivos $\lambda_{v}$, corresponde aos contatos efetivos entre um humano infectado e um vetor suscetível, isto é, o número médio de contatos diários adequados entre humanos infectados e vetores suscetíveis.

O modelo 2 assume como hipóteses:

- Somente um sorotipo do vírus da dengue é considerado;

- As populações de humanos e vetores são distribuídas homogeneamente;

- Todo vetor pode picar um humano com igual probabilidade;

- Os mosquitos nunca se recuperam de uma infecção;

- Humanos que recuperam-se adquirem imunidade permanente contra a doença;

- Este modelo não considera a transmissão vertical, isto é, todos os humanos recém-nascidos são suscetíveis;

Sob tais hipóteses, as equações diferenciais que descrevem o modelo 2 são dadas por:

$$
\left\{\begin{array}{l}
\frac{d \bar{S}_{h}}{d t}=\nu_{h} N_{h}-\mu_{h} \bar{S}_{h}-\lambda_{h} \bar{S}_{h} \bar{I}_{v} \\
\frac{d \bar{I}_{h}}{d t}=\lambda_{h} \bar{S}_{h} \bar{I}_{v} \\
\frac{\bar{I}_{v}}{N_{v}}-\left(\gamma_{h}+\mu_{h}+\alpha_{h}\right) \bar{I}_{h} \\
\frac{d \bar{R}_{h}}{d t}=\gamma_{h} \bar{I}_{h}-\nu_{h} \bar{R}_{h} \\
\frac{d \bar{I}_{v}}{d t}=\lambda_{v}\left(N_{v}-\bar{I}_{v}\right) \frac{\bar{I}_{h}}{N_{h}}-\mu_{v} \bar{I}_{v} \\
\frac{d N_{h}}{d t}=\left(\nu_{h}-\mu_{h}\right) N_{h}-\alpha_{h} \bar{I}_{h}
\end{array}\right.
$$

cujas condições iniciais são: $\bar{S}_{h}(0)=S_{h o} \geq 0, \bar{I}_{h}(0)=I_{h o} \geq 0, \bar{R}_{h}(0)=R_{h o} \geq 0, \bar{I}_{v}(0)=I_{v o} \geq 0$, $N_{h}(0)=N_{h o} \geq 0$. Todos os parâmetros do modelo acima são não negativos.

O sistema (2.32) é bem posto no sentido que, se as condições iniciais $\left(\bar{S}_{h}, \bar{I}_{h}, \bar{R}_{h}, \bar{I}_{v}, \bar{N}_{h}\right)$ estão em $\mathbb{R}_{+}^{5}$ então, todas as soluções do sistema (2.32) estarão definidas para todo $t \geq 0$ e permanecerão em $\mathbb{R}_{+}^{5}$. 
Sem perda de generalidade, podemos reescrever o sistema (2.32) introduzindo as proporções $S_{h}=\frac{\bar{S}_{h}}{N_{h}}$, $I_{h}=\frac{\bar{I}_{h}}{N_{h}}, R_{h}=\frac{\bar{R}_{h}}{N_{h}}, I_{v}=\frac{\bar{I}_{v}}{N_{v}}$ e usando a relação $R_{h}=1-S_{h}-I_{h}$, obtemos o seguinte sistema tridimensional, o qual descreve a dinâmica da proporções de indíviduos em cada uma das classes

$$
(M)\left\{\begin{array}{l}
S_{h}^{\prime}(t)=\nu_{h}\left(1-S_{h}\right)-\lambda_{h} S_{h} I_{v}+\alpha_{h} I_{h} S_{h} \\
I_{h}^{\prime}(t)=\lambda_{h} S_{h} I_{v}-m_{h} I_{h}+\alpha_{h} I_{h}^{2} \\
I_{v}^{\prime}(t)=\lambda_{v}\left(1-I_{v}\right) I_{h}-\mu_{v} I_{v}
\end{array}\right.
$$

tal que, $m_{h}=\gamma_{h}+\nu_{h}+\alpha_{h}$.

A região de interesse biológico

$$
\Omega=\left\{\left(S_{h}, I_{h}, I_{v}\right) \in \mathbb{R}^{3} / 0 \leq S_{h}, 0 \leq I_{h}, S_{h}+I_{h} \leq 1,0 \leq I_{h} \leq 1\right\}
$$

é positivamente invariante para o sistema (2.33). De fato,

i) Se $S_{h}=0$, então $S_{h}^{\prime}=\nu_{h} \geq 0$;

ii) Se $I_{h}=0$, então $I_{h}^{\prime}=\lambda_{h} S_{h} I_{v} \geq 0$;

iii) Se $I_{v}=0$, então $I_{v}^{\prime}=\lambda_{v} I_{h} \geq 0$;

iv) Se $I_{v}=1$, então $I_{v}^{\prime}=-\mu_{v} \leq 0$;

v) Se $S_{h}+I_{h}=1$, então $\left(S_{h}+I_{h}\right)^{\prime}=-\gamma_{h} I_{h} \leq 0$.

Observe que, apesar da população humana ser considerada variável neste modelo, as equações do sistema reduzido (2.33) não envolvem $N_{h}$ e portanto, podemos investigar o comportamento das proporções da população separadamente. Por outro lado, o tamanho das populações em cada uma das classes pode ser obtido resolvendo-se:

$$
N_{h}^{\prime}=\left(\nu_{h}-\mu_{h}-\alpha_{h} I_{h}\right) N_{h}
$$

\subsubsection{Pontos de equilíbrio e Número de Reprodutibilidade Basal}

Nossos primeiros resultados nesta subseção, concernem na existência e unicidade dos pontos de equilíbrio. Para isso, usaremos o parâmetro limiar, calculado conforme a metodologia apresentada em [DHR09]:

$$
R_{o}=\frac{\lambda_{h} \lambda_{v}}{\mu_{v} m_{h}}
$$

lembrando que, $m_{h}=\gamma_{h}+\nu_{h}+\alpha_{h}$.

Proposição 2.2.1. O sistema (2.33) tem um equiliırio livre de doença $E_{1}=(1,0,0)$. Se $R_{o}>1$, existe um único equilíbrio endemico $E_{2}=\left(S_{h}^{*}, I_{h}^{*}, I_{v}^{*}\right)$ no interior de $\Omega$.

Prova: A prova desta proposição consiste de duas etapas: 1) Vamos definir um intervalo que garanta a existência de $E_{2}$ no interior de $\Omega$ e, 2) Garantir a unicidade deste equilíbrio pelo Teorema do Valor Intermediário.

Da primeira e terceira equações do sistema (2.33), escrevendo $S_{h}$ e $I_{v}$ em função de $I_{h}$, tem-se que os pontos de equilíbrio devem obedecer as seguintes relações:

$$
\begin{gathered}
I_{v}=\frac{\lambda_{v} I_{h}}{\mu_{v}+\lambda_{v} I_{h}}, \\
S_{h}=\frac{\nu_{h}\left(\mu_{v}+\lambda_{v} I_{h}\right)}{\left(\nu_{h}-\alpha_{h} I_{h}\right)\left(\mu_{v}+\lambda_{v} I_{h}\right)+\lambda_{h} \lambda_{v} I_{h}} .
\end{gathered}
$$


Note que, fazendo $I_{h}=0$ e substituindo-o nas equações 2.35 e 2.36 vamos obter $S_{h}=1$ e $I_{v}=0$, o que nos dá o equilíbrio livre de doença, $E_{1}$. Suponha agora que $I_{h} \neq 0$. Substituindo as equações 2.35 e 2.36 na segunda equação do sistema (2.33), obteremos uma equação cúbica:

$$
\begin{aligned}
h\left(I_{h}\right) & =\lambda_{h} \lambda_{v} \nu_{h}-\left[m_{h}-\alpha_{h} I_{h}\right]\left[\left(\nu_{h}-\alpha_{h} I_{h}\right)\left(\mu_{v}+\lambda_{v} I_{h}\right)+\lambda_{h} \lambda_{v} I_{h}\right] \\
& =-\alpha_{h}^{2} \lambda_{v} I_{h}^{3}+\alpha_{h}\left(m_{h} \lambda_{v}+\nu_{h} \lambda_{v}-\alpha_{h} \mu_{v}+\lambda_{h} \lambda_{v}\right) I_{h}^{2} \\
& +\left(m_{h} \alpha_{h} \mu_{v}-m_{h} \lambda_{v} \lambda_{h}+\alpha_{h} \nu_{h} \mu_{v}-m_{h} \nu_{h} \lambda_{v}\right) I_{h}+\left(\lambda_{h} \lambda_{v} \nu_{h}-m_{h} \nu_{h} \mu_{v}\right) .
\end{aligned}
$$

Da equação (2.37), aplicando-se o limite e fazendo $I_{h}$ tender ao infinito, temos

$$
\begin{aligned}
& \lim _{I_{h} \rightarrow+\infty} h\left(I_{h}\right)=-\infty \\
& \lim _{I_{h} \rightarrow-\infty} h\left(I_{h}\right)=+\infty .
\end{aligned}
$$

Desta observação podemos concluir que a equação (2.37) tem pelo menos uma raiz real. Por outro lado, para mostramos que o equilíbrio endêmico está no interior de $\Omega$, ele deve satisfazer as seguintes desigualdades:

$$
\begin{gathered}
0<I_{h}<1, \\
0<\frac{\nu_{h}\left(\mu_{v}+\lambda_{v} I_{h}\right)}{\left(\nu_{h}-\alpha_{h} I_{h}\right)\left(\mu_{v}+\lambda_{v} I_{h}\right)+\lambda_{h} \lambda_{v} I_{h}}<1, \\
0<\frac{\lambda_{v} I_{h}}{\mu_{v}+\lambda_{v} I_{h}}<1, \\
0<S_{h}+I_{h}=1-\frac{\gamma_{h} I_{h}}{\nu_{h}-\alpha_{h} I_{h}}<1 .
\end{gathered}
$$

A desigualdade 2.40 segue diretamente de 2.38. No caso de 2.39, a desigualdade é verdadeira se, verificadas as seguintes condições:

i) $\nu_{h}>\alpha_{h}$;

ii) $0<I_{h}<1$;

iii) $I_{h}<\left(\frac{\lambda_{h} \lambda_{v}}{\alpha_{h} \mu_{v}}-1\right) \frac{\mu_{v}}{\lambda_{v}}$.

As condições $i$ e $i$ i são satisfeitas, pois são hipóteses do modelo matemático. A condição $i i i$ também é verificada. De fato, para que a desigualdade 2.39 seja satisfeita precisamos que:

a) $\nu_{h}\left(\mu_{v}+\lambda_{v} I_{h}\right)>0$;

b) $\nu_{h}\left(\mu_{v}+\lambda_{v} I_{h}\right)<\left(\nu_{h}-\alpha_{h} I_{h}\right)\left(\mu_{v}+\lambda_{v} I_{h}\right)+\lambda_{h} \lambda_{v} I_{h}$.

O item $a$ ) é trivialmente satisfeito pois, $I_{h}>0$ e além disso todos os parâmetros do modelo são negativos. Para o item $b$ ), temos por hipótese que $\nu_{h}>\alpha_{h}$ então, se dividirmos ambos os lados de $b$ ) por $\mu_{v}+\lambda_{v} I_{h}$, após algumas manipulações algébricas obtemos:

$$
I_{h}<\left(\frac{\lambda_{h} \lambda_{v}}{\alpha_{h} \mu_{v}}-1\right) \frac{\mu_{v}}{\lambda_{v}}
$$

e então, a desigualdade (2.40) também é satisfeita.

Finalmente, verifica-se a desigualdade $2.41 \mathrm{se}, 0<I_{h}<1$ e $I_{h}<\frac{\nu_{h}}{\gamma_{h}+\alpha_{h}}$. Portanto, para garantirmos que, as raízes de (2.37) estão no interior de $\Omega$, isto é, que o equilíbrio endêmico está no interior de $\Omega$, devemos observar as raízes de $h\left(I_{h}\right)$ no intervalo $\left(0, I_{h \max }\right)$, cujo

$$
I_{h \max }=\min \left\{1,\left(\frac{\lambda_{h} \lambda_{v}}{\alpha_{h} \mu_{v}}-1\right) \frac{\mu_{v}}{\lambda_{v}}, \frac{\nu_{h}}{\gamma_{h}+\alpha_{h}}\right\} .
$$


Observe que, pelo fato de $S_{h}$ e $I_{v}$ serem dados em função de $I_{h}$, se garantirmos que as soluções da equação (2.37) estão no interior de $\Omega$, então $S_{h}$ e $I_{v}$ também estarão no interior de $\Omega$. Além disso, precisamos verificar se $I_{h \max }$ está à esquerda ou à direita do intervalo pois, como $I_{h \max }$ é definido como o valor mínimo do conjunto, esta conclusão não é óbvia.

De fato, para $1 \mathrm{e} \frac{\nu_{h}}{\gamma_{h}+\alpha_{h}}, I_{h \max }$ é positivo. Por outro lado, para analisarmos $\left(\frac{\lambda_{h} \lambda_{v}}{\alpha_{h} \mu_{v}}-1\right) \frac{\mu_{v}}{\lambda_{v}}$ precisamos dividir a análise em dois casos:

Caso i) Se $\frac{\lambda_{h} \lambda_{v}}{\alpha_{h} \mu_{v}} \leq 1$, então $I_{h m a x}$ é não-positivo (poderia ser zero se $\frac{\lambda_{h} \lambda_{v}}{\alpha_{h} \mu_{v}}=1$ ) e neste caso o valor mínimo de $I_{h m a x}$ seria dado por $\left(\frac{\lambda_{h} \lambda_{v}}{\alpha_{h} \mu_{v}}-1\right) \frac{\mu_{v}}{\lambda_{v}}$. Isso implicaria que as raízes de (2.37), estariam definidas para um intervalo negativo, o que não faria sentido epidemiologicamente e portanto, concluiríamos que o único equilíbrio do sistema (2.33), no interior de $\Omega$ é o equilíbrio livre de doença. Observe que, este é um caso especial de $R_{o}<1$.

Caso ii) Suponha que $\frac{\lambda_{h} \lambda_{v}}{\alpha_{h} \mu_{v}}>1$. Neste caso, $I_{h \max }>0$ e portanto, $h\left(I_{h \max }\right)<0$.

De fato, vamos supor inicialmente que $I_{h \max }=1$. Substituindo $I_{h \max }=1$ na equação (2.37), obtemos:

$$
h(1)=\nu_{h} \lambda_{h} \lambda_{v}-\left[m_{h}-\alpha_{h}\right]\left[\left(\nu_{h}-\alpha_{h}\right)\left(\mu_{v}+\lambda_{v}\right)+\lambda_{h} \lambda_{v}\right] .
$$

Substituindo, $m_{h}=\gamma_{h}+\nu_{h}+\alpha_{h}$ na expressão acima, usando o fato que, $\nu_{h}>\alpha_{h}$ e que todos os parâmetros do modelo são não negativos, após algumas manipulações algébricas é possível verificar

$$
h(1)=\nu_{h} \lambda_{h} \lambda_{v}-\left[\gamma_{h}\left(\nu_{h}-\alpha_{h}\right)\left(\mu_{v}+\lambda_{v}\right)+\gamma_{h} \lambda_{h} \lambda_{v}+\nu_{h}\left(\nu_{h}-\alpha_{h}\right)\left(\mu_{v}+\lambda_{v}\right)+\nu_{h} \lambda_{h} \lambda_{v}\right]<0 .
$$

Da mesma forma, suponha que $I_{h \max }=\frac{\nu_{h}}{\gamma_{h}+\alpha_{h}}$. Analogamente, substituindo $I_{h \max }$ na equação (2.37) e usando os mesmos argumentos do caso anterior, temos:

$$
h\left(\frac{\nu_{h}}{\gamma_{h}+\alpha_{h}}\right)<0
$$

Finalmente, para $I_{h \max }=\left(\frac{\lambda_{h} \lambda_{v}}{\alpha_{h} \mu_{v}}-1\right) \frac{\mu_{v}}{\lambda_{v}}$. Substituindo o valor de $I_{h \max }$ na equação de $h\left(I_{h \max }\right)$ e sob as hipóteses:

1) $\nu_{h}>\alpha_{h}$

2) $\frac{\lambda_{h} \lambda_{v}}{\alpha_{h} \mu_{v}}>1$;

3) $0<\left(\frac{\lambda_{h}}{\alpha_{h}}-\frac{\mu_{v}}{\lambda_{v}}\right)<1$;

4) $0<\left(\frac{\lambda_{h}}{\alpha_{h}}-\frac{\mu_{v}}{\lambda_{v}}\right)<\frac{\nu_{v}}{\gamma_{h}+\alpha_{h}}$;

é possível mostrar, após algumas manipulações algébricas que, $h\left(\left(\frac{\lambda_{h} \lambda_{v}}{\alpha_{h} \mu_{v}}-1\right) \frac{\mu_{v}}{\lambda_{v}}\right)<0$ e portanto, $h\left(I_{\text {max }}\right)<0$.

Verificaremos agora, quais são os pontos críticos da equação (2.37). Fazendo $h^{\prime}\left(I_{h}\right)=0$, obtemos como raízes:

$$
\begin{aligned}
& I_{h 1}=\frac{\left(\lambda_{v} \nu_{h}+\lambda_{h} \lambda_{v}-\alpha_{h} \mu_{v}+\lambda_{v} m_{h}\right)-\sqrt{\Delta}}{3 \alpha_{h} \lambda_{v}} \\
& I_{h 2}=\frac{\left(\lambda_{v} \nu_{h}+\lambda_{h} \lambda_{v}-\alpha_{h} \mu_{v}+\lambda_{v} m_{h}\right)+\sqrt{\Delta}}{3 \alpha_{h} \lambda_{v}}
\end{aligned}
$$


tais que, $\Delta=\left(\lambda_{v} \nu_{h}+\lambda_{h} \lambda_{v}-\alpha_{h} \mu_{v}+\lambda_{v} m_{h}\right)^{2}+3 \lambda_{v}\left(\alpha_{h} \nu_{h} \mu_{v}-\left(\lambda_{v} \nu_{h}+\lambda_{h} \lambda_{v}-\alpha_{h} \mu_{v}\right) m_{h}\right)>\lambda_{v} \nu_{h}$.

Das expressões (2.42) e (2.43), obtemos que $I_{h 2}$ é um máximo local de $h\left(I_{h}\right)$ pois, $h\left(I_{h 2}\right)^{\prime \prime}<0$. Logo,

$$
\begin{aligned}
I_{h 2} & >\frac{\lambda_{v} \nu_{h}+\lambda_{h} \lambda_{v}-\alpha_{h} \mu_{v}+\lambda_{v} m_{h}+\lambda_{v} \nu_{h}}{3 \alpha_{h} \lambda_{v}} \\
& >\frac{\nu_{h}}{\alpha_{h}} \\
& \geq I_{\text {hmax }} .
\end{aligned}
$$

Note que, a desigualdade acima é verdadeira para cada um dos elementos de $I_{h m a x}$ pois, $\nu_{h}>\alpha_{h}$ implica que, $\frac{\nu_{h}}{\alpha_{h}}>1$ e $\frac{\nu_{h}}{\alpha_{h}}>\frac{\nu_{h}}{\alpha_{h}+\gamma_{h}}$. Por fim, as duas últimas condições juntas implicam que $\frac{\nu_{h}}{\alpha_{h}}>$ $\left(\frac{\lambda_{h} \lambda_{v}}{\alpha_{h} \mu_{v}}-1\right) \frac{\mu_{v}}{\lambda_{v}}$.

Com isso, concluímos que o máximo local da equação 2.37 encontra-se do lado direito do intervalo $\left[0, I_{h \max }\right]$ e, uma vez que, $h\left(I_{h \max }\right)<0$ temos pelo Teorema do Valor Intermediário que: se $h(0)>0$ e $h\left(I_{h \max }\right)<0$, a equação $(2.37)$ tem uma única raiz $I_{h}^{*}$ em $\left(0, I_{h \max }\right)$.

De fato, $h(0)>0$ se e somente se, $R_{o}>1$ pois,

$$
h(0)=\nu_{h}\left(\lambda_{h} \lambda_{v}-\mu_{v} m_{h}\right)=\frac{\nu_{h}}{\mu_{v} m_{h}}\left(\frac{\lambda_{h} \lambda_{v}}{\mu_{v} m_{h}}-1\right)=\frac{\nu_{h}}{\mu_{v} m_{h}}\left(R_{o}-1\right) .
$$

Isso conclui a prova da proposição.

Com este resultado, verificamos que, se $R_{o}<1$, o sistema (2.33) tem um único equilíbrio livre de doença no interior de $\Omega$ e se, $R_{o}>1$, o sistema (2.33) tem um único equilíbrio endêmico, $E_{2}$, no interior de $\Omega$. Garantidas, a existência e unicidade dos equilíbrios, nosso próximo passo consiste em, estabelecer condições, que garantam a estabilidade local e global destes equilíbrios.

\subsubsection{Análise de estabilidade dos pontos de equilíbrio}

A análise de estabilidade dos pontos de equilíbrio do modelo 2 seguirá a mesma ordem apresentada na Subseção 2.1.3, seguindo a metodologia proposta por Esteva e Vargas, em [EV99]. Essencialmente, usaremos os mesmos argumentos matemáticos para demonstrar a estabilidade local e global dos equilíbrios.

Para mostrarmos a estabilidade global de $E_{1}$, mostraremos que o sistema (2.33) é persistente no sentido descrito em [BFW86].

Proposição 2.2.2. No bordo de $\Omega$ o sistema (2.33) tem somente um $\omega$-limite que é o equilibrio livre de doença, $E_{1}$. Além disso, para $R_{o}>1, E_{1}$ não pode ser $\omega$-limite de qualquer órbita no interior de $\Omega$.

Prova: Observe que, o campo de vetores associado ao fluxo do sistema (2.33) é transversal no bordo de $\Omega$, ou seja, no bordo de $\Omega$ todas as direções do campo apontam para o interior de $\Omega$, exceto, o eixo $S_{h}$ que é invariante pelo fluxo de M (M define o sistema (2.33)) pois, $M\left(S_{h}, 0,0\right) \subset\left(S_{h}, 0,0\right)$ e além disso, no eixo $S_{h}$ temos $S_{h}^{\prime}=\nu_{h}\left(1-S_{h}\right)$, cujas órbitas do sistema (2.33), restrito ao eixo $S_{h}$, são: $\left(S_{h}, 0,0\right)$ em que $S_{h}<1$, $\left(S_{h}, 0,0\right)$ em que $S_{h}>1$ e $(1,0,0)$ que é o equilíbrio assintoticamente estável. Portanto, $S_{h} \rightarrow 1$ quando $t \rightarrow \infty$, isso implica que, $E_{1}$ é o único $\omega$-limite no bordo de $\Omega$.

Para provarmos a segunda parte da proposição considere a função

$$
L(t)=I_{v}(t)+\frac{\mu_{v}\left(1+R_{o}\right)}{2 \lambda_{h}} I_{h}(t)
$$

Derivando L ao longo das soluções, tem-se 


$$
\begin{aligned}
L^{\prime} & =I_{v}^{\prime}+\frac{\mu_{v}\left(1+R_{o}\right)}{2 \lambda_{h}} I_{h}^{\prime} \\
& =\left[\lambda_{v}\left(1-I_{v}\right) I_{h}-\mu_{v} I_{v}\right]+\frac{\mu_{v}\left(1+R_{o}\right)}{2 \lambda_{h}}\left[\lambda_{h} S_{h} I_{v}-m_{h} I_{h}+\alpha_{h} I_{h}^{2}\right] \\
& =\left[\frac{\mu_{v}\left(1+R_{o}\right)}{2} S_{h}-\mu_{v}\right] I_{v}+\left[\lambda_{v}\left(1-I_{v}\right)-\frac{\mu_{v} m_{h}\left(1+R_{o}\right)}{2 \lambda_{h}}+\frac{\mu_{v} \alpha_{h}\left(1+R_{o}\right)}{2 \lambda_{h}} I_{h}\right] I_{h} \\
& =\left[S_{h}-\frac{2}{1+R_{o}}\right] \frac{\mu_{v}\left(1+R_{o}\right)}{2} I_{v}+\left[\lambda_{v}\left(\left(1-I_{v}\right)-\frac{1}{2}\left(\frac{1}{R_{o}}+1\right)\right)+\frac{\mu_{v} \alpha_{h}}{2 \lambda_{h}}\left(1+R_{o}\right) I_{h}\right] I_{h} .
\end{aligned}
$$

Note que, para $R_{o}>1$, temos: $\frac{2}{1+R_{o}}<1$ e $\frac{1}{2}\left(\frac{1}{R_{o}}+1\right)<1$. Sendo assim, existe uma vizinhança $U$ de $E_{1}$, tal que, $\left(S_{h}, I_{h}, I_{v}\right) \in U \cap \operatorname{int}(\Omega)$, onde as expressões entre colchetes são positivas. Isso implica que, nessa vizinhança $U, V^{\prime}>0$, exceto em $(1,0,0)$.

Ou seja, as curvas de níveis de L,

$$
I_{v}(t)+\frac{\mu_{v}\left(1+R_{o}\right)}{2 \lambda_{h}} I_{h}(t)=c,
$$

se afastam do eixo $S_{h}$ conforme $c$ cresce. Assim, uma vez que L cresce ao longo das órbitas, começando em $U \cap \operatorname{int}(\Omega)$, podemos concluir que as órbitas se afastam de $E_{1}$ e portanto, para $R_{o}>1, E_{1}$ não pode ser $\omega$-limite de qualquer órbita no int $(\Omega)$. Com isso, provamos que o sistema (2.33) é persistente.

Munidos da Proposição 2.2.2, mostraremos que o equilíbrio livre de doença é globalmente assintoticamente estável. Todavia, diferentemente dos argumentos usados até então, para garantir a estabilidade global de $E_{1}$, usaremos uma propriedade dos sistemas competitivos tridimensionais.

Proposição 2.2.3. O sistema (2.33) é competitivo.

Proposição 2.2.4. O equilibrio $E_{1}$ é globalmente assintoticamente estável em $\Omega$ se $R_{o} \leq 1$ e instável se $R_{o}>1$.

Prova: Suponha que $\Gamma$ é uma órbita periódica não trivial, contida em $\Omega$. Pela Proposição 2.2.2, $\Gamma$ não está no bordo de $\Omega$ pois, o $\omega$-limite de uma órbita periódica, é a própria órbita e, pela Proposição 2.2 .2 foi mostrado que o único ponto $\omega$-limite no bordo de $\Omega$ é $E_{1}$.

Além disso, o fato do eixo $S_{h}$ ser positivamente invariante implica que, a intersecção entre $\Gamma$ e o eixo $S_{h}$ seja vazia. Contudo, como supomos que existe uma curva $\Gamma$ e ela não pode estar no bordo de $\Omega$ e nem interceptar o eixo $S_{h}$, vai existir $\varepsilon>0$ tal que:

$$
\Gamma \subset[\bar{a}, \bar{b}] \subset \Delta
$$

cujos, $\bar{a}=(0, \varepsilon, \varepsilon)$ e $\bar{b}=(1,1,1)$ e $\Delta$ é o cubo unitário.

Dado que, o cubo unitário é convexo e que o sistema (2.33) é competitivo em $\Omega$, note que a região $[\bar{a}, \bar{b}]$ que contém $\Gamma$, está definida de forma que $E_{1} \notin[\bar{a}, \bar{b}]$, mas esteja contido no cubo unitário. De fato, $E_{1} \notin[\bar{a}, \bar{b}]$, pois concluímos anteriormente que a interseç̧ão entre a curva $\Gamma$ e o eixo $S_{h}$ é vazia logo, como $E_{1}=(1,0,0)$ pertence ao eixo $S_{h}$ então $E_{1}$ não pode pertencer a região $[\bar{a}, \bar{b}]$.

Assim, pelo teorema A.2.6 (consultar Apêndice A.2), $[\bar{a}, \bar{b}]$ deve conter um ponto de equilíbrio. Por outro lado, pela Proposição 2.2.2, para $R_{o} \leq 1, E_{1}$ é o único equilíbrio em $\Delta$ e como vimos, ele não está em $[\bar{a}, \bar{b}]$, sendo assim, não podemos ter soluções do tipo periódicas em $\Omega$ e portanto a estabilidade global de $E_{1}$ segue da propriedade de Poincaré Bendixson para sistemas competitivos ([ZS94], [Hir90]). Isso conclui a primeira parte da proposição.

Para a segunda parte, precisaremos calcular os autovalores do sistema (2.33) linearizado em torno do equilíbrio livre de doença e mostrar que para $R_{o}>1$, este equilíbrio é instável. 
$\operatorname{Seja} \operatorname{DM}\left(E_{1}\right)$, a diferencial do campo M aplicada ao equilíbrio $E_{1}$ :

$$
D M\left(E_{1}\right)=\left[\begin{array}{ccc}
-\nu_{h} & \alpha_{h} & -\gamma_{h} \\
0 & -m_{h} & \gamma_{h} \\
0 & \lambda_{v} & -\mu_{v}
\end{array}\right] .
$$

Calculando o polinômio característico $P(\Lambda)$ de $\operatorname{DM}\left(E_{1}\right)$, temos:

$$
P(\Lambda)=-\left(\nu_{h}+\Lambda\right)\left[\Lambda^{2}+\left(m_{h}+\mu_{v}\right) \Lambda-m_{h} \mu_{v}\left(R_{o}-1\right)\right]
$$

De imediato temos uma das raízes $\Lambda_{1}=-\nu_{v}<0$. Por outro lado, analisando o polinômio de grau 2 e aplicando o critério de Routh-Hurwitz temos que o equilíbrio $E_{1}$ é instável para $R_{o}>1$ e isso conclui a prova da proposição.

Verificadas a estabilidade local e global do equilíbrio livre de doença, nosso próximo resultado consiste na prova da estabilidade local do equilíbrio endêmico, $E_{2}$.

Proposição 2.2.5. O equilißrio endemico, $E_{2}$, é localmente assintoticamente estável em $\Omega$ se $R_{o}>1$.

Prova: A estabilidade local do equilíbrio endemico será determinada através do critério de Routh-Hurwitz. A partir do sistema (2.33) avaliado em $E_{2}=\left(S_{h}^{*}, I_{h}^{*}, I_{v}^{*}\right)$, vamos extrair algumas relações. Isolando-se $S_{h}$, da primeira e segunda equações de (2.33), obtemos:

$$
\begin{gathered}
S_{h}^{*}=\frac{a b}{\lambda_{h} \lambda_{v}}, \\
\frac{-\nu_{h}}{S_{h}^{*}}=-\nu_{h}-\lambda_{h} I_{v}^{*}+\alpha_{h} I_{h}^{*}
\end{gathered}
$$

tais que, $a=\left(\mu_{v}+\lambda_{v} I_{h}^{*}\right)$ e $b=\left(m_{h}-\alpha I_{h}^{*}\right)$.

Considere a linearização do sistema (2.33), avaliado no ponto $E_{2}$. Substituindo as relações (2.44) e (2.45), a matriz jacobiana do sistema (2.33) aplicada ao equilíbrio $E_{2}$, pode ser escrita como:

$$
D M\left(E_{2}\right)=\left[\begin{array}{ccc}
-\frac{\lambda_{h} \lambda_{v} \nu_{h}}{a b} & \frac{\alpha_{h} a b}{\lambda_{h} \lambda_{v}} & -\frac{a b}{\lambda_{v}} \\
-\frac{\lambda_{h} \lambda_{v} I_{h}^{*}}{a b} & -b+\alpha_{h} I_{h}^{*} & \frac{a b}{\lambda_{v}} \\
0 & \frac{\lambda_{v} \mu_{v}}{a} & -a
\end{array}\right]
$$

Calculando o polinômio característico associado a matriz (2.46), é possível obter:

$$
P_{1}(\Lambda)=a_{o} \Lambda^{3}+a_{1} \Lambda^{2}+a_{2} \Lambda+a_{3},
$$

onde:

$$
\begin{aligned}
& a_{o}=1 \\
& a_{1}=\frac{\lambda_{h} \lambda_{v} \nu_{h}}{a b}+a+b-\alpha_{h} I_{h}^{*} \\
& a_{2}=\left(b-\alpha_{h} I_{h}^{*}\right) \lambda_{v} I_{h}^{*}+\frac{\lambda_{h} \lambda_{v} \nu_{h}}{b}+\frac{\lambda_{h} \lambda_{v} \nu_{h}}{a b}\left(b-\alpha_{h} I_{h}^{*}\right)-b \alpha_{h} I_{h}^{*}-\mu_{v} \alpha_{h} I_{h}^{*} \\
& a_{3}=\left(b-\alpha_{h} I_{h}^{*}\right) \frac{\lambda_{h} \lambda_{v} \nu_{h}}{b}-\frac{\lambda_{h} \lambda_{v} \mu_{v}}{a}\left(\nu_{h}-b I_{h}^{*}\right)-\alpha_{h} a b I_{h}^{*} .
\end{aligned}
$$


Queremos concluir, pelo teorema de Routh Hurwitz que, $E_{2}$ é localmente assintoticamente estável. Para isso, precisamos verificar se, $a_{o}, a_{1}, a_{2}, a_{3}>0$ e ainda, $a_{1} a_{2}>a_{3} a_{o}$.

Note que, ao combinarmos as duas hipóteses, $\nu_{h}>\alpha_{h}$ e $\gamma_{h}>\alpha_{h}$, vamos ter que, $b-\alpha_{h} I_{h}^{*}>\alpha_{h}$ e por conseguinte $a_{1}>0$.

Somando-se as duas primeiras equações do sistema (2.33) e observando que, $S_{h}+I_{h}=1$ quando aplicadas ao equilíbrio endemico $E_{2}$, é possível obter após algumas manipulações algébricas a seguinte expressão:

$$
b I_{h}^{*}=\nu_{h}-\left(\nu_{h}-\alpha_{h} I_{h}^{*}\right) S_{h}^{*}
$$

que por sua vez, implica em:

$$
b I_{h}^{*}=\nu_{h}-\left(\nu_{h}-\alpha_{h} I_{h}^{*}\right) S_{h}^{*}<\nu_{h} .
$$

Além disso, uma vez que $S_{h}^{*}<1$, podemos concluir a partir da equação (2.44) que:

$$
\lambda_{h} \lambda_{v}>a b
$$

Portanto, de $b-\alpha_{h} I_{h}^{*}>\alpha_{h}$ e das relações (2.48) e (2.49), temos:

$$
\begin{aligned}
a_{2} & =\left(b-\alpha_{h} I_{h}^{*}\right) \lambda_{v} I_{h}^{*}+\frac{\lambda_{h} \lambda_{v} \nu_{h}}{b}+\frac{\lambda_{h} \lambda_{v} \nu_{h}}{a b}\left(b-\alpha_{h} I_{h}^{*}\right)-b \alpha_{h} I_{h}^{*}-\mu_{v} \alpha_{h} I_{h}^{*} \\
& >\left(b-\alpha_{h} I_{h}^{*}\right) \lambda_{v} I_{h}^{*}+\frac{a \nu_{h} \lambda_{h} \lambda_{v}+\alpha_{h} \nu_{h} \lambda_{h} \lambda_{v}}{a b}-b \alpha_{h} I_{h}^{*}-\mu_{v} \alpha_{h} I_{h}^{*} \\
& =\left(b-\alpha_{h} I_{h}^{*}\right) \lambda_{v} I_{h}^{*}+\left(a \nu_{h}+\alpha_{h} \nu_{h}\right) \frac{\lambda_{h} \lambda_{v}}{a b}-b \alpha_{h} I_{h}^{*}-\mu_{v} \alpha_{h} I_{h}^{*} \\
& >\left(b-\alpha_{h} I_{h}^{*}\right) \lambda_{v} I_{h}^{*}+a \nu_{h}+\alpha_{h} \nu_{h}-b \alpha_{h} I_{h}^{*}-\mu_{v} \alpha_{h} I_{h}^{*} .
\end{aligned}
$$

Como $b I_{h}^{*}<\nu_{h}$ temos $\alpha_{h} \nu_{h}-b \alpha_{h} I_{h}^{*}>0$ e, $\nu_{h}-\alpha_{h} I_{h}^{*}$, implica $a \nu_{h}-\mu_{v} \alpha_{h} I_{h}^{*}$, podemos concluir que $a_{2}>0$.

Para $a_{3}$, usando a equação (2.45) e a relação (2.48) tem-se:

$$
\begin{aligned}
a_{3} & =\left(b-\alpha_{h} I_{h}^{*}\right) \frac{\lambda_{h} \lambda_{v} \nu_{h}}{b}-\frac{\lambda_{h} \lambda_{v} \mu_{v}}{a}\left(\nu_{h}-b I_{h}^{*}\right)-\alpha_{h} a b I_{h}^{*} \\
& =\left(b-\alpha_{h} I_{h}^{*}\right) \frac{\lambda_{h} \lambda_{v} \nu_{h}}{b}-b \mu_{v}\left(\nu_{h}-b I_{h}^{*}\right)-\alpha_{h} a b I_{h}^{*} \\
& =\lambda_{h} \lambda_{v} \nu_{h}-\frac{\lambda_{h} \lambda_{v} \nu_{h} \alpha_{h} I_{h}^{*}}{b}-b \mu_{v}\left(\nu_{h}-b I_{h}^{*}\right)-\alpha_{h} a b I_{h}^{*}
\end{aligned}
$$

Da equação (2.37) aplicada ao equilíbrio endemico, ou seja, $h\left(I_{h}^{*}\right)=0$ temos que $\left(\lambda_{h} \lambda_{v} \nu_{h}\right)$ é dado por:

$$
\begin{aligned}
\lambda_{h} \lambda_{v} \nu_{h} & =\left[m_{h}-\alpha_{h} I_{h}^{*}\right]\left[\left(\nu_{h}-\alpha_{h} I_{h}^{*}\right)\left(\mu_{v}+\lambda_{v} I_{h}^{*}\right)+\lambda_{h} \lambda_{v} I_{h}^{*}\right] \\
& =b\left(\nu_{h}-\alpha_{h} I_{h}^{*}\right)\left(\mu_{v}+\lambda_{v} I_{h}^{*}\right)+b \lambda_{h} \lambda_{v} I_{h}^{*} \\
& =b \mu_{v}\left(\nu_{h}-\alpha_{h} I_{h}^{*}\right)+b\left(\nu_{h}-\alpha_{h} I_{h}^{*}\right) \lambda_{v} I_{h}^{*}+b \lambda_{h} \lambda_{v} I_{h}^{*} .
\end{aligned}
$$

Substituindo, a expressão de $\left(\lambda_{h} \lambda_{v} \nu_{h}\right)$ na expressão de $a_{3}$, vamos ter:

$$
\begin{aligned}
a_{3} & =b\left(\nu_{h}-\alpha_{h} I_{h}^{*}\right) \lambda_{v} I_{h}^{*}+b \lambda_{h} \lambda_{v} I_{h}^{*}-\frac{\lambda_{h} \lambda_{v} \nu_{h} \alpha_{h} I_{h}^{*}}{b}-\alpha_{h} a b I_{h}^{*} \\
& >\left(\nu_{h}-\alpha_{h} I_{h}^{*}\right) \lambda_{v} I_{h}^{*}+\lambda_{h} \lambda_{v}\left(b-2 \alpha_{h}\right) I_{h}^{*} \\
& >0 .
\end{aligned}
$$


Com isso, mostramos que a primeira condição do critério de Routh Hurwitz é satisfeita. Resta-nos mostrar a segunda condição. De fato,

$$
\begin{aligned}
a_{1} a_{2} & =\left[\frac{\lambda_{h} \lambda_{v} \nu_{h}}{a b}+a+b-\alpha_{h} I_{h}^{*}\right]\left[\left(b-\alpha_{h} I_{h}^{*}\right) \lambda_{v} I_{h}^{*}+\frac{\lambda_{h} \lambda_{v} \nu_{h}}{b}+\frac{\lambda_{h} \lambda_{v} \nu_{h}}{a b}\left(b-\alpha_{h} I_{h}^{*}\right)-b \alpha_{h} I_{h}^{*}-\mu_{v} \alpha_{h} I_{h}^{*}\right] \\
& >a\left[\frac{\lambda_{h} \lambda_{v} \nu_{h}}{a b}\left(b-\alpha_{h} I_{h}^{*}\right)-b \alpha_{h} I_{h}^{*}\right] \\
& =\frac{\lambda_{h} \lambda_{v} \nu_{h}}{b}\left(b-\alpha_{h} I_{h}^{*}\right)-\alpha_{h} I_{h}^{*} \\
& >\frac{\lambda_{h} \lambda_{v} \nu_{h}}{b}\left(b-\alpha_{h} I_{h}^{*}\right)-\frac{\lambda_{h} \lambda_{v} \mu_{v}}{a}\left(\nu_{h}-b I_{h}^{*}\right)-\alpha_{h} a b I_{h}^{*} \\
& =a_{3} a_{1}
\end{aligned}
$$

Portanto, pelo critério de Routh Hurwitz, temos que o equilíbrio endemico $E_{2}$ é localmente assintoticamente estável para $R_{o}>1$.

Até o momento verificamos a estabilidade global de $E_{1}$ e estabilidade local de $E_{2}$. Mostramos que o sistema (2.33) é competitivo e persistente. Conforme o Teorema 2.1.10, resta-nos verificar que o sistema (2.33) possui a propriedade da estabilidade de órbitas periódicas e então concluiremos que se $R_{o}>1 E_{2}$ é globalmente estável. A prova deste resultado utilizará os mesmos argumentos da Subseção 2.1.3.

Teorema 2.2.6. Para $R_{o}>1$ o equilibrio endemico $E_{2}$ é globalmente assintoticamente estável em $\Omega-\left\{\left(S_{h}, 0,0\right): 0 \leq S_{h} \leq 1\right\}$.

Prova: Da transversalidade do campo de vetores associado ao sistema $(2.33)$, em $\Omega-\left\{\left(S_{h}, 0,0\right): 0 \leq S_{h} \leq 1\right\}$, e pela invariância do eixo $S_{h}$, precisamos mostrar que $E_{2}$ é globalmente assintoticamente estável no interior de $\Omega$.

Para o modelo 2, a equação de segunda composição aditiva é dada pelo sistema:

$$
\left\{\begin{array}{l}
X^{\prime}=-\left(\lambda_{h} I_{v}+\nu_{h}-\alpha_{h} I_{h}+m_{h}-2 \alpha_{h} I_{h}\right) X+\lambda_{h} S_{h} Y+\lambda_{h} S_{h} Z \\
Y^{\prime}=\lambda_{v}\left(1-I_{v}\right) X-\left(\lambda_{v} I_{v}+\nu_{h}-\alpha_{h} I_{h}+\lambda_{v} I_{h}+\mu_{v}\right) Y+\alpha_{h} S_{h} Z \\
Z^{\prime}=\lambda_{h} I_{v} Y-\left(m_{h}-2 \alpha_{h} I_{h}+\lambda_{v} I_{h}+\mu_{v}\right) Z .
\end{array}\right.
$$

Considere a seguinte função de Lyapunov,

$$
V\left(X(t), Y(t), Z(t), S_{h}(t), I_{h}(t), I_{v}(t)\right)=\left\|\left(X(t), \frac{I_{h}(t)}{I_{v}(t)} Y(t), \frac{I_{h}(t)}{I_{v}(t)} Z(t)\right)\right\|
$$

tal que, $\|$.$\| é a norma no \mathbb{R}^{3}$, definida como:

$$
\|(X, Y, Z)\|=\sup \{|X|,|Y|+|Z|\}
$$

Fazendo a derivada positiva de $|X(t)|,|Y(t)|$ e $|Z(t)|$ é possível obter algumas estimativas:

$$
\begin{gathered}
D_{+}|X(t)| \leq-\left(\lambda_{h} I_{v}+\nu_{h}-\alpha_{h} I_{h}+m_{h}-2 \alpha_{h} I_{h}\right)|X(t)|+\lambda_{h} S_{h} \frac{I_{v}}{I_{h}}\left(\frac{I_{h}}{I_{v}}(|Y(t)|+|Z(t)|)\right) \\
D_{+}|Y(t)| \leq \lambda_{v}\left(1-I_{v}\right)|X(t)|-\left(\lambda_{h} I_{v}+\nu_{h}-\alpha_{h} I_{h}+\lambda_{v} I_{h}+\mu_{v}\right)|Y(t)|+\alpha_{h} S_{h}|Z(t)| \\
D_{+}|Z(t)| \leq \lambda_{h} I_{v}|Y(t)|-\left(m_{h}-2 \alpha_{h} I_{h}+\lambda_{v} I_{h}+\mu_{v}\right)|Z(t)|
\end{gathered}
$$


Adicionando as desigualdades (2.53) e (2.54) e, usando o fato de, $\gamma_{h}$ e $1-S_{h}-I_{h}$ serem não negativos, obtemos:

$$
D_{+}[|Y(t)|+|Z(t)|] \leq \lambda_{v}\left(1-I_{v}\right)|X(t)|-\left(\nu_{h}-\alpha_{h} I_{h}+\lambda_{h} I_{+} \mu_{v}\right)(|Y(t)|+|Z(t)|)
$$

e, portanto,

$$
\begin{aligned}
D_{+}\left[\frac{I_{h}}{I_{v}}|Y(t)|+|Z(t)|\right] & =\left(\frac{I_{h}^{\prime}}{I_{h}}-\frac{I_{v}^{\prime}}{I_{v}}\right) \frac{I_{h}}{I_{v}}(|Y(t)|+|Z(t)|)+\frac{I_{h}}{I_{v}} D_{+}[|Y(t)|+|Z(t)|] \\
& \leq \frac{I_{h}}{I_{v}} \lambda_{v}\left(1-I_{v}\right)|X(t)|+\left(\frac{I_{h}^{\prime}}{I_{h}}-\frac{I_{v}^{\prime}}{I_{v}}-\nu_{h}+\alpha_{h} I_{h}-\lambda_{v} I_{h}-\mu_{v}\right) \frac{I_{h}}{I_{v}}(|Y(t)|+|Z(t)|) .
\end{aligned}
$$

Seja,

$$
\begin{gathered}
f_{1}(t)=\lambda_{h} S_{h} \frac{I_{v}}{I_{h}}-\left(\lambda_{h} I_{v}+\nu_{h}-\alpha_{h} I_{h}+m_{h}-2 \alpha_{h} I_{h}\right) \\
f_{2}(t)=\frac{I_{h}}{I_{v}} \lambda_{v}\left(1-I_{v}\right)+\frac{I_{h}^{\prime}}{I_{h}}-\frac{I_{v}^{\prime}}{I_{v}}-\nu_{h}+\alpha_{h} I_{h}-\lambda_{v} I_{h}-\mu_{v} .
\end{gathered}
$$

Da desigualdade (2.52) e da expressão (2.55), é possível mostrar conforme Li e Muldowney, em [LM95], que:

$$
D_{+} V(t) \leq \sup \left\{f_{1}(t), f_{2}(t)\right\} V(t) .
$$

Assim, após um rearranjo das equações do sistema (2.33), temos as seguintes relações

$$
\begin{gathered}
-\lambda_{h} I_{v}+\alpha_{h} I_{h}=\frac{S_{h}^{\prime}}{S_{h}}-\frac{\nu_{h}\left(1-S_{h}\right)}{S_{h}}, \\
-\frac{\lambda_{v} I_{h}}{I_{v}}\left(1-I_{v}\right)=\frac{I_{v}^{\prime}}{I_{v}}+\mu_{v}, \\
-\frac{\lambda_{v} S_{h} I_{v}}{I_{h}}=\frac{I_{h}^{\prime}}{I_{h}}+m_{h}-\alpha_{h} I_{h},
\end{gathered}
$$

as quais, aplicadas as expressões de $f_{1}(t)$ e $f_{2}(t)$, fornecem a seguinte desigualdade:

$$
\begin{aligned}
\sup \left\{f_{1}(t), f_{2}(t)\right\} & \leq \frac{I_{h}^{\prime}}{I_{h}}+\frac{S_{h}^{\prime}}{S_{h}}-\nu_{h}+\alpha_{h} I_{h} \\
& \leq \frac{I_{h}^{\prime}}{I_{h}}+\frac{S_{h}^{\prime}}{S_{h}}-\nu_{h}+\alpha_{h} .
\end{aligned}
$$

Substituindo (2.57) em (2.56), temos

$$
D_{+} V(t) \leq \frac{I_{h}^{\prime}}{I_{h}}+\frac{S_{h}^{\prime}}{S_{h}}-\nu_{h}+\alpha_{h}
$$

Como a equação que define o sistema (2.50) é analítica, as soluções deste sistema também serão analíticas. Portanto, existem intervalos $\left[t_{i}, t_{i+1}\right]$, os quais, $V(t)$ é diferenciável. Assim, integrando-se a desigualdade abaixo

$$
\frac{V^{\prime}(t)}{V(t)} \leq \frac{I_{h}^{\prime}}{I_{h}}+\frac{S_{h}^{\prime}}{S_{h}}-\nu_{h}+\alpha_{h}
$$

obtemos: 


$$
\ln \left(\frac{V(t)}{V(0)}\right) \leq-\left(\nu_{h}-\alpha_{h}\right) t+\ln \left(\frac{I_{h}(t)}{I_{h}(0)}\right)+\ln \left(\frac{S_{h}(t)}{S_{h}(0)} .\right)
$$

Desta desigualdade, implica

$$
\frac{V(t)}{V(0)} \leq e^{-\left(\nu_{h}-\alpha_{h}\right) t} \cdot\left(\frac{I_{h}(t)}{I_{h}(0)}+\frac{S_{h}(t)}{S_{h}(0)}\right),
$$

e portanto,

$$
V(t) \leq V(0)\left(I_{h}(t)+S_{h}(t)\right) e^{-\left(\nu_{h}-\alpha_{h}\right) t} \leq V(0) e^{-\left(\nu_{h}-\alpha_{h}\right) t} .
$$

Como $\nu_{h}-\alpha_{h}>0$, segue que $V(t) \rightarrow 0$ quando $t \rightarrow \infty$ e portanto, o sistema (2.50) é assintoticamente estável. Consequentemente, através deste resultado mostramos que o sistema (2.33) possui a propriedade da estabilidade de órbitas e periódicas.

Portanto, concluímos que o equilíbrio endêmico, $E_{2}$ é globalmente estável para $R_{o}>1$.

Do mesmo modo, além da estabilidade global de $E_{2}$ podemos mostrar que se $R_{o}>1$ existe uma região assintoticamente estável para o equilíbrio endêmico.

Proposição 2.2.7. Se $R_{o}>1$ então $\Omega-\left\{\left(S_{h}, 0,0\right): 0 \leq S_{h} \leq 1\right\}$ é uma região assintoticamente estável para $E_{2}$. Além disso, todas as soluções começando no eixo $S_{h}$ se aproximam do equilíbrio livre de doença $E_{1}$.

Prova: Pela transversalidade do campo em $\Omega-\left\{\left(S_{h}, 0,0\right): 0 \leq S_{h} \leq 1\right\}$ e pelo teorema 2.2.6 segue a primeira parte da proposição. A segunda parte segue diretamente da proposição 2.2.2.

\subsection{Modelo 3}

\subsubsection{Descrição do modelo matemático}

O análise matemática do modelo 3, o qual será apresentado nesta subseção, segue a metodologia proposta nos trabalhos de, Garba, et al., em [GGB08], Li e Zhen, em [LJ14] e, Castillo-Chavez e Song, em [CCS04]. O modelo 3 foi construído sob a hipótese de que a população humana é expressa por uma função de crescimento logístico. O diagrama de fluxo que descreve a dinâmica do modelo 3, é representado pela Figura 2.3:

Sejam $N_{h}$ e $N_{v}$, as populações totais de humanos e vetores, respectivamente. Do mesmo modo, $\bar{S}_{h}, \bar{I}_{h}, \bar{R}_{h}$, $\bar{S}_{v}$ e $\bar{I}_{v}$ representam as subpopulações de humanos suscetíveis, humanos infectados, humanos recuperados, vetores suscetíveis e vetores infectados, respectivamente.

O recrutamento de humanos suscetíveis, é gerado à uma taxa de

$$
\left(\lambda-\frac{\gamma N_{h}}{K}\right) N_{h}
$$

tais que, $\lambda$ é a taxa de nascimentos (considerada constante), $\gamma$ é a taxa de crescimento da população, dada pela diferença entre nascimentos e mortes, $\left(\lambda-\mu_{h}\right)$, e $K$ corresponde a capacidade de suporte, ou seja, o número máximo de indivíduos de uma determinada população que o ambiente consegue sustentar.

Um humano suscetível adquire infecção à taxa:

$$
\frac{b \beta_{h} \bar{S}_{h} \bar{I}_{v}}{N_{h}}
$$

cujo, $\beta_{h}$ corresponde a probabilidade de transmissão de um vetor infectado para um humano suscetível e o parâmetro $b$ corresponde ao número médio de picadas dadas por um único vetor, por humano, por dia. 


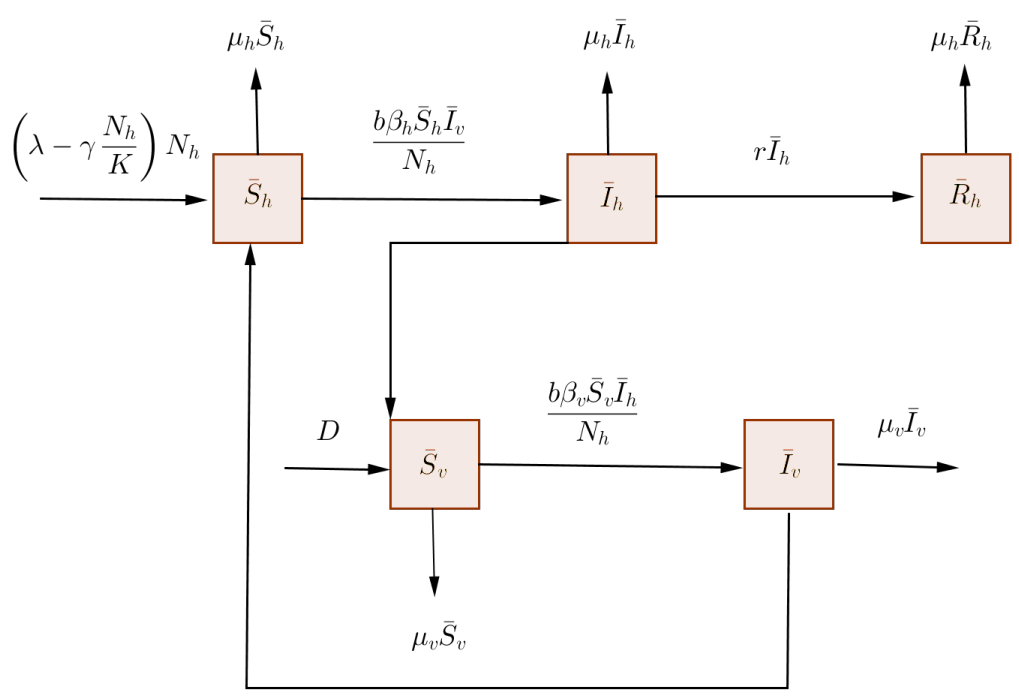

Figura 2.3: Dinâmica de propagação do dengue referente ao modelo 3

O compartimento $\bar{R}_{h}$ inclui, tanto os humanos que se recuperam da doença à taxa $r$ quanto, os que morrem em função dela (como a taxa de morte associada ao vírus do dengue é baixa, vamos omitíla).

Com relação a população de vetores, vetores suscetíveis são recrutados à uma taxa constante $D$ que, independe do tamanho atual da população de vetores. Além disso, vetores suscetíveis adquirem infecção à taxa:

$$
\frac{b \beta_{v} \bar{S}_{v} \bar{I}_{h}}{N_{h}}
$$

onde, $\beta_{v}$ é a probabilidade de transmissão de um humano infectado para um vetor suscetível.

Os vetores nunca recuperam-se de uma infecção e a sua taxa de morte é representada pelo parâmetro $\mu_{v}$.

Para o modelo 3, assumem-se como hipóteses:

- Só existe um sorotipo de vírus circulando;

- As populações de humanos e de vetores são distribuídas homogeneamente;

- Não existe transmissão vertical para humanos;

- Todo vetor pode picar um humano com igual probabilidade;

- Humanos que recuperam-se, adquirem imunidade permanente contra a doença.

Portanto, baseado nestas hipóteses, as equações diferenciais que descrevem o modelo em estudo são dadas pelo sistema: 


$$
\left\{\begin{array}{l}
\frac{d \bar{S}_{h}}{d t}=\left(\lambda-\frac{\gamma N_{h}}{K}\right) N_{h}-\frac{b \beta_{h} \bar{S}_{h} \bar{I}_{v}}{N_{h}}-\mu_{h} \bar{S}_{h} \\
\frac{d \bar{I}_{h}}{d t}=\frac{b \beta_{h} \bar{S}_{h} \bar{I}_{v}}{N_{h}}-\left(\mu_{h}+r\right) \bar{I}_{h} \\
\frac{d \bar{R}_{h}}{d t}=r \bar{I}_{h}-\mu_{h} \bar{R}_{h} \\
\frac{d \bar{S}_{v}}{d t}=D-\frac{b \beta_{v} \bar{S}_{v} \bar{I}_{h}}{N_{h}}-\mu_{v} \bar{S}_{v} \\
\frac{d \bar{I}_{v}}{d t}=\frac{b \beta_{v} \bar{S}_{v} \bar{I}_{h}}{N_{h}}-\mu_{v} \bar{I}_{v}
\end{array}\right.
$$

cujas, condições iniciais são: $\bar{S}_{h}(0)=S_{h o} \geq 0, \bar{I}_{h}(0)=I_{h o} \geq 0, \bar{R}_{h}(0)=R_{h o} \geq 0, \bar{S}_{v}(0)=S_{v o} \geq 0$, $I_{v}(0)=I_{v o} \geq 0$.

Além disso, como $N_{h}=\bar{S}_{h}+\bar{I}_{h}+\bar{R}_{h}$ e $N_{v}=\bar{S}_{v}+\bar{I}_{v}$, temos ainda duas equações para o sistema (2.59)

$$
\left\{\begin{array}{l}
\frac{d N_{h}}{d t}=\gamma\left(1-\frac{N_{h}}{K}\right) N_{h} \\
\frac{d N_{v}}{d t}=D-\mu_{v} N_{v}
\end{array}\right.
$$

cujas, condições iniciais são: $0 \leq N_{h}(0)=N_{h o} \leq K$ e $N_{v}(0)=N_{v o} \geq 0$.

Levando em consideração que a dinâmica da população de vetores é mais rápida que a de humanos, vamos considerar $N_{v}=\frac{D}{\mu_{v}}$. Sem perda de generalidade, podemos trabalhar com as proporções da população,

$$
S_{h}=\frac{\bar{S}_{h}}{N_{h}}, \quad I_{h}=\frac{\bar{I}_{h}}{N_{h}}, \quad R_{h}=\frac{\bar{R}_{h}}{N_{h}}, \quad S_{v}=\frac{\bar{S}_{v}}{D / \mu_{v}} \quad \text { e } \quad I_{v}=\frac{\bar{I}_{v}}{D / \mu_{v}} .
$$

Além disso, como estamos assumindo que a população de vetores é constante, a equação que descreve a dinâmica dos vetores suscetíveis torna-se redudante, podemos reescrever o sistema (2.59) como sendo:

$$
(G)\left\{\begin{array}{l}
S_{h}^{\prime}(t)=\left(\lambda-\frac{\gamma N_{h}}{K}\right)-b \beta_{h} S_{h} I_{v} \frac{D / \mu_{v}}{N_{h}}-\mu_{h} S_{h}-\gamma S_{h}\left(1-\frac{N_{h}}{K}\right) \\
I_{h}^{\prime}(t)=b \beta_{h} S_{h} I_{v} \frac{D / \mu_{v}}{N_{h}}-\left(\mu_{h}+r\right) I_{h}-\gamma I_{h}\left(1-\frac{N_{h}}{K}\right) \\
R_{h}^{\prime}(t)=r I_{h}-\mu_{h} R_{h}-\gamma R_{h}\left(1-\frac{N_{h}}{K}\right) \\
I_{v}^{\prime}(t)=b \beta_{v}\left(1-I_{v}\right) I_{h}-\mu_{v} I_{v}
\end{array}\right.
$$

cujas, condições iniciais são: $S_{h}(0) \geq 0, I_{h}(0) \geq 0, R_{h}(0) \geq 0, I_{v}(0) \geq 0$ e $0 \leq N_{h}(0) \leq K$.

A região de interesse biológico

$$
\Omega=\left\{\left(S_{h}, I_{h}, R_{h}, I_{v}\right) \in \mathbb{R}^{4} / 0 \leq S_{h}, 0 \leq I_{h}, 0 \leq R_{h}, S_{h}+I_{h}+R_{h}=1,0 \leq I_{v} \leq 1\right\},
$$

é positivamente invariante. De fato, mostraremos que a soluções do sistema (2.61), são não negativas para todo $t>0$ e limitadas em $\Omega$.

Observe que, $S_{h}(t)>0$ para todo $t \geq 0$. Do contrário, se isso não contece, existiria um $t^{*}>0$ tal que, $S_{h}\left(t^{*}\right)=0, S_{h}^{\prime}\left(t^{*}\right) \leq 0$ e $S_{h}, I_{h}, R_{h}, I_{v}>0$, para $0<t<t^{*}$. Por outro lado, do sistema (2.61) temos: 


$$
\begin{aligned}
S_{h}^{\prime}\left(t^{*}\right) & =\left(\lambda-\gamma \frac{N_{h}\left(t^{*}\right)}{K}\right)-b \beta_{h} S_{h}\left(t^{*}\right) I_{v}\left(t^{*}\right) \frac{D / \mu_{v}}{N_{h}\left(t^{*}\right)}-\mu_{h} S_{h}\left(t^{*}\right)-\gamma S_{h}\left(t^{*}\right)\left(1-\frac{N_{h}\left(t^{*}\right)}{K}\right) \\
& =\left(\lambda-\gamma \frac{N_{h}\left(t^{*}\right)}{K}\right) \\
& =\lambda-\lambda \frac{N_{h}\left(t^{*}\right)}{K}+\mu_{h} \frac{N_{h}\left(t^{*}\right)}{K} \\
& =\lambda\left(1-\frac{N_{h}\left(t^{*}\right)}{K}\right)+\mu_{h} \frac{N_{h}\left(t^{*}\right)}{K}>0
\end{aligned}
$$

pois, por hipótese, $N_{h} \leq K$. Portanto, $S_{h}(t)>0$, para todo $t \geq 0$.

Assumindo que, existe $t^{*}=\sup \left\{t>0: S_{h}, I_{h}, R_{h}, I_{v}>0\right\}$, integrando-se a segunda equação do sistema (2.61), de 0 à $t^{*}$, vamos obter:

$$
I_{h}\left(t^{*}\right)=I_{h}(0) e^{-\left(\lambda+r-\gamma \frac{N_{h}}{K}\right) t^{*}}+e^{-\left(\lambda+r-\gamma \frac{N_{h}}{K}\right) t^{*}} \int_{0}^{t^{*}} b \beta_{h} S_{h}(\theta) I_{v}(\theta) \frac{D / \mu_{v}}{N_{h}(\theta)} e^{\left(\lambda+r-\gamma \frac{N_{h}(\theta)}{K}\right) \theta} d \theta>0
$$

e portanto, $I_{h}(t)>0$, para todo $t \geq 0$.

Repetindo o mesmo argumento para $R_{h}$, vamos obter que $R_{h}(t)>0$, para todo $t \geq 0$. Finalmente da equação de $I_{v}^{\prime}(t)$, é possível obter:

$$
\begin{aligned}
I_{v}^{\prime}(t) & =b \beta_{v}\left(1-I_{v}\right) I_{h}-\mu_{v} I_{v} \\
& \geq-\mu_{v} I_{v}(t)
\end{aligned}
$$

e, portanto,

$$
I_{v}(t) \geq I_{v}(0) e^{-\mu_{v} t} \geq 0 .
$$

Com isso concluímos que todas as soluções do sistema (2.61), são não negativas para todo $t>0$.

Nosso próximo passo para concluírmos que $\Omega$ é positivamente invariante, é garantirmos que as soluções do sistema (2.61), são limitadas em $\Omega$. Para isso, seja $\left\{S_{h}, I_{h}, R_{h}, I_{v}\right\} \in \mathbb{R}_{+}^{4}$, soluções do sistema (2.61), com condições iniciais não negativas.

Pelo teorema da desigualdade diferencial

$$
\lim _{t \rightarrow \infty} S_{h}(t) \leq 1 \quad \text { e } \quad \lim _{t \rightarrow \infty} I_{v}(t) \leq 1 .
$$

Além disso, sabemos que $N_{h}=S_{h}+I_{h}+R_{h}=1$ e $N_{v}=S_{v}+I_{v}=1$. Logo, como as soluções $S_{h}, I_{h}, R_{h}, I_{v}$ são não negativas para todo $t>0$, pela desigualdade (2.62), concluímos que as soluções do sistema (2.61), são limitadas em $\Omega$, e portanto $\Omega$ é positivamente invariante.

A prova deste argumento, nos garante que o sistema (2.3) é bem posto, tanto matematicamente quanto, epidemiologicamente.

\subsubsection{Pontos de equilíbrio e Número de Reprodutibilidade Basal}

Nesta subseção, iremos obter as expressões para os pontos de equilíbrio associados ao sistema (2.61). Conforme descrição anterior usaremos o parâmetro $R_{o}$ como sendo:

$$
R_{o}=\frac{n b^{2} \beta_{h} \beta_{v}}{\mu_{v}(\lambda+r-\gamma \alpha)} .
$$

Por questão de simplicidade vamos considerar $\alpha=\frac{N_{h}}{K}$ e $n=\frac{N_{v}}{N_{h}}=\frac{D / \mu_{v}}{N_{h}}$. Assim, o sistema (2.61), no equilíbrio, pode ser reescrito como: 


$$
\begin{aligned}
& 0=(\lambda-\gamma \alpha)\left(1-S_{h}\right)-n b \beta_{h} S_{h} I_{v} \\
& 0=n b \beta_{h} S_{h} I_{v}-(\lambda+r-\gamma \alpha) I_{h} \\
& 0=r I_{h}-(\lambda-\gamma \alpha) R_{h} \\
& 0=b \beta_{v}\left(1-I_{v}\right) I_{h}-\mu_{v} I_{v}
\end{aligned}
$$

De imediato, se $I_{h}=I_{v}=0$ obtemos o equilíbrio livre de doença, $E_{1}=(1,0,0,0)$.

Por outro lado, considerando $I_{h} \neq 0$, podemos obter após algumas manipulações algébricas as expressões para o equilíbrio endêmico $E_{2}=\left(S_{h}^{*}, I_{h}^{*}, R_{h}^{*}, I_{v}^{*}\right)$, como:

$$
\begin{gathered}
S_{h}^{*}=\frac{b \beta_{v} M+1}{R_{o}+b \beta_{v} M} \\
I_{h}^{*}=\mu_{v} M\left[\frac{R_{o}-1}{R_{o}+b \beta_{v} M}\right] \\
R_{h}^{*}=\frac{r \mu_{v} M}{(\lambda-\gamma \alpha)}\left[\frac{R_{o}-1}{R_{o}+b \beta_{v} M}\right] \\
I_{v}^{*}=(\lambda-\gamma \alpha)\left[\frac{R_{o}-1}{R_{o}(\lambda-\gamma \alpha)+n b \beta_{h}}\right]
\end{gathered}
$$

tais que, $R_{o}=\frac{n b^{2} \beta_{h} \beta_{v}}{\mu_{v}(\lambda+r-\gamma \alpha)}$ e $M=\frac{\lambda-\gamma \alpha}{\mu_{v}(\lambda+r-\gamma \alpha)}$.

De fato, escrevendo $S_{h}^{*}, R_{h}^{*}, I_{v}^{*}$ em função de $I_{h}^{*}$, vamos obter:

$$
\begin{gathered}
S_{h}^{*}=\frac{(\lambda-\gamma \alpha)-(\lambda+r-\gamma \alpha) I_{h}^{*}}{(\lambda-\gamma \alpha)} \\
R_{h}^{*}=\frac{r I_{h}^{*}}{(\lambda-\gamma \alpha)} \\
I_{v}^{*}=\frac{b \beta_{v} I_{h}^{*}}{b \beta_{v} I_{h}^{*}+\mu_{v}} .
\end{gathered}
$$

Substituindo as expressões de $S_{h}^{*}$ e $I_{v}^{*}$, na segunda equação do sistema (2.64), vamos ter que $I_{h}^{*}$, deve ser solução da equação quadrática:

$$
A I_{h}^{* 2}+B I_{h}^{*}+C=0
$$

cujos,

$$
\begin{aligned}
& A=(\lambda+r-\gamma \alpha) b \beta_{v}\left[(\lambda-\gamma \alpha)+n b \beta_{h}\right] \\
& B=(\lambda-\gamma \alpha) \mu_{v}(\lambda+r-\gamma \alpha)\left[1-R_{o}\right] \\
& C=0 .
\end{aligned}
$$

Da equação (2.69), segue que $\left(A I_{h}^{*}+B\right) I_{h}^{*}=0$. Portanto, as soluções de (2.69) são tais que, $I_{h}^{*}=0$, o qual corresponde ao equilíbrio livre de doença, e $f\left(I_{h}^{*}\right)=0$ que corresponde ao único equilíbrio endêmico quando, $R_{o}>1, A>0$ e $B<0$. Então:

$$
I_{h}^{*}=\frac{-B}{A}=\frac{\mu_{v}(\lambda-\gamma \alpha)\left[R_{o}-1\right]}{\mu_{v}(\lambda+r-\gamma \alpha) R_{o}+b \beta_{v}(\lambda-\gamma \alpha)}=\mu_{v} M\left[\frac{R_{o}-1}{R_{o}+b \beta_{v} M}\right] .
$$




\subsubsection{Análise de estabilidade dos pontos de equilíbrio}

Nesta subseção faremos a análise de estabilidade local do equilíbrio livre de doença através da verificação do critério de Routh-Hurwitz e para a estabilidade global usaremos uma função de Lyapunov. Para a estabilidade local de $E_{2}$ usaremos argumentos da Teoria de Bifurcação. Para a estabilidade global repetiremos os mesmos argumentos descritos na Subseção 2.1.3.

Proposição 2.3.1. O equilıbrio livre de doença $E_{1}=(1,0,0,0)$ é localmente assintoticamente estável para $R_{o}<1$ e instável para $R_{o}>1$.

Prova: Seja $D G\left(E_{1}\right)$, a matriz jacobiana do sistema (2.61) aplicada ao equilílibrio livre de doença

$$
D G\left(E_{1}\right)=\left[\begin{array}{cccc}
-(\lambda-\gamma \alpha) & 0 & 0 & -n b \beta_{h} \\
0 & -(\lambda+r-\gamma \alpha) & 0 & n b \beta_{h} \\
0 & r & -(\lambda-\gamma \alpha) & 0 \\
0 & b \beta_{v} & 0 & -\mu_{v}
\end{array}\right]
$$

onde, $\alpha=\frac{N_{h}}{K}$ e $n=\frac{N_{v}}{N_{h}}=\frac{D / \mu_{v}}{N_{h}}$.

Seja $P(\Lambda)$, o polinômio característico associado a matriz (2.70)

$$
P(\Lambda)=P_{1}(\Lambda) P_{2}(\Lambda)
$$

onde,

$$
\begin{aligned}
& P_{1}(\Lambda)=(\lambda-\gamma \alpha+\Lambda)^{2} \\
& P_{2}(\Lambda)=(\lambda+r-\gamma \alpha+\Lambda)\left(\mu_{v}+\Lambda\right)-n b^{2} \beta_{h} \beta_{v} .
\end{aligned}
$$

De $P_{1}(\Lambda)$, obtemos como autovalores:

$$
\Lambda_{1}=\Lambda_{2}=-(\lambda-\gamma \alpha)<0 .
$$

De fato, $(\lambda-\gamma \alpha)>0$ pois, $N_{h}<K$ e $\mu_{h}$ é não negativo. Finalmente, de $P_{2}(\Lambda)$, temos:

$$
\begin{aligned}
P_{2}(\Lambda) & =(\lambda+r-\gamma \alpha+\Lambda)\left(\mu_{v}+\Lambda\right)-n b^{2} \beta_{h} \beta_{v} \\
& =\Lambda^{2}+\left(\lambda+r-\gamma \alpha+\mu_{v}\right) \Lambda+(\lambda+r-\gamma \alpha) \mu_{v}-n b^{2} \beta_{h} \beta_{v} \\
& =\Lambda^{2}+\left(\lambda+r-\gamma \alpha+\mu_{v}\right) \Lambda+(\lambda+r-\gamma \alpha) \mu_{v}\left(1-R_{o}\right) .
\end{aligned}
$$

Portanto, pelo critério de Routh-Hurwitz, $P_{2}(\Lambda)$ tem todas as suas raízes com parte real negativa se, $R_{o}<1$. Isso implica que, $E_{1}$ é localmente assintoticamente estável para $R_{o}<1$ e instável para $R_{o}>1$.

Proposição 2.3.2. Se $R_{o} \leq 1$, então o equilíbrio livre de doença $E_{1}=(1,0,0,0)$ é globalmente assintoticamente estável em $\Omega$.

Prova: Seja, 


$$
\left(G_{1}\right)\left\{\begin{array}{l}
S_{h}^{\prime}(t)=(\lambda-\gamma \alpha)\left(1-S_{h}\right)-n b \beta_{h} S_{h} I_{v} \\
I_{h}^{\prime}(t)=n b \beta_{h} S_{h} I_{v}-(\lambda+r-\gamma \alpha) I_{h} \\
R_{h}^{\prime}(t)=r I_{h}-(\lambda-\gamma \alpha) R_{h} \\
I_{v}^{\prime}(t)=b \beta_{v}\left(1-I_{v}\right) I_{h}-\mu_{v} I_{v}
\end{array}\right.
$$

o sistema de equações diferenciais ordinárias referente ao modelo 3.

Pelo fato da equação diferencial de $R_{h}$ ser desacoplada do sistema, somos capazes de expressar analiticamente a sua solução, da forma

$$
R_{h}(t)=R_{h}(0) e^{-(\lambda-\gamma \alpha) t}+r e^{-(\lambda-\gamma \alpha) t} \int_{0}^{t} I_{h}(s) e^{-(\lambda-\gamma \alpha) s} d s .
$$

Da solução de $R_{h}^{\prime}$, podemos concluir que, a estabilidade na variável $R_{h}$ estará bem definida dependendo do comportamento assintótico da solução de $I_{h}$. Assim, se garantirmos que a variável $I_{h}$ é assintoticamente estável, podemos concluir que a variável $R_{h}$ também é assintoticamente estável.

Desta forma, considere a função de Lyapunov, $V:\left\{\left(S_{h}, I_{h}, I_{v}\right) \in \Omega: S_{h}>0\right\} \rightarrow \mathbb{R}$, dada por:

$$
V\left(S_{h}, I_{h}, I_{v}\right)=\left(S_{h}-1-\log \left(S_{h}\right)\right)+I_{h}+\frac{(\lambda+r-\gamma \alpha)}{b \beta_{v}} I_{v} \geq 0 .
$$

Observe que, $V$ é $C^{1}$ no interior de $\Omega$ e $E_{1}$ é um mínimo global de $V$ em $\Omega$. Além disso, $V(1,0,0)=0$.

Calculando a derivada de $V$ em função de $t$, vamos obter:

$$
V^{\prime}\left(S_{h}, I_{h}, I_{v}\right)=\left(1-\frac{1}{S_{h}}\right) S_{h}^{\prime}+I_{h}^{\prime}+\frac{(\lambda+r-\gamma \alpha)}{b \beta_{v}} I_{v}^{\prime} .
$$

Mas,

$$
\begin{aligned}
S_{h}^{\prime} & =(\lambda-\gamma \alpha)\left(1-S_{h}\right)-n b \beta_{h} S_{h} I_{v} \\
I_{h}^{\prime} & =n b \beta_{h} S_{h} I_{v}-(\lambda+r-\gamma \alpha) I_{h} \\
I_{v}^{\prime} & =b \beta_{v}\left(1-I_{v}\right) I_{h}-\mu_{v} I_{v} .
\end{aligned}
$$

Assim, substituindo as expressões, $S_{h}^{\prime}, I_{h}^{\prime}, I_{v}^{\prime}$, pelas equações acima, obtém-se:

$$
\begin{aligned}
V^{\prime}\left(S_{h}, I_{h}, I_{v}\right) & =\left(1-\frac{1}{S_{h}}\right)\left[(\lambda-\gamma \alpha)\left(1-S_{h}\right)-n b \beta_{h} S_{h} I_{v}\right]+\left[n b \beta_{h} S_{h} I_{v}-(\lambda+r-\gamma \alpha) I_{h}\right] \\
& +\frac{(\lambda+r-\gamma \alpha)}{b \beta_{v}}\left[b \beta_{v}\left(1-I_{v}\right) I_{h}-\mu_{v} I_{v}\right] \\
& =-(\lambda-\gamma \alpha) \frac{\left(S_{h}-1\right)^{2}}{S_{h}}+n b \beta_{h} I_{v}-(\lambda+r-\gamma \alpha) I_{v} I_{h}-\frac{\mu_{v}(\lambda+r-\gamma \alpha)}{b \beta_{v}} I_{v} .
\end{aligned}
$$

Note que, $(\lambda-\gamma \alpha)>0$ pois, $N_{h} \leq K$. De fato,

$$
(\lambda-\gamma \alpha)=\left(\lambda-\left(\lambda-\mu_{h}\right) \frac{N_{h}}{K}\right)=\lambda\left(1-\frac{N_{h}}{K}\right)+\mu_{h} \frac{N_{h}}{K} .
$$


Assim, após algumas manipulações algébricas, tem-se:

$$
V^{\prime}=-(\lambda-\gamma \alpha) \frac{\left(S_{h}-1\right)^{2}}{S_{h}}-(\lambda+r-\gamma \alpha) I_{v} I_{h}-\frac{\mu_{v}(\lambda+r-\gamma \alpha)}{b \beta_{v}}\left(1-R_{o}\right) I_{v} \leq 0
$$

Portanto, se $R_{o} \leq 1$ então, $V^{\prime}\left(S_{h}, I_{h}, R_{h}, I_{v}\right)<0$ para todo $S_{h}, I_{h}, R_{h}, I_{v}>0$. Note ainda que, o subconjunto de $\Omega$ onde $V^{\prime}=0$ é definido pelas condições: $S_{h}=1, I_{h}=0$ e $R_{o}=1$ ou, $S_{h}=1, I_{v}=0$ e $R_{o}<1$. Logo, o maior conjunto compacto invariante $\left\{\left(S_{h}, I_{h}, I_{v}\right) \in \Omega: V^{\prime}=0\right\}$ é $\left\{E_{1}\right\}$ onde, $E_{1}$ é o equilíbrio livre de doença.

Finalmente, pelo Princípio da Invariância de Lasalle 2.1.2, $E_{1}$ é globalmente estável em $\Omega$ e isto conclui a prova da proposição.

Para avaliar a estabilidade local do equilíbrio endêmico utilizaremos argumentos da teoria de bifurcações. Através da escolha do parâmetro de bifurcação, vamos obter que o polinômio característico associado a linearização do sistema, em torno do equilíbrio, terá como autovalor um ponto não hiperbólico. Nesse aspecto, argumentos da teoria da Variedade Central serão muito úteis para verificarmos a estabilidade de $E_{2}$, através do estudo do diagrama de bifurcação obtido em $R_{o}=1$.

Para grande parte dos modelos epidemiológicos, existem duas distintas bifurcações em $R_{o}=1$, forward e backward. A bifurcação para frente (forward), aparece quando $R_{o}$ excede o valor 1 para cima. Dessa bifurcação decorre o aparecimento de um pequeno equilíbrio positivo assintoticamente estável (equilíbrio endêmico), que irá fazer com que o equilíbrio livre de doença perca a sua estabilidade. Neste caso, a doença só conseguirá invadir a população se $R_{o}$ for estritamente maior do que 1 ([CCS04], [DHCC98]).

Por outro lado, a bifurcação para trás (backward) ocorre quando $R_{o}<1$. Neste caso, temos o aparecimento de um pequeno equilíbrio positivo instável, enquanto que, o equilíbrio livre de doença e um grande equilíbrio positivo são assintoticamente estáveis ([CCS04]). Epidemiologicamente, ter uma bifurcação para trás implica que, reduzir o valor de $R_{o}$ para ser menor do que uma unidade não será suficiente para eliminar a doença da população ([DHCC98]).

Recentes estudos com modelos matemáticos que apresentam bifurcação para trás foram trabalhados por Hadeler e Castillo-Chavez, (1992); Dushoff, (1998), em modelos de doenças fatais e mais recentemente van den Driessche e Watmough, têm mostrado a existência deste comportamento em modelos com delay ([CCS04]). Brauer, em [Bra04], aborda a existência deste tipo de bifurcação em modelos que incluem vacinação.

Neste sentido, argumentos da teoria da Variedade Central têm sido usados para decidir sobre a estabilidade do equilíbrio não hiperbólico (isto ocorre uma vez que, a linearização em torno do equilíbrio tem, pelo menos, um autovalor com parte real zero). Esta teoria pode não somente determinar a estabilidade local, como também investigar a existência de outro equilíbrio, derivado da bifurcação deste equilíbrio não hiperbólico ([CCS04], [Car12]).

Para descrevê-la, vamos considerar a metodologia conforme descrita em Castillo-Chavez, et al., em [CCS04]. Considere o sistema geral de EDO's, com um parâmetro $\phi$

$$
\frac{d x}{d t}=f(x, \phi) \quad f: \mathbb{R}^{n} \times \mathbb{R} \rightarrow \mathbb{R}^{n} \quad \text { e } \quad f \in C^{2}\left(\mathbb{R}^{n} \times \mathbb{R}^{n}\right)
$$

Sem perda de generalidade, assumimos que o zero é um equilíbrio do sistema (2.74) para quaisquer valores de $\phi$, ou seja, $f(0, \phi) \equiv 0$ para toda $\phi$.

Teorema 2.3.3. Assumindo que:

i) $A=D_{x} f(0,0)=\left(\frac{\partial f_{i}(0,0)}{\partial_{x i}}\right), i=1, \cdots, n$ é a linearização do sistema (2.74) em torno do equilíbrio zero com $\phi$ avaliada no zero. Zero é um autovalor simples de A e os demais autovalores têm parte real negativa; 
ii) A matriz A tem um autovetor à direita $v$ e um autovetor à esquerda u, correspondentes ao autovalor zero.

Seja $f_{k}$ a k-ésima componente de $f$ e

$$
\begin{aligned}
& a=\sum_{k, i, j=1}^{n} u_{k} v_{i} v_{j} \frac{\partial^{2} f_{k(0,0)}}{\partial_{x i} \partial_{x j}} e \\
& b=\sum_{k, i=1}^{n} u_{k} v_{i} \frac{\partial^{2} f_{k(0,0)}}{\partial_{x i} \partial_{\phi}}
\end{aligned}
$$

então a dinâmica local de (2.74) em torno do zero é totalmente governada pelo sinal de a e b. Assim, podemos ter os seguintes casos:

Caso 1) $a>0, b>0$. Quando $\phi<0$ com $|\phi|<<1$, implica que o zero é assintoticamente estável e existe um equilíbrio instável positivo; quando $0<|\phi|<<1$, o zero é instável e existe um equilíbrio negativo localmente assintoticamente estável;

Caso 2) $a<0, b<0$. Quando $\phi<0$ com $|\phi|<<1$, implica que o zero é instável; quando $0<|\phi|<<1$, o zero é assintoticamente estável e existe um equilíbrio positivo instável;

Caso 3) $a>0, b<0$. Quando $\phi<0$ com $|\phi|<<1$, o zero é instável e existe um equilibrio negativo que é localmente assintoticamente estável; quando $0<|\phi|<<1$, o zero é estável e um equilibrio positivo instável surge;

Caso 4) $a<0, b>0$. Quando $\phi$ muda de negativo para positivo o zero muda a estabilidade de estável para instável, devido ao equilíbrio negativo que começa a ser positivo e localmente assintoticamente estável.

Em particular se, $a>0$ e $b>0$ a bifurcação para trás ocorre em $\phi=0$.

Prova: A prova deste teorema pode ser obtida em [CCS04].

Na Figura 2.4 temos um resumo dos 4 casos descritos no teorema 2.3.3. No diagrama de bifurcação, os pontos de equilíbrio $x^{*}$, são representados no eixo vertical e, no eixo horizontal temos a representação do parâmetro $\phi$. As linhas sólidas representam a estabilidade, $S$ e, as linhas tracejadas representam a instabilidade, $U$, ([CCS04]).

À priori, para aplicarmos o Teorema 2.3.3 ao modelo em estudo, precisamos fazer uma mudança de variáveis. Seja, $S_{h}=x_{1}, I_{h}=x_{2}, R_{h}=x_{3}$ e $I_{v}=x_{4}$. Usaremos a notação vetorial $X=\left(x_{1}, x_{2}, x_{3}, x_{4}\right)^{T}$, sendo assim, é possível reescrever o sistema (2.72), da seguinte forma:

$$
G_{1}\left(x_{1}, x_{2}, x_{3}, x_{4}\right)=\left\{\begin{array}{l}
x_{1}^{\prime}(t)=f_{1}=(\lambda-\gamma \alpha)\left(1-x_{1}\right)-n b \beta_{h} x_{1} x_{4} \\
x_{2}^{\prime}(t)=f_{2}=n b \beta_{h} x_{1} x_{4}-(\lambda+r-\gamma \alpha) x_{2} \\
x_{3}^{\prime}(t)=f_{3}=r x_{2}-(\lambda-\gamma \alpha) x_{3} \\
x_{4}^{\prime}(t)=f_{4}=b \beta_{v}\left(1-x_{4}\right) x_{2}-\mu_{v} x_{4} .
\end{array}\right.
$$

Para inferirmos sobre a estabilidade local do equilíbrio endêmico, precisaremos olhar inicialmente para o equilíbrio livre de doença (de acordo com as hipóteses do teorema 2.3.3, a equação 2.74, tem como ponto de equilíbrio o equilíbrio trivial). Ao calcularmos a matriz jacobiana referente ao sistema (2.77), aplicada ao equilíbrio livre de doença, obteremos a mesma matriz como em (2.70).

O parâmetro de bifurcação é escolhido dentro do espaço dos parâmetros como, o parâmetro que, ocasiona mudanças significativas na bifurcação. Para o sistema (2.77), $\beta_{h}$ é o parâmetro de bifurcação escolhido. Então ao tomarmos a expressão $R_{o}=1$, vamos obter: 


\begin{tabular}{|c|c|c|c|}
\hline $\mathbf{a} \mathbf{e} \mathbf{b}$ & estabilidade no 0 & estabilidade e sinal de $x^{*}$ & diagrama \\
\hline$a>0, b>0$ & $\begin{array}{ll}\phi<0, & \mathrm{~S} \\
\phi>0, & \mathrm{U}\end{array}$ & $\begin{array}{ll}\phi<0, x^{*}>0, & \mathrm{U} \\
\phi>0, x^{*}<0, & \mathrm{~S}\end{array}$ & \\
\hline$a<0, b<0$ & $\begin{array}{ll}\phi<0, & \mathrm{U} \\
\phi>0, & \mathrm{~S}\end{array}$ & $\begin{array}{ll}\phi<0, x^{*}>0, & \mathrm{~S} \\
\phi>0, x^{*}<0, & \mathrm{U}\end{array}$ & \\
\hline$a<0, b>0$ & $\begin{array}{ll}\phi<0, & \mathrm{~S} \\
\phi>0, & \mathrm{U}\end{array}$ & $\begin{array}{ll}\phi<0, x^{*}<0, & \mathrm{U} \\
\phi>0, x^{*}>0, & \mathrm{~S}\end{array}$ & \\
\hline$a>0, b<0$ & $\begin{array}{ll}\phi<0, & \mathrm{U} \\
\phi>0, & \mathrm{~S}\end{array}$ & $\begin{array}{ll}\phi<0, x^{*}<0, & \mathrm{~S} \\
\phi>0, x^{*}>0, & \mathrm{U}\end{array}$ & \\
\hline
\end{tabular}

Figura 2.4: Resumo dos casos descritos no Teorema 2.3.3.

$$
\beta_{h}=\beta^{*}=\frac{\mu_{v}(\lambda+r-\gamma \alpha)}{n b^{2} \beta_{v}} .
$$

Observe que, ao susbstituirmos o parâmetro, $\beta_{h}=\beta^{*}$ no sistema linearizado (2.70), vamos obter um autovalor nulo de multiplicidade simples. Assim, o Teorema (2.3.3) pode ser usado para analisar a dinâmica do sistema (2.77), próximo de $\beta_{h}=\beta^{*}$.

De fato, ao calcularmos os autovalores da matriz (2.70) em torno de $\beta_{h}=\beta^{*}$, obteremos como autovalores:

$$
\begin{aligned}
& \Lambda_{1}=\Lambda_{2}=-(\lambda-\gamma \alpha)<0 \\
& \Lambda_{3}=0 \\
& \Lambda_{4}=-\left(\lambda+r+\mu_{v}-\gamma \alpha\right)<0 .
\end{aligned}
$$

Além disso é possível verificar que, a matriz jacobiana do sistema (2.77), aplicada ao equilíbrio livre de doença tem um autovetor à direita associado ao autovalor nulo, dado por $v=\left(v_{1}, v_{2}, v_{3}, v_{4}\right)^{T}$, tal que

$$
\left[\begin{array}{cccc}
-(\lambda-\gamma \alpha) & 0 & 0 & -n b \beta^{*} \\
0 & -(\lambda+r-\gamma \alpha) & 0 & n b \beta^{*} \\
0 & r & -(\lambda-\gamma \alpha) & 0 \\
0 & b \beta_{v} & 0 & -\mu_{v}
\end{array}\right] \cdot\left[\begin{array}{l}
v_{1} \\
v_{2} \\
v_{3} \\
v_{4}
\end{array}\right]=\left[\begin{array}{l}
0 \\
0 \\
0 \\
0
\end{array}\right]
$$


o que implica em:

$$
v_{1}=\frac{-n b \beta^{*}}{(\lambda-\gamma \alpha)} v_{4} \quad, \quad v_{2}=\frac{\mu_{v}}{b \beta_{v}} v_{4} \quad, \quad v_{3}=\frac{r \mu_{v}}{b \beta_{v}} v_{4} \quad \text { e } \quad v_{4}=v_{4}>0
$$

Já o autovetor à esquerda, $u=\left(u_{1}, u_{2}, u_{3}, u_{4}\right)^{T}$, associado ao autovalor nulo é dado por:

$$
\left[\begin{array}{cccc}
-(\lambda-\gamma \alpha) & 0 & 0 & 0 \\
0 & -(\lambda+r-\gamma \alpha) & r & b \beta_{v} \\
0 & 0 & -(\lambda-\gamma \alpha) & 0 \\
-n b \beta^{*} & n b \beta^{*} & 0 & -\mu_{v}
\end{array}\right] \cdot\left[\begin{array}{l}
u_{1} \\
u_{2} \\
u_{3} \\
u_{4}
\end{array}\right]=\left[\begin{array}{l}
0 \\
0 \\
0 \\
0
\end{array}\right]
$$

o que implica em:

$$
u_{1}=0 \quad, \quad u_{2}=\frac{\mu_{v}}{n b \beta^{*}} u_{4} \quad, \quad u_{3}=0 \quad \text { e } \quad u_{4}=u_{4}>0
$$

Verificadas as hipóteses do Teorema 2.3.3, vamos obter os coeficientes $a$ e $b$ e, verificarmos qual dos casos é satisfeito pelo sistema (2.77). Sejam

$$
\begin{aligned}
\frac{\partial^{2} f_{1}}{\partial_{x 1} \partial_{x 4}}\left(E_{1}\right) & =\frac{\partial^{2} f_{1}}{\partial_{x 4} \partial_{x 1}}\left(E_{1}\right)=-n b \beta^{*} \quad, \quad \frac{\partial^{2} f_{2}}{\partial_{x 1} \partial_{x 4}}\left(E_{1}\right)=\frac{\partial^{2} f_{2}}{\partial_{x 4} \partial_{x 1}}\left(E_{1}\right)=n b \beta^{*} \quad \mathrm{e} \\
\frac{\partial^{2} f_{4}}{\partial_{x 2} \partial_{x 4}}\left(E_{1}\right) & =\frac{\partial^{2} f_{4}}{\partial_{x 4} \partial_{x 2}}\left(E_{1}\right)=-b \beta_{v}
\end{aligned}
$$

as derivadas parciais com relação ao sistema (2.77), aplicadas ao equilíbrio livre de doença, em $\beta_{h}=\beta^{*}$. Assim, calculando o valor de $a$, temos:

$$
\begin{aligned}
a=\sum_{k, i, j=1}^{4} u_{k} v_{i} v_{j} \frac{\partial^{2} f_{k}\left(E_{1}\right)}{\partial_{x i} \partial_{x j}} & =u_{1} v_{1} v_{4} \frac{\partial^{2} f_{1}\left(E_{1}\right)}{\partial_{x 1} \partial_{x 4}}+u_{2} v_{1} v_{4} \frac{\partial^{2} f_{2}\left(E_{1}\right)}{\partial_{x 1} \partial_{x 4}}+u_{4} v_{2} v_{4} \frac{\partial^{2} f_{4}\left(E_{1}\right)}{\partial_{x 2} \partial_{x 4}} \\
& =-\mu_{v} u_{4} v_{4}^{4}\left[\frac{n b \beta^{*}}{(\lambda-\gamma \alpha)}+1\right]<0
\end{aligned}
$$

pois, o valor entre colchetes é positivo.

Da mesma forma, calcularemos o valor de $b$. Sejam,

$$
\frac{\partial^{2} f_{1}}{\partial_{x 4} \partial_{\phi}}\left(E_{1}\right)=\frac{\partial^{2} f_{1}}{\partial_{\phi} \partial_{x 4}}\left(E_{1}\right)=-n b \quad \text { e } \quad \frac{\partial^{2} f_{2}}{\partial_{x 4} \partial_{\phi}}\left(E_{1}\right)=\frac{\partial^{2} f_{2}}{\partial_{\phi} \partial_{x 4}}\left(E_{1}\right)=n b
$$

as derivadas parciais com relação ao sistema (2.77), em $\beta_{h}=\beta^{*}$. Logo, $b$ é dado por:

$$
\begin{aligned}
b=\sum_{k, i=1}^{4} u_{k} v_{i} \frac{\partial^{2} f_{k}\left(E_{1}\right)}{\partial_{x i} \partial_{\beta^{*}}} & =u_{1} v_{4} \frac{\partial^{2} f_{1}\left(E_{1}\right)}{\partial_{x 4} \partial_{\beta^{*}}}+u_{2} v_{4} \frac{\partial^{2} f_{2}\left(E_{1}\right)}{\partial_{x 4} \partial_{\beta^{*}}} \\
& =\frac{\mu_{v}}{\beta^{*}} u_{4} v_{4}>0 .
\end{aligned}
$$

Portanto, obtivemos $a<0$ e $b>0$. Isso implica que o sistema (2.61) satisfaz o caso 4 do teorema 2.3.3 e portanto, o único equilíbrio endêmico para o sistema (2.61) é localmente assintoticamente estável quando $R_{o}>1$ e $\beta^{*}<\beta_{h}$. Este caso do teorema corresponde a uma bifurcação para frente (forward). 
Finalmente, resta-nos mostrar a estabilidade global do equilíbrio endêmico.

Teorema 2.3.4. Se $R_{o}>1$, então o equilíbrio endêmico $E_{2}=\left(S_{h}^{*}, I_{h}^{*}, R_{h}^{*}, I_{v}^{*}\right)$ é globalmente assintoticamente estável no interior de $\Omega$.

Prova: Conforme observado anteriormente na prova do Teorema 2.3.2, sem perda de generalidade, podemos garantir que as soluções do sistema (2.72) convergem assintoticamente para $E_{2}$ analisando o comportamento do sistema reduzido:

$$
\begin{aligned}
S_{h}^{\prime} & =(\lambda-\gamma \alpha)\left(1-S_{h}\right)-n b \beta_{h} S_{h} I_{v} \\
I_{h}^{\prime} & =n b \beta_{h} S_{h} I_{v}-(\lambda+r-\gamma \alpha) I_{h} \\
I_{v}^{\prime} & =b \beta_{v}\left(1-I_{v}\right) I_{h}-\mu_{v} I_{v} .
\end{aligned}
$$

Além disso, pelo fato de restringirmos nossa análise à um sistema tridimensional, podemos utilizar os mesmos argumentos da teoria de sistemas competitivos para garantir a estabilidade global de $E_{2}$.

De acordo com o teorema 2.1.10, $E_{2}$ é globalmente estável se, verificarmos que o sistema (2.72) é competitivo, persistente e possui a propriedade da estabilidade de órbitas periódicas. Mostraremos essas 3 hipóteses através da prova das seguintes afirmações.

Afirmação 2.3.5. O sistema (2.81) é competitivo.

Prova: Vamos definir a matriz H como sendo:

$$
H=\left[\begin{array}{ccc}
1 & 0 & 0 \\
0 & -1 & 0 \\
0 & 0 & 1
\end{array}\right]
$$

Calculando a matriz jacobiana do sistema (2.81), temos:

$$
J a c=\left[\begin{array}{ccc}
-(\lambda-\gamma \alpha)-n b \beta_{h} I_{v} & 0 & -n b \beta_{h} S_{h} \\
n b \beta_{h} I_{v} & -(\lambda+r-\gamma \alpha) & n b \beta_{h} S_{h} \\
0 & b \beta_{v}\left(1-I_{v}\right) & -b \beta_{v} I_{v}-\mu_{v}
\end{array}\right] .
$$

Calculando $H(J a c) H$, podemos verificar que o sistema (2.81) é competitivo. De fato,

$$
H(D F) H=\left[\begin{array}{ccc}
-(\lambda-\gamma \alpha)-n b \beta_{h} I_{v} & 0 & -n b \beta_{h} S_{h} \\
-n b \beta_{h} I_{v} & -(\lambda+r-\gamma \alpha) & -n b \beta_{h} S_{h} \\
0 & -b \beta_{v}\left(1-I_{v}\right) & -b \beta_{v} I_{v}-\mu_{v}
\end{array}\right]
$$

Assim, podemos concluir que o sistema (2.81) é competitivo em $\Omega$, com relação a ordenação parcial definida pelo octante

$$
K=\left\{\left(S_{h}, I_{h}, I_{v}\right) \in \mathbb{R}^{3}: S_{h} \geq 0, I_{h} \leq 0, I_{v} \geq 0\right\}
$$

Afirmação 2.3.6. O sistema (2.81) é persistente.

Prova: Conforme a proposição 2.1 .13 precisamos mostrar que na fronteira de $\Omega$, o sistema (2.81) tem somente um ponto $\omega$-limite que é o equilíbrio livre de doença, $E_{1}$. Além disso, para $R_{o}>1, E_{1}$ não pode ser $\omega$-limite de qualquer órbita no $\operatorname{int}(\Omega)$. 
Para provarmos a primeira parte, observe que o campo de vetores do sistema (2.81) é transversal na fronteira de $\Omega$ exceto no eixo $S_{h}$ que é invariante pelo fluxo do sistema (2.81). Assim, restritos ao eixo $S_{h}$ temos:

$$
S_{h}^{\prime}(t)=(\lambda-\gamma \alpha)\left(1-S_{h}(t)\right)
$$

Logo, $S_{h} \rightarrow 1$ quando $t \rightarrow \infty$ e com isso concluímos que $E_{1}$ é o único $\omega$-limite no bordo de $\Omega$.

Para verificarmos que, quando $R_{o}>1, E_{1}$ não pode ser $\omega$-limite de qualquer órbita no $\operatorname{int}(\Omega)$, vamos considerar a função de Lyapunov $V:\left\{\left(S_{h}, I_{h}, I_{v}\right) \in \Omega: S_{h}, I_{h}, I_{v}>0\right\} \rightarrow \mathbb{R}$, definida por:

$$
V\left(S_{h}, I_{h}, I_{v}\right)=\left(S_{h}-S_{h}^{*}-S_{h}^{*} \log \left(\frac{S_{h}}{S_{h}^{*}}\right)\right)+\left(I_{h}-I_{h}^{*}-I_{h}^{*} \log \left(\frac{I_{h}}{I_{h}^{*}}\right)\right)+C\left(I_{v}-I_{v}^{*}-I_{v}^{*} \log \left(\frac{I_{v}}{I_{v}^{*}}\right)\right) \geq 0
$$

cujo, $C=\frac{n b \beta_{h} S_{h}^{*} I_{v}^{*}}{b \beta_{v} I_{h}^{*}}$.

Observe que, $V$ é de classe $C^{1}$ no interior de $\Omega$ e $E_{2}$ é um mínimo global de $V$ em $\Omega$. Além disso, $V\left(S_{h}^{*}, I_{h}^{*}, I_{v}^{*}\right)=0$.

Calculando a derivada de $V$ com relação a $t$ e aplicando-a as soluções do sistema (2.81), temos:

$$
\begin{aligned}
V^{\prime} & =\left(1-\frac{S_{h}^{*}}{S_{h}}\right) S_{h}^{\prime}+\left(1-\frac{I_{h}^{*}}{I_{h}}\right) I_{h}^{\prime}+\frac{n b \beta_{h} S_{h}^{*} I_{v}^{*}}{b \beta_{v} I_{h}^{*}}\left(1-\frac{I_{v}^{*}}{I_{v}}\right) I_{v}^{\prime} \\
& =\left(1-\frac{S_{h}^{*}}{S_{h}}\right)\left[(\lambda-\gamma \alpha)\left(1-S_{h}\right)-n b \beta_{h} S_{h} I_{v}\right] \\
& +\left(1-\frac{I_{h}^{*}}{I_{h}}\right)\left[n b \beta_{h} S_{h} I_{v}-(\lambda+r-\gamma \alpha) I_{h}\right] \\
& +\frac{n b \beta_{h} S_{h}^{*} I_{v}^{*}}{b \beta_{v} I_{h}^{*}}\left(1-\frac{I_{v}^{*}}{I_{v}}\right)\left[b \beta_{v}\left(1-I_{v}\right) I_{h}-\mu_{v} I_{v}\right] .
\end{aligned}
$$

Considerando o sistema (2.81) aplicado ao equilíbrio endêmico, $E_{2}=\left(S_{h}^{*}, I_{h}^{*}, I_{v}^{*}\right)$, podemos obter as seguintes relações

$$
(\lambda-\gamma \alpha)=(\lambda-\gamma \alpha) S_{h}^{*}+n b \beta_{h} S_{h}^{*} I_{v}^{*}, \quad(\lambda+r-\gamma \alpha)=n b \beta_{h} S_{h}^{*} \frac{I_{v}^{*}}{I_{h}^{*}} \quad \text { e } \quad \mu_{v}=b \beta_{v}\left(1-I_{v}^{*}\right) \frac{I_{h}^{*}}{I_{v}^{*}} .
$$

Substituindo as relações (2.83) na expressão de $V^{\prime}$ é possível obter:

$$
\begin{aligned}
V^{\prime} & =\left(1-\frac{S_{h}^{*}}{S_{h}}\right)\left[(\lambda-\gamma \alpha) S_{h}^{*}+n b \beta_{h} S_{h}^{*} I_{v}^{*}-(\lambda-\gamma \alpha) S_{h}-n b \beta_{h} S_{h} I_{v}\right] \\
& +\left(1-\frac{I_{h}^{*}}{I_{h}}\right)\left[n b \beta_{h} S_{h} I_{v}-n b \beta_{h} S_{h}^{*} I_{v}^{*} \frac{I_{h}}{\left.I_{h}^{*}\right]}\right. \\
& +\frac{n b \beta_{h} S_{h}^{*} I_{v}^{*}}{b \beta_{v} I_{h}^{*}}\left(1-\frac{I_{v}^{*}}{I_{v}}\right)\left[b \beta_{v}\left(1-I_{v}\right) I_{h}-b \beta_{v}\left(1-I_{v}^{*}\right) I_{h}^{*} \frac{I_{v}}{I_{v}^{*}}\right] \\
& =-(\lambda-\gamma \alpha) \frac{\left(S_{h}-S_{h}^{*}\right)^{2}}{S_{h}}-n b \beta_{h} S_{h}^{*} I_{v}^{*} \\
& {\left[-\left(1-\frac{S_{h}^{*}}{S_{h}}\right)+\left(1-\frac{S_{h}^{*}}{S_{h}}\right) \frac{S_{h} I_{v}}{S_{h}^{*} I_{v}^{*}}-\left(1-\frac{I_{h}^{*}}{I_{h}}\right) \frac{S_{h} I_{v}}{S_{h}^{*} I_{v}^{*}}+\left(1-\frac{I_{h}^{*}}{I_{h}}\right) \frac{I_{h}}{I_{h}^{*}}-\left(1-\frac{I_{v}^{*}}{I_{v}}\right)\left(I_{v}-\frac{I_{h} I_{v}}{I_{h}^{*}}+\frac{I_{h}}{I_{h}^{*}}-\frac{I_{v}}{I_{v}^{*}}\right)\right] } \\
& =-(\lambda-\gamma \alpha) \frac{\left(S_{h}-S_{h}^{*}\right)^{2}}{S_{h}} \\
& -n b \beta_{h} S_{h}^{*} I_{v}^{*}\left[\left(-3+\frac{S_{h}^{*}}{S_{h}}+\frac{I_{h}^{*} S_{h} I_{v}}{I_{h} S_{h}^{*} I_{v}^{*}}+\frac{I_{v}^{*} I_{h}}{I_{v} I_{h}^{*}}\right)-\left(1-\frac{I_{v}^{*}}{I_{v}}\right)\left(I_{v}-\frac{I_{h} I_{v}}{I_{h}^{*}}\right)\right]
\end{aligned}
$$

Desde que, a média aritmética é maior ou igual que a média geométrica, vamos ter: 


$$
\frac{S_{h}^{*}}{S_{h}}+\frac{I_{h}^{*} S_{h} I_{v}}{I_{h} S_{h}^{*} I_{v}^{*}}+\frac{I_{v}^{*} I_{h}}{I_{v} I_{h}^{*}} \geq 3 \quad \text { para todo } \quad S_{h}, I_{h}, I_{v} \geq 0,
$$

e portanto, a expressão entre parênteses é positiva. Sendo assim, resta-nos mostrar que:

$$
I_{v}\left(1-\frac{I_{v}^{*}}{I_{v}}\right)\left(1-\frac{I_{h}}{I_{h}^{*}}\right)<0
$$

e então, concluiremos que, $V^{\prime}\left(S_{h}^{*}, I_{h}^{*}, I_{v}^{*}\right) \leq 0$.

Em particular, a desigualdade (2.84), é verdadeira para os dois casos abaixo:

1) $I_{h}>I_{h}^{*}$ e $I_{v}^{*}<I_{v}$, ou

2) $I_{h}<I_{h}^{*}$ e $I_{v}^{*}>I_{v}$.

Os dois casos acima, são representados pela Figura (2.5)

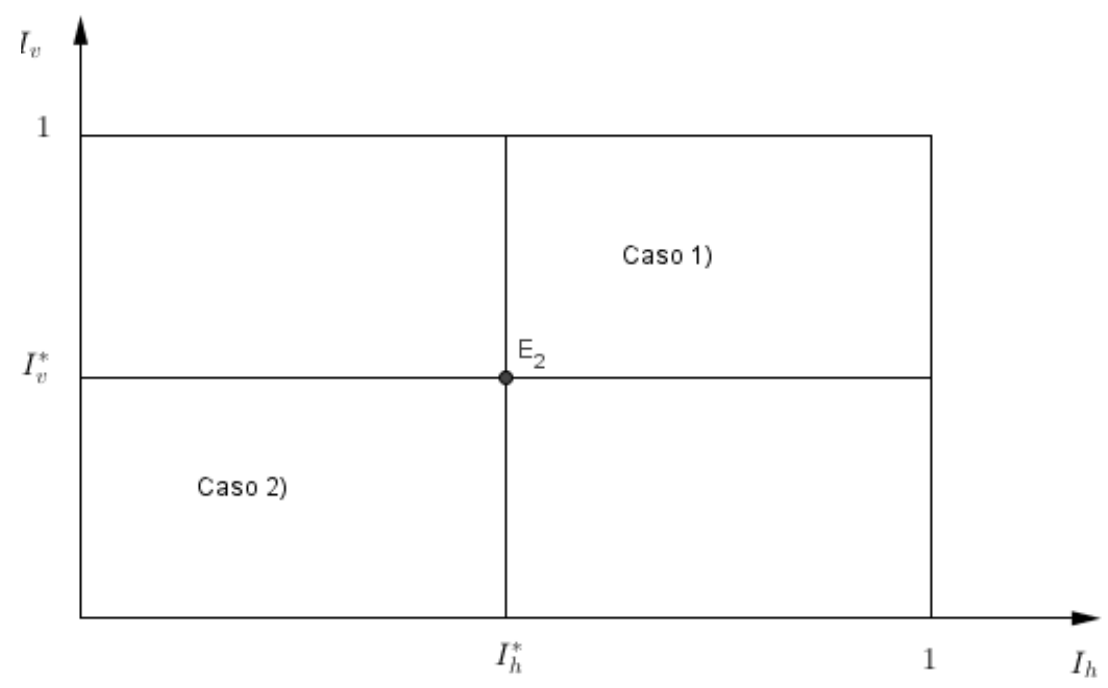

Figura 2.5: Representação gráfica dos casos 1) e 2)

Conforme a Figura (2.5), temos que as órbitas de $\mathrm{V}$ se afastam de $E_{1}$ pois, as soluções do sistema (2.81) para os casos 1 e 2 convergem para o equilíbrio endêmico que é localmente estável. Portanto, para $R_{o}>1, E_{1}$ não pode ser $\omega$-limite de qualquer órbita no $\operatorname{int}(\Omega)$ e assim, concluímos que o sistema (2.81) é persistente.

Afirmação 2.3.7. Qualquer conjunto $\omega$-limite do sistema (2.81), no interior de $\Omega$ é uma órbita fechada ou o equilíbrio endêmico $E_{2}$.

Prova: Suponha que $\gamma$ é um conjunto $\omega$-limite no interior de $\Omega$. Se $\gamma$ não contém $E_{2}$, então $\gamma$ não contém nenhum equilíbrio uma vez que, $E_{2}$ é o único equilíbrio no interior de $\Omega$. Portanto, pelo teorema 2.1.4 um conjunto que não contém equilíbrios é uma órbita fechada. Isso implica que $\gamma$ é uma órbita fechada. Por outro lado, se $\gamma$ contém $E_{2}$, então, para qualquer órbita de $\gamma$ que esteja arbitrariamente próxima de $E_{2}$, convergirá para $E_{2}$ uma vez que, $E_{2}$ é localmente assintoticamente estável. Então $\gamma=E_{2}$.

Por fim, precisamos mostrar que o sistema (2.81) possui a propriedade da estabilidade de órbitas periódicas e então concluiremos a prova do teorema 2.3.4.

Afirmação 2.3.8. O sistema (2.81) tem a propriedade da estabilidade de órbitas periódicas. 
Prova: Conforme descrito pelo Teorema 2.1.9, uma condição suficiente para verificarmos se o sistema (2.81), possui a propriedade da estabilidade de órbitas periódicas, consiste em verificar que o sistema dado por:

$$
\left\{\begin{array}{l}
X^{\prime}=-\left[(\lambda-\gamma \alpha)+n b \beta_{h} I_{v}+(\lambda+r-\gamma \alpha)\right] X+\left(n b \beta_{h} S_{h}\right) Y+\left(n b \beta_{h} S_{h}\right) Z \\
Y^{\prime}=\left(b \beta_{v}\left(1-I_{v}\right)\right) X-\left[(\lambda-\gamma \alpha)+n b \beta_{h} I_{v}+b \beta_{v} I_{v}+\mu_{v}\right] Y \\
Z^{\prime}=\left(n b \beta_{h} I_{v}\right) Y-\left[(\lambda+r-\gamma \alpha)+b \beta_{v} I_{v}+\mu_{v}\right] Z
\end{array}\right.
$$

é assintoticamente estável.

Para isso, usaremos a seguinte função de Lyapunov,

$$
V\left(X(t), Y(t), Z(t), S_{h}(t), I_{h}(t), I_{v}(t)\right)=\sup \left\{|X(t)|, \frac{I_{h}(t)}{I_{v}(t)}(|Y(t)+Z(t)|)\right\}
$$

Uma vez que, o sistema (2.81) é persistente, sabemos que qualquer solução periódica $\left(S_{h}, I_{h}, I_{v}\right)$ está a uma distância positiva do bordo de $\Omega$. Então $\frac{I_{h}(t)}{I_{v}(t)}$ está bem definido e, existe uma constante $0<c<1$ tal que: $\frac{I_{h}(t)}{I_{v}(t)}>c$. Assim, a função $V$ está bem definida ao longo $\left(S_{h}, I_{h}, I_{v}\right)$ e nós temos:

$$
V\left(X, Y, Z, S_{h}, I_{h}, I_{v}\right) \geq c \sup \{|X|,|Y|,|Z|\},
$$

para quaisquer $(X, Y, Z) \in \mathbb{R}^{3}$ e qualquer solução periódica $\left(S_{h}, I_{h}, I_{v}\right)$ do sistema (2.81).

Ao calcularmos a derivada de $V$ ao longo de $(X(t), Y(t), Z(t))$, soluções do sistema (2.85) e $\left(S_{h}, I_{h}, I_{v}\right)$, soluções do sistema (2.81), obtemos as seguintes estimativas:

$$
\begin{gathered}
D_{+}|X(t)| \leq-\left[(\lambda-\gamma \alpha)+n b \beta_{h} I_{v}+(\lambda+r-\gamma \alpha)\right]|X(t)|+n b \beta_{h} S_{h}(|Y(t)|+|Z(t)|) \\
\leq-\left[(\lambda-\gamma \alpha)+n b \beta_{h} I_{v}+(\lambda+r-\gamma \alpha)\right]|X(t)|+n b \beta_{h} S_{h} \frac{I_{v}}{I_{h}}\left(\frac{I_{h}}{I_{v}}(|Y(t)|+|Z(t)|)\right), \\
D_{+}|Y(t)| \leq b \beta_{v}\left(1-I_{v}\right)|X(t)|-\left[(\lambda-\gamma \alpha)+n b \beta_{h} I_{v}+b \beta_{v} I_{v}+\mu_{v}\right]|Y(t)|, \\
D_{+}|Z(t)| \leq n b \beta_{h} I_{v}|Y(t)|-\left[(\lambda-\gamma \alpha)+b \beta_{v} I_{v}+\mu_{v}\right]|Z(t)| .
\end{gathered}
$$

Somando-se as desigualdades (2.89) e (2.90), vamos obter:

$$
D_{+}[|Y(t)|+|Z(t)|] \leq b \beta_{v}\left(1-I_{v}\right)|X(t)|-\left[(\lambda-\gamma \alpha)+b \beta_{v} I_{v}+\mu_{v}\right](|Y(t)|+|Z(t)|),
$$

e portanto,

$$
\begin{aligned}
D_{+}\left[\frac{I_{h}}{I_{v}}|Y(t)|+|Z(t)|\right] & =\left(\frac{I_{h}^{\prime}}{I_{h}}-\frac{I_{v}^{\prime}}{I_{v}}\right) \frac{I_{h}}{I_{v}}(|Y(t)|+|Z(t)|)+\frac{I_{h}}{I_{v}} D_{+}[|Y(t)|+|Z(t)|] \\
& \leq \frac{I_{h}}{I_{v}} b \beta_{v}\left(1-I_{v}\right)|X(t)|+\frac{I_{h}}{I_{v}}\left(\frac{I_{h}^{\prime}}{I_{h}}-\frac{I_{v}^{\prime}}{I_{v}}-(\lambda-\gamma \alpha)-b \beta_{v} I_{v}-\mu_{v}\right)(|Y(t)|+|Z(t)|) .
\end{aligned}
$$

Das desigualdades, (2.88) e (2.91),segue:

$$
D_{+} V(t) \leq \sup \left\{g_{1}(t), g_{2}(t)\right\} V(t)
$$

onde, 


$$
\begin{gathered}
g_{1}(t)=-\left[(\lambda-\gamma \alpha)+n b \beta_{h}+(\lambda+r-\gamma \alpha)\right]+n b \beta_{h} S_{h} \frac{I_{v}}{I_{h}} \\
g_{2}(t)=\frac{I_{h}}{I_{v}} b \beta_{v}\left(1-I_{v}\right)+\frac{I_{h}^{\prime}}{I_{h}}-\frac{I_{v}^{\prime}}{I_{v}}-(\lambda-\gamma \alpha)-b \beta_{v} I_{v}-\mu_{v} .
\end{gathered}
$$

Do sistema (2.81), nós temos:

$$
\frac{I_{v}^{\prime}}{I_{v}}=b \beta_{v}\left(1-I_{v}\right) \frac{I_{h}}{I_{v}}-\mu_{v} .
$$

Substituindo a equação (2.95) em (2.94):

$$
g_{2}(t)=\frac{I_{h}^{\prime}}{I_{h}}-(\lambda-\gamma \alpha)-b \beta_{v} I_{v} .
$$

Similarmente $\frac{I_{h}^{\prime}}{I_{h}}=n b \beta_{h} S_{h} \frac{I_{v}}{I_{h}}-(\lambda+r-\gamma \alpha)$. Então

$$
g_{1}(t)=\frac{I_{h}^{\prime}}{I_{h}}-(\lambda-\gamma \alpha)-n b \beta_{h} .
$$

Assim,

$$
\sup \left\{g_{1}(t), g_{2}(t)\right\} \leq \frac{I_{h}^{\prime}}{I_{h}}-(\lambda-\gamma \alpha) .
$$

Da equação (2.92) e pela desigualdade de Gronwall A.2.10 (ver apêndice A.1),

$$
V(t) \leq V(0) I_{h}(t) e^{(\lambda-\gamma \alpha) t} \leq V(0) e^{-(\lambda-\gamma \alpha) t}
$$

o que implica, $V(t) \rightarrow 0$ quando $t \rightarrow \infty$ pois, $(\lambda-\gamma \alpha)>0$. Consequentemente, o sistema (2.85) é assintoticamente estável e portanto, a solução periódica $\left(S_{h}, I_{h}, I v\right)$ é assintoticamente e orbitalmente estável. Isso conlui a prova da afirmação.

Com a prova das afirmações acima, verificamos as hipóteses do Teorema 2.1.10 e por conseguinte, este resultado garante que, se $R_{o}>1$, o equilíbrio endêmico, $E_{2}$ é globalmente assintoicamente estável.

\subsection{Comentários}

Neste capítulo, desenvolvemos um estudo qualitativo para um modelo determinístico de propagação de dengue do tipo SIR com acoplamento da dinâmica do vetor. Três hipóteses sobre o tamanho total da população humana foram avaliadas e correspondem respectivamente, ao modelo 1 (população humana constante ao longo do tempo), modelo 2 (população humana com crescimento exponencial) e modelo 3 (população humana com crescimento logístico). O modelo matemático incorpora uma taxa de morte causada pela doença somente no caso em que consideramos uma função de crescimento exponencial para a população humana. O mesmo não admite transmissão vertical e a taxa de transmissão da doença é considerada dependente das densidades de humanos e vetores.

O comportamento global das soluções para cada um dos modelos matemáticos foi analisado em função das frações das populações $\left(S_{h}, I_{h}, R_{h}, S_{v}, I_{v}\right)$. Pelo fato do equilíbrio endêmico ser uma medida que reflete a prevalência da doença nos preocupamos em verificar condições que garantam a estabilidade global deste equilíbrio. Para isso, o parâmetro $R_{o}$ foi calculado conforme a metodologia descrita na Seção 1.4.5.

A partir do cálculo do $R_{o}$ vimos que este parâmetro depende exclusivamente dos parâmetros de cada modelo e da variável $N_{h}$. No caso do modelo 3, cuja população humana é dada por uma função de crescimento logístico, o parâmetro $R_{o}$ é inversamente proporcional a variável $N_{h}$. Dessa forma, considerando todos os parâmetros do modelo fixos, este parâmetro diminui conforme o tamanho da população humana aumenta. 
No caso do modelo 2, cuja população apresenta um crescimento exponencial, o valor do parâmetro $R_{o}$ é fixo dado que a expressão de $R_{o}$ envolve somente os parâmetros deste modelo. O mesmo ocorre com o modelo 1. Apesar da expressão para o parâmetro $R_{o}$ depender da variável $N_{h}$, neste caso a variável correspondente a população humana é mantida fixa.

Com o parâmetro $R_{o}$ foram obtidos as expressões para os equilíbrios livre de doença, $E_{1}$, e equilíbrio endêmico, $E_{2}$. Os resultados do estudo de estabilidade para os equilíbrios, referentes aos três modelos, foram os seguintes:

i) O equilíbrio livre de doença é localmente assintoticamente estável quando a quantidade limiar, $R_{o}$, é menor que uma unidade. Esta estabilidade local para o equilíbrio livre de doença foi verificada para os modelos 1 e 3 através do critério de Routh-Hurwitz enquanto que para o modelo 2 foi usada uma propriedade de sistema competitivos tridimensionais;

ii) Para o modelo 2 a existência e unicidade do equilíbrio endêmico é garantida pelas condições $\nu_{h}>\alpha_{h}$ e $\gamma_{h}>\alpha_{h}$

iii) Para o modelo 3 verificamos que em $R_{o}=1$ o modelo apresenta uma bifurcação transcrítica. Essa bifurcação é forward e o equilíbrio endêmico existe e é localmente assintoticamente estável quando este limiar atravessa a unidade, ou seja, $R_{o}>1$;

iv) O equilíbrio livre de doença é globalmente estável para $R_{o} \geq 1$. A estabilidade global foi verificada através da construção de uma função de Lyapunov e pelo teorema da Invariânica de Lasalle;

v) O equilíbrio livre de doença é instável para $R_{o}>1$;

vi) O equilíbrio endêmico é globalmente assintoticamente estável para $R_{o}>1$. A estabilidade global foi verificada usando um critério geral para a estabilidade de órbitas periódicas de sistemas autônomos não lineares de altas ordens, assim como argumentos da teoria de sistemas competitivos para equações diferenciais. 


\section{Capítulo 3}

\section{Resultados Numéricos}

No capítulo anterior, analisamos a formulação de três modelos de propagação da dengue em termos das proporções de humanos e vetores, considerando diferentes hipóteses sobre o crescimento da população total de humanos. Mostramos que o equilíbrio endêmico desses modelos quando existe, é localmente e globalmente assintoticamente estável.

Neste capítulo, temos como objetivos: validar os resultados teóricos com técnicas numéricas, avaliar qualitativamente o comportamento dos modelos ao longo de longo períodos, identificar a influência da modelagem das variações populacionais nas análises de longo termo e por fim identificar os parâmetros mais sensíveis de cada modelo através de uma análise de sensibilidade do parâmetro $R_{o}$ e do equilíbrio endêmico. Iniciaremos descrevendo as técnicas numéricas que serão utilizadas. Em seguida, apresentaremos os resultados obtidos para os pontos de equilíbrio, bem como a solução numérica dos modelos. Uma discussão sobre o comportamento do parâmetro $R_{o}$ com relação a variação nas hipóteses sobre o crescimento da população humana (constante, crescimento exponencial e crescimento logístico) será abordada. Por fim serão apresentados os resultados da análise de sensibilidade.

\subsection{Métodos numéricos}

As soluções numéricas dos modelos matemáticos de propagação de dengue serão feitas pelo método Rosenbrock de terceira ordem e quatro estágios, ROS34, pelo fato dos sistemas de equações diferenciais ordinárias estudados serem rígidos. Em geral, não existe uma única definição para rigidez. Para Hairer e Wanner, em [WH91], equações rígidas são problemas que métodos explícitos não trabalham bem. Conforme os autores, problemas rígidos são encontrados comumente em reações químicas, circuitos elétricos, difusão, entre outros. Em epidemiologia particularmente, este fenômeno é observado em modelos compartimentais com retardo. O retardo é utilizado nestes modelos para representar a duração do período infeccioso, do período imune, latência, ou duração da imunidade temporária.

Estes modelos são considerados rígidos no sentido que se tornam lentos e caros para resolvê-los usando métodos explícitos. Embora o modelo em estudo não apresente retardo esta característica pode ser facilmente incluída ao modelo, uma vez que a dengue apresenta essa característica. Portanto, no caso dos modelos de epidemiologia vamos utilizar a seguinte definição para rigidez ([CH52]):

Definição 3.1.1. Equações diferenciais que descrevem um problema rígido impõem dificuldades às técnicas numéricas explícitas normalmente utilizadas.

O método Rosenbrock é um método numérico de passo único construído a partir da linearização de métodos Runge-Kutta implícitos. Esse método substitui a resolução de sistemas não lineares pela resolução de uma sequência de sistemas lineares o que facilita o processo de implementação do método. Optamos pela escolha deste método em detrimento a métodos clássicos, como Runge-Kutta44, pois este método além de ser adequado para trabalhar com problemas rígidos trabalha com uma fórmula embutida de erro para 
controle do passo de integração. A fórmula embutida de controle de passo torna esse método mais eficiente computacionalmente do que o método Runge-Kutta44 por exemplo, que trabalha com um passo de integração fixo.

Para uma revisão mais detalhada sobre convergência, estabilidade, detalhes sobre a implementação do método ROS34, bem como outras aplicações deste método consultar o trabalho de Sartori, em [Sar14] e Hairer, em [HNW10].

Para grande parte dos modelos em epidemiologia, a obtenção de expressões analíticas para o equilíbrio endêmico não é possível, pois as equações dos modelos em geral envolvem muitas não linearidades. Dessa forma, optamos pela implentação de um método numérico para obtenção destes equilíbrios. O método de Broyden foi escolhido dentro da classe de métodos Quase-Newton. A motivação central dos métodos QuaseNewton é gerar uma sequência $\left\{x_{k}\right\}, k=1, \cdots, n$, com boas propriedades de convergência, sem no entanto avaliar a matriz jacobiana a cada iteração, como é necessário no método de Newton ([SB13]). No caso dos modelos 1, 2 e 3 foram usadas como condições iniciais para o método de Broyden a solução numérica de cada modelo. Os resultados para os equilíbrios endêmicos são dados pela Tabela 3.1:

\begin{tabular}{lll}
\hline Modelo 1 & Modelo 2 & Modelo 3 \\
\hline$S_{h}^{*}=0.0703$ & $S_{h}^{*}=0.1384$ & $S_{h}^{*}=0.1407$ \\
$I_{h}^{*}=0.00026$ & $I_{h}^{*}=0.0108$ & $I_{h}^{*}=0.00009$ \\
$I_{v}^{*}=0.00037$ & $I_{v}^{*}=0.0153$ & $R_{h}^{*}=0.8592$ \\
- & - & $I_{v}^{*}=0.000128$ \\
\hline
\end{tabular}

Tabela 3.1: Proporções endêmicas para cada modelo.

\subsection{Comportamento assintótico}

Para explorarmos o comportamento assintótico das soluções dos modelos matemáticos (2.3), (2.33), (2.61) e verificarmos numericamente a estabilidade global do equilíbrio endêmico foram selecionados parâmetros teóricos relativos a dengue. O critério de seleção incluiu a escolha de valores consagrados pela comunidade acadêmica da área que atendessem as hipóteses dos modelos estudados. A Tabela 3.2 sumariza os valores dos parâmetros que serão usados em todas as simulações para cada modelo.

\begin{tabular}{lllll}
\hline Parâmetro & Modelo1 & Modelo2 & Modelo3 & Referência \\
\hline$b$ & $1 \mathrm{dia}^{-1}$ & - & $1 \mathrm{dia}^{-1}$ & [NR92] \\
$\beta_{h}, \lambda_{h}$ & 0.75 & 0.75 & 0.75 & [NR92] \\
$\beta_{v}, \lambda_{v}$ & 0.375 & 0.375 & 0.375 & [NR92] \\
$\mu_{h}, \nu_{h}, \lambda$ & $0.00004 \mathrm{dia}^{-1}$ & $0.00184 \mathrm{dia}^{-1}$ & $0.00004 \mathrm{dia}^{-1}$ & [NS00] e [dGeEI] \\
$\mu_{h}$ & $0.00004 \mathrm{dia}^{-1}$ & $0.000015 \mathrm{dia}^{-1}$ & $0.000015 \mathrm{dia}^{-1}$ & [dGeEI] \\
$k$ & - & - & 20000 & Escolhido \\
$\alpha_{h}$ & - & $0.001 \mathrm{dia}^{-1}$ & - & [CBLM06] \\
$\gamma_{h}, r$ & $0.143 \mathrm{dia}^{-1}$ & $0.143 \mathrm{dia}^{-1}$ & $0.143 \mathrm{dia}^{-1}$ & [CBLM06] \\
$m$ & 0 & - & - & [EV98] \\
$N_{h}$ & 10000 & 10000 & 10000 & [EV98] \\
$\mu_{v}$ & $0.263 \mathrm{dia}^{-1}$ & $0.263 \mathrm{dia}^{-1}$ & $0.263 \mathrm{dia}^{-1}$ & [CBLM06] \\
$A, D$ & 5000 & - & 5000 & [EV98] \\
\hline
\end{tabular}

Tabela 3.2: Valores dos parâmetros de cada modelo utilizados nas simulações.

Embora nossa análise considere todos os parâmetros fixos, é importante ressaltar que esses parâmetros não são estáticos a longo prazo e podem apresentar dependência de outros fatores. O trabalho de Massad 
et al., em [MF98], evidencia essa dependência modelando a sensibilidade de parâmetros de significância epidemiológica com relação a temperatura.

O período usado para as simulações foi definido em 40 anos para que pudéssemos identificar a influência da modelagem das variações populacionais e ainda verificar a estabilidade assintótica dos equilíbrios endêmicos. No tempo $t=0$, utilizamos como condições iniciais os valores apresentados na Tabela 3.3.

\begin{tabular}{lll}
\hline Modelo 1 & Modelo 2 & Modelo 3 \\
\hline$S_{h}(0)=0.25$ & $S_{h}(0)=0.25$ & $S_{h}(0)=0.25$ \\
$I_{h}(0)=0.1$ & $I_{h}(0)=0.1$ & $I_{h}(0)=0.1$ \\
$I_{v}(0)=0.02$ & $I_{v}(0)=0.02$ & $R_{h}(0)=0.65$ \\
- & - & $I_{v}(0)=0.02$ \\
\hline
\end{tabular}

Tabela 3.3: Condições iniciais utilizadas para as simulações.

O método implementado foi testado para diferentes conjuntos de condições iniciais, entretanto o comportamento qualitativo manteve-se praticamente o mesmo para todos os testes. As Figuras 3.1, 3.3 e 3.2 refletem o comportamento qualitativo das soluções dos modelos 1, 3 e 2 respectivamente, no decorrer de 40 anos.

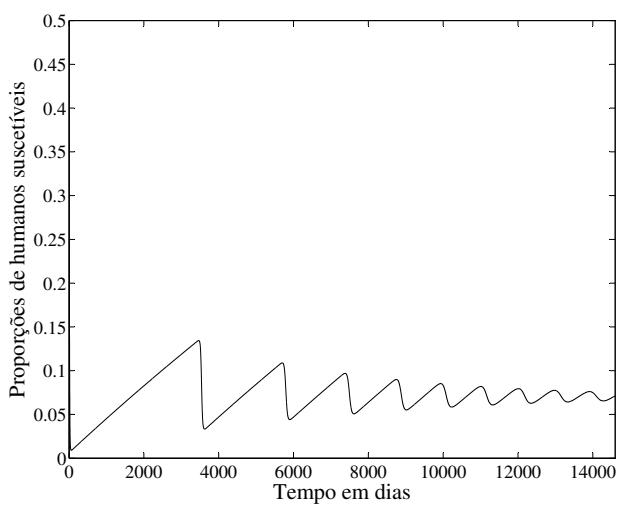

(a) Proporção de humanos suscetíveis ao longo de 40 anos

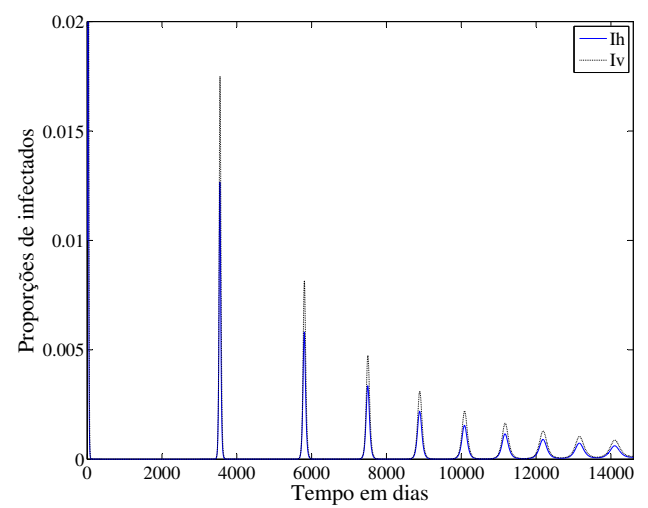

(b) Proporções de infectados ao longo de 40 anos

Figura 3.1: Solução numérica do modelo 1 avaliada durante o período de 40 anos. 


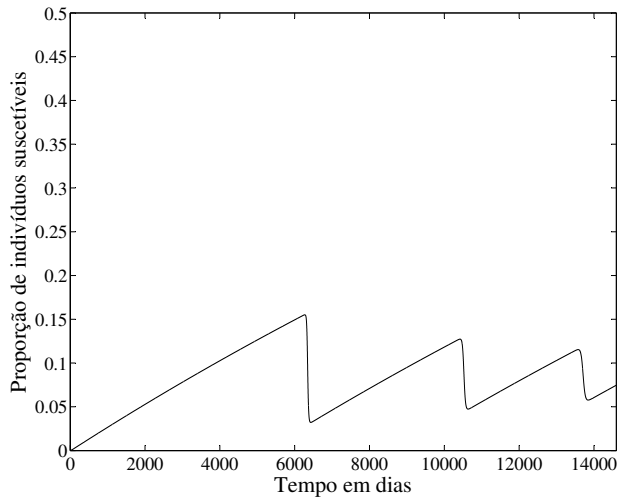

(a) Proporção de humanos suscetíveis ao longo de 40 anos

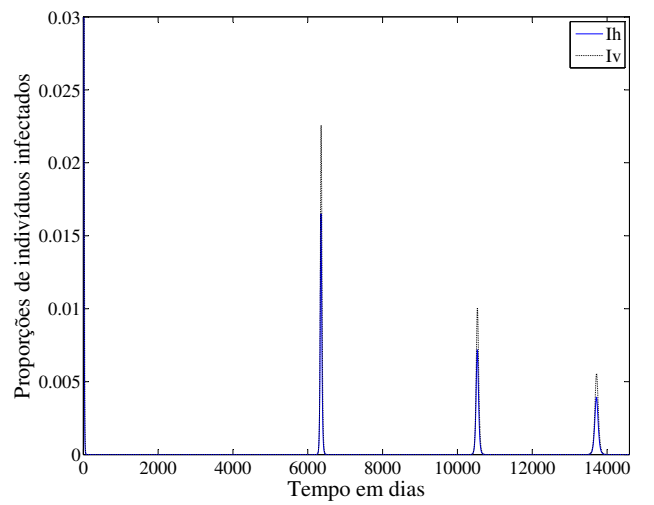

(b) Proporções de infectados ao longo de 40 anos

Figura 3.2: Solução numérica do modelo 3 avaliada durante o período de 40 anos.

Os modelos 1 e 3 apresentam um comportamento qualitativo semelhante exceto pela velocidade da dinâmica. O modelo 3 apresenta uma dinâmica mais lenta devido a hipótese sobre o crescimento da população humana ser dada por uma função de crescimento logístico. A entrada de novos suscetíveis no sistema torna a dinâmica de contatos entre suscetíveis e infectados mais lenta. O modelo 1, por outro lado, ao considerar a população constante permite que os contatos entre suscetíveis e infectados saturem-se mais rapidamente pela falta de entrada de novos suscetíveis.

As soluções de ambos os modelos oscilam para as proporções do equilíbrio endêmico. Dado que a solução numérica desses modelos (Figuras 3.1, 3.2, respectivamente) representa o cenário endêmico, temos neste caso $R_{o}>1$. Além disso, como a expectativa de vida de humanos é em torno de 76 anos, o parâmetro $\mu_{h}$ é muito menor do que 1. Essas duas observações juntas implicam que as soluções dos modelos 1 e 3 oscilem para as proporções no equilíbrio endêmico.

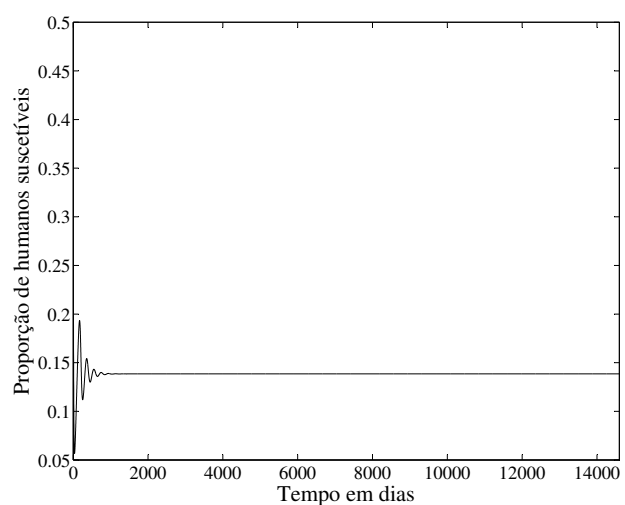

(a) Proporção de humanos suscetíveis ao longo de 40 anos

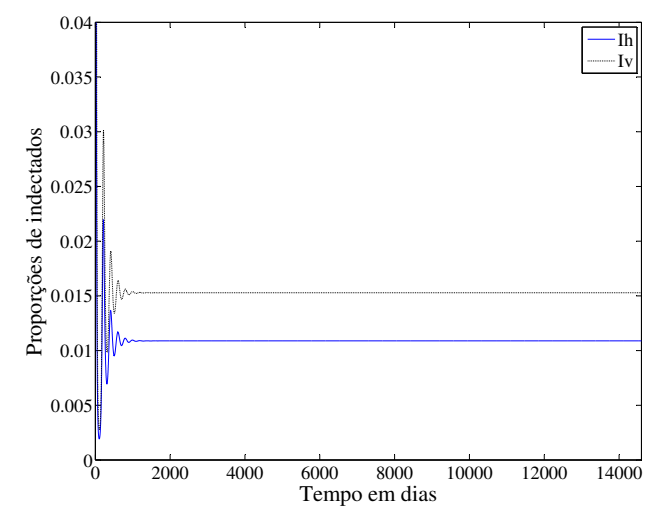

(b) Proporções de infectados ao longo de 40 anos

Figura 3.3: Solução numérica do modelo 2 avaliada durante o período de 40 anos.

A dinâmica das soluções do modelo 2 difere essencialmente pelas hipóteses que restringem a existência e unicidade do equilíbrio endêmico, $\nu_{h}>\alpha_{h}$ e $\gamma_{h}>\alpha_{h}$. Pelo fato de considerarmos $\alpha_{h}=0.001$, para que a hipótese $\nu_{h}>\alpha_{h}$ seja satisfeita consideramos $\nu_{h}=0.00184$, o que em termos dimensionais corresponde a aproximadamente a taxa per capita de 28 nascimentos a cada 1000 pessoas por ano ([NS00]). Neste caso, o modelo apresenta algumas oscilações no início do período analisado, porém essas oscilações estabilizam-se 
rapidamente convergindo assintoticamente para o equilíbrio endêmico.

Outra maneira de visualizarmos a convergência das soluções dos modelos para seus respectivos equilíbrios é através do retrato de fase. A Figura 3.4 apresenta o retrato de fase de cada um dos modelos.

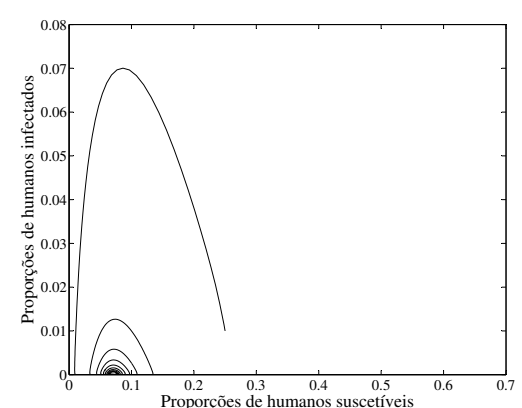

(a) Retrato de fase do modelo 1

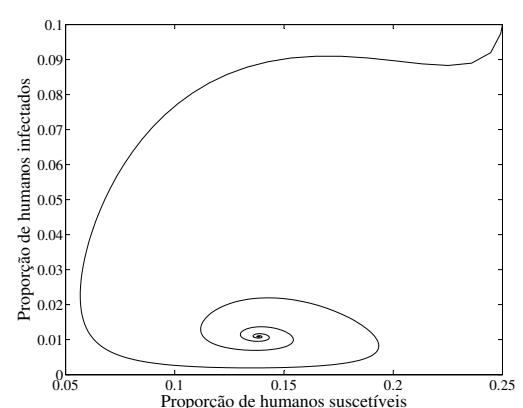

(b) Retrato de fase do modelo 2

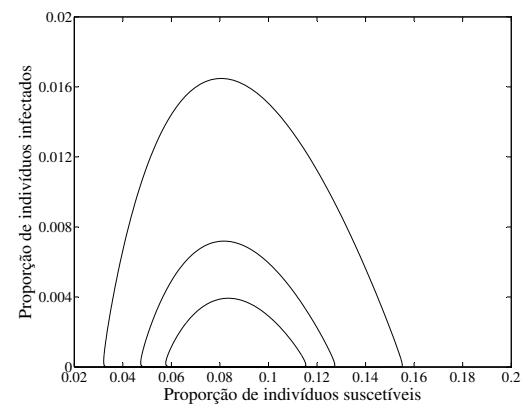

(c) Retrato de fase do modelo 3

Figura 3.4: Retrato de fase dos modelos 1,2 e 3 .

\subsection{Parâmetro $R_{o}$}

No capítulo 2, foram obtidas as expressões analíticas para o parâmetro $R_{o}$. Para os modelos 1 e 2 o valor de $R_{o}$ é constante, pois este limiar depende única e exclusivamente dos parâmetros desses modelos. No caso do modelo 3 , o parâmetro $R_{o}$, além da dependência dos parâmetros apresenta variação no tempo. Abaixo apresentamos as estimativas do parâmetro $R_{o}$ para os modelos 1,2 e 3 considerando os parâmetros descritos na Tabela 3.2:

$$
\text { a) } \quad R_{o}=14.2132 \quad \text { b) } \quad R_{o}=7.3326 \quad \text { c) } \quad R_{o}(t)=\frac{D b^{2} \beta_{h} \beta_{v}}{\mu_{v}^{2} N_{h}(t)\left(\lambda+r-\gamma \frac{N_{h}(t)}{k}\right)} .
$$

A Figura 3.5 apresenta a variação no parâmetro $R_{o}$, referente ao modelo 3 , com relação ao tempo.

Ao obtermos as estimativas para este parâmetro observou-se que os modelos 1 e 3 no instante inicial são praticamente iguais. Entretanto, como estamos considerando um período de estudo relativamente longo, a hipótese sobre o crescimento da população faz com este parâmetro, relativamente ao modelo 3 , decresça com o passar do tempo. O modelo 2 apresenta uma das menores estimativas porém não considera o crescimento da população. 


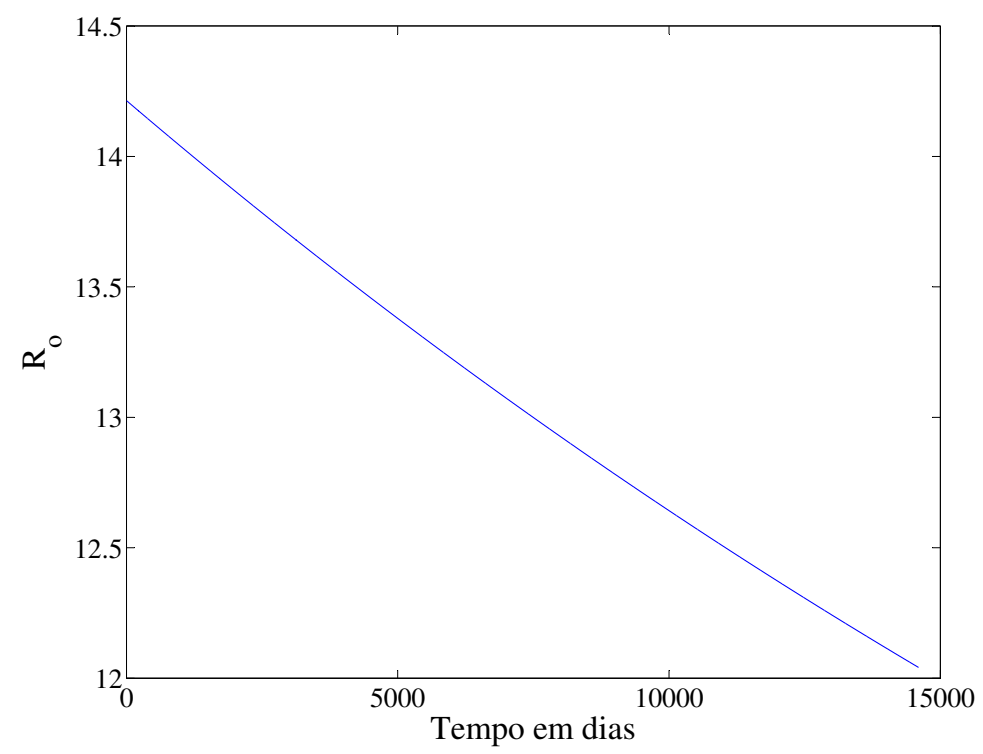

Figura 3.5: Variação do parâmetro $R_{o}$ com relação ao tempo.

\subsection{Análise de sensibilidade}

Para determinarmos a melhor forma de reduzir o número de infectados de determinada doença, é necessário conhecer a importância relativa de diferentes fatores responsáveis pela transmissão e prevalência da doença. A transmissão inicial da doença está relacionada diretamente ao parâmetro $R_{o}$ enquanto que o equilíbrio endêmico está relacionado a prevalência da doença, mais precisamente a magnitude das variáveis de estado.

Nesta subseção faremos duas análises de sensibilidade. A primeira será uma análise de sensibilidade do parâmetro $R_{o}$ com relação aos parâmetros e a segunda análise de sensibilidade, será do equilíbrio endêmico com relação aos parâmetros. Todos os parâmetros serão usados nesta análise.

\subsubsection{Descrição da análise de sensibilidade}

Incertezas com relação aos parâmetros são inevitáveis, desde que grande parte dos parâmetros não podem ser medidos diretamente. Neste contexto, a análise de sensibilidade surge como uma ferramenta que permite determinar quais parâmetros são mais sensíveis a ponto de mudar o comportamento qualitativo das soluções dos modelos de propagação. Além disso, o conhecimento dos parâmetros que acarretam em mudanças mais significativas podem ser usados como medidas de controle e mais do que isso, podem reforçar a importância da coleta de dados e acurácia na medição dessas informações.

O índice de sensibilidade permite-nos medir mudanças relativas as variáveis de estado dos modelos com relação a pequenas mudanças nos valores dos parâmetros. Usaremos a definição de índice de sensibilidade apresentada no trabalho de Chitnis et al., em [CHC08].

Definição 3.4.1. O indice de sensibilidade normalizado para frente, da variável u, que depende diferencialmente do parâmetro $p$ é definido como:

$$
\Gamma_{p}^{u}:=\frac{\partial u}{\partial p} \times \frac{p}{u}
$$

A normalização é feita para que parâmetros em diferentes escalas de tempo possam ser comparados. Esta definição também é conhecida como análise de elasticidade. 


\subsection{2 Índices de sensibilidade para o $R_{o}$}

Dado que o parâmetro $R_{o}$ mede a transmissão inicial da doença, vamos discutir nesta subseção quais parâmetros são mais sensíveis a perturbações locais e como essas perturbações podem impactar na transmissão inicial da doença. Como obtivemos uma expressão explícita para $R_{o}$, para cada um dos modelos, (ver expressões (2.4), (2.34) e (2.63)), aplicaremos a Definição 3.4.1.

Por exemplo, o índice de sensibilidade do $R_{o}$, associado ao modelo 1 , com relação a taxa de picadas, $b$, é dado por:

$$
\Gamma_{p}^{R_{o}}:=\frac{\partial R_{o}}{\partial b} \times \frac{b}{R_{o}}=+1
$$

A Tabela 3.4, sumariza os resultados dos índices de sensibilidade para $R_{o}$ para os três modelos. Esses índices foram avaliados considerando os parâmetros descritos na Tabela 3.2.

\begin{tabular}{|c|c|c|c|c|c|}
\hline \multicolumn{2}{|c|}{$R_{o}$ Modelo 1} & \multicolumn{2}{|c|}{$R_{o}$ Modelo 2} & \multicolumn{2}{|c|}{$R_{o}$ Modelo 3} \\
\hline Parâmetro & Îndice & Parâmetro & Îndice & Parâmetro & Îndice \\
\hline$b$ & +1 & $\lambda_{h}$ & +0.5 & $b$ & +1 \\
\hline$\mu_{v}$ & -1 & $\lambda_{v}$ & +0.5 & $\mu_{v}$ & -1 \\
\hline$\beta_{v}$ & +0.5 & $\mu_{v}$ & -0.5 & $\beta_{h}$ & +0.5 \\
\hline$A$ & +0.5 & $\gamma_{h}$ & -0.49 & $\beta_{v}$ & +0.5 \\
\hline$\beta_{h}$ & +0.5 & $\nu_{h}$ & -0.0063 & $D$ & +0.5 \\
\hline$\gamma_{h}$ & -0.5 & $\alpha_{h}$ & -0.0033 & $r$ & -0.49 \\
\hline$\mu_{h}$ & -0.00014 & & & $\lambda$ & -0.000069 \\
\hline & & & & $\mu_{h}$ & -0.0000262 \\
\hline & & & & $k$ & -0.0000437 \\
\hline
\end{tabular}

Tabela 3.4: Índices de sensibilidade do $R_{o}$ com relação ao valor dos parâmetros de cada modelo que estão descritos na Tabela 3.2.

Ao calcularmos o índice de sensibilidade do $R_{o}$ com relação aos parâmetros de cada modelo, observamos que alguns desses índices não dependem diretamente do valor do respectivo parâmetro. De forma geral, os índices de sensibilidade calculados com relação a parâmetros que envolvem a dinâmica do vetor, taxas de contato entre suscetíveis e infectados, independem do valor atribuído a estes parâmetros. Por exemplo:

$$
\Gamma_{\beta_{v}}^{R_{o}}:=\frac{\partial R_{o}}{\partial \beta_{v}} \times \frac{\beta_{v}}{R_{o}}=+0.5
$$

Esta observação sugere uma atenção especial para estes parâmetros, pois o índice de sensibilidade neste caso é inerente ao parâmetro e não ao valor que este parâmetro pode assumir. Por outro lado, o índice de sensibilidade dos parâmetros demográficos depende do valor assumido para estes parâmetros. Por exemplo:

$$
\Gamma_{\mu_{h}}^{R_{o}}:=\frac{\partial R_{o}}{\partial \mu_{h}} \times \frac{\mu_{h}}{R_{o}}=\frac{-1}{2} \frac{\mu_{h}}{\mu_{h}+\gamma_{h}} .
$$

Neste caso, dependendo do intervalo de definição considerado para cada parâmetro, teremos uma variação na aplitude dos valores dos índices de sensibilidade.

Através da cálculo dos índices de sensibilidade do parâmetro $R_{o}$, propomos uma divisão destes índices em três blocos: bloco 1) serão considerados os parâmetros de alto impacto, cujo índice de sensibilidade é maior do 0.5. No bloco 2), serão considerados parâmetros de médio impacto, cujo índice de sensibilidade está entre 0.4 e 0.5. No bloco 3 serão considerados parâmetros de baixo impacto, cujo índive de sensibilidade está abaixo de 0.4. O sinal positivo para o índice de sensibilidade indica que o parâmetro é diretamente proporcional ao $R_{o}$, enquanto que o sinal negativo para o índice de sensibilidade indica que o parâmetro é inversamente proporcional ao $R_{o}$.

Dentre os parâmetros de alto impacto estão a taxa de picadas do vetor, $b$ e a taxa de morte do vetor, $\mu_{v}$. Os parâmetros desse bloco foram mais expressivos para os valores de $R_{o}$, referentes aos modelos 1 e 
3. Decrescendo o parâmetro $b$ em $1 \%$ vamos ter uma decréscimo do parâmetro $R_{o}$ de $1 \%$. Caso contrário, aumentando o parâmetro $b$ em $1 \%$ vamos ter um aumento no parâmetro $R_{o}$ de $1 \%$.

Os parâmetros de médio impacto correspondem as taxas de transmissão de humano para vetor, $\lambda_{v}, \beta_{v}$, transmissão de vetor para humanos, $\lambda_{h}, \beta_{h}$, a taxa de recrutamento de vetores, $A, D$ e a taxa de recuperação de humanos, $\gamma_{h}, r$. Esses parâmetros de médio impacto são evidenciados por todos os modelos. Variando estes parâmetros em $1 \%$ vamos ter uma aumento ou decréscimo do parâmetro $R_{o}$ em torno de $0,5 \%$. Exceto pela taxa de recuperação de humanos, $\gamma_{h}, r$, os parâmetros do bloco 2) são diretamente proporcionais ao $R_{o}$.

Dentre os parâmetros de baixo impacto estão os parâmetros demográficos, como a taxa de morte de humanos, $\mu_{h}$, taxa de nascimento de humanos, $\nu_{h}, \lambda$ e a taxa de morte pela doença, $\alpha_{h}$. Apesar desses parâmetros obterem um índice de sensibilidade menor do que os demais eles podem assumir uma certa variabilidade dependendo das características demográficas de cada região.

Duas considerações importantes podem ser destacadas desta análise. A primeira delas corresponde a identificação de parâmetros que apresentem possibilidades teóricas de controle. A taxa de picadas pode ser reduzida evitando-se o contato entre humanos e mosquitos através de telas de proteção e o uso de repelentes, por exemplo. A taxa de mortalidade de mosquitos pode ser aumentada através da introdução de inseticidas ou da eliminação possíveis focos da doença ainda na fase aquática.

A segunda observação relaciona-se ao valor do parâmetro $R_{o}$. O cenário abordado neste capítulo corresponde ao cenário endêmico, com $R_{o}>1$. Nesta análise, vimos que os parâmetros de alto impacto contribuem com variações significativas no valor de $R_{o}$. Nesse sentido, um cenário complicado seriam valores de $R_{o}$ muito próximos de 1 , onde uma pequena variação nos parâmetros deste bloco poderia ocasionar uma mudança de estado, onde sairíamos de uma situação sem doença para outra com doença.

\subsection{3 Índices de sensibilidade para o equilíbrio endêmico}

Para evidenciarmos os parâmetros importantes na prevalência da doença, faremos um estudo do índice de sensibilidade do equilíbrio endêmico. Para isso aplicaremos novamente o método proposto por Chitnis et al., em [CHC08], aos 3 modelos.

Sejam $n$ e $m$, o número de variáveis e o número de parâmetros de cada modelo, respectivamente. Vamos redefinir as variáveis de estado no equilíbrio endêmico $\left(S_{h}, I_{h}, R_{h}, I_{v}, N_{h}\right)$ como $x_{i}$, para $i=1, \cdots, n$, e os parâmetros, vamos redefini-los como $p_{j}$, para $j=1, \cdots, m$.

A equação para cada modelo avaliada no equilíbrio endêmico é dada por:

$$
h_{i}\left(x_{i}, p_{j}\right)=0
$$

para $i=1, \cdots, n$ e $j=1, \cdots, m$.

Avaliando as derivadas parciais da equação (3.3) para $1 \leq i \leq n$ e $1 \leq j \leq m$, obteremos um sistema da seguinte forma:

$$
\frac{d h_{k}}{d p_{j}}=\sum_{i=1}^{n}\left(\frac{\partial h_{k}}{\partial x_{i}} \frac{\partial x_{i}}{\partial p_{j}}\right)+\sum_{l=1}^{m}\left(\frac{\partial h_{k}}{\partial p_{l}} \frac{\partial p_{l}}{\partial p_{j}}\right)=0
$$

para $1 \leq k \leq n$ e $1 \leq j \leq m$.

Entretanto, $\frac{\partial p_{l}}{\partial p_{j}}=0$ se $l \neq j$ então, a equação (3.4) reduz-se à:

$$
\sum_{i=1}^{n}\left(\frac{\partial h_{k}}{\partial x_{i}} \frac{\partial x_{i}}{\partial p_{j}}\right)=-\frac{\partial h_{k}}{\partial p_{j}} .
$$

Estas equações são desacopladas com respeito aos parâmetros $p_{j}, j=1, \cdots, m$, por outro lado, são acopladas em termos das funções $h_{k}, k=1, \cdots, n$. Portanto a equação (3.5) forma um sistema linear com $n$ equações acopladas que podem ser escritas da forma matricial:

$$
A z^{(j)}=b^{(j)}
$$


onde $A$ é uma matriz de dimensão $(n \times n)$ e corresponde a matriz jacobiana do modelo, $z^{(j)}$ é o vetor desconhecido de dimensão $(n \times 1)$ e $b^{(j)}$ é o vetor de dimensão $(n \times 1)$.

A matriz $A$ é conhecida desde que o valor dos parâmetros para cada modelo é conhecido e estão descritos na Tabela 3.2. Os valores dos equilíbrios endêmicos foram calculados e são apresentados na Tabela 3.1. Da mesma forma, é possível avaliar o vetor $b^{(j)}$ calculando a derivada de (3.3) com respeito aos parâmetros de cada modelo. Observe que a matriz $A$ será sempre a mesma, entretanto, o vetor $b^{(j)}$ mudará conforme cada avaliação de parâmetro.

Resolvendo o sistema (3.6) para $z^{(j)}$, vamos obter $\frac{\partial x_{i}}{\partial p_{j}}$, para $1 \leq i \leq n$ e $1 \leq j \leq m$. Por fim, multiplicando $\frac{\partial x_{i}}{\partial p_{j}}$ por $\frac{p_{j}}{x_{i}}$, conforme a Definição 3.4.1, vamos obter o índice de sensibilidade dos modelos para cada variável de estado no equilíbrio endêmico, $x_{i}$, com relação aos parâmetros, $p_{j}$.

Aplicando a metodologia descrita para os 3 modelos, obtivemos os resultados descritos nas Tabelas 3.5, 3.6 e 3.7 .

\begin{tabular}{c|c|c|c}
\hline & $S_{h}^{*}$ & $I_{h}^{*}$ & $I_{v}^{*}$ \\
\hline$\mu_{h}$ & +0.0018 & $+\mathbf{0 . 9 9}$ & +1.0 \\
$b$ & $\mathbf{- 3 . 6 7}$ & +0.27 & $+\mathbf{2 . 9 5}$ \\
$\beta_{h}$ & -0.99 & +0.076 & +0.076 \\
$A$ & -0.99 & +0.076 & +0.076 \\
$\mu_{v}$ & $+\mathbf{1 . 9 9}$ & -0.15 & $\mathbf{- 1 . 1 5 0 9}$ \\
$m$ & 0 & 0 & 0 \\
$\beta_{v}$ & -1.0 & +0.076 & +1.0769 \\
$\gamma_{h}$ & +1.0 & $\mathbf{- 1 . 0 7 4 5}$ & -1.076 \\
\hline
\end{tabular}

Tabela 3.5: Índices de sensibilidade, $\Gamma_{p_{j}}^{x_{i}}=\frac{\partial x_{i}}{\partial p_{j}} \frac{p_{j}}{x_{i}}$, das varíaveis do modelo 1 no equilibrio endêmico, com relação aos parâmetros descritos na Tabela 3.2.

Com relação ao modelo 1 , a taxa de picadas do vetor, $b$, continua sendo o parâmetro que apresenta maior índice de sensibilidade. O aumento (redução) em $1 \%$ da taxa de picadas do vetor ocasionará um decréscimo (acréscimo) de 3,67\% na proporção de suscetíveis. Para a proporção de humanos infectados, o parâmetro que apresenta maior variabilidade é a taxa de recuperação de humanos, $\gamma_{h}$. Um aumento (diminuição) de 1\% neste parâmetro acarretará num decréscimo (acréscimo) de 1,074\% na proporção de humanos infectados. Na proporção de vetores infectados, a taxa de picadas, $b$, apresenta o maior índice de sensibilidade. Aumentando (diminuindo) este parâmetro em 1\% teremos um decréscimo (acréscimo) de $1,15 \%$ na proporção de vetores infectados. O crescimento do parâmetro demográfico, $\mu_{h}$, apresenta maior sensibilidade com relação a proporção de humanos infectados.

As Figuras 3.6 e 3.7 verificam os índices de sensibilidade do equilíbrio endêmico, obtidos para o modelo 1 , com relação a taxa de picadas do vetor e a taxa de morte do vetor.

Da Figura 3.6 podemos observar que uma pequena variação de $1 \%$ no parâmetro $b$ apresenta impacto nas soluções do modelo 1. Conforme diminuímos o valor deste parâmetro, temos um aumento na proporção de suscetíveis e uma diminuição nas proporções de humanos e vetores infectados. Por outro lado, ao mesmo tempo em que a dinâmica é mais rápida quando este parâmetro é aumentado, temos um decréscimo na proporção de humanos suscetíveis e um acréscimo nas proporções de humanos e vetores infectados. 


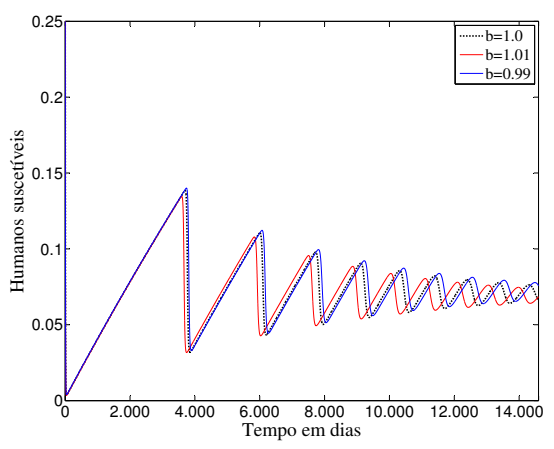

(a) Variação de humanos suscetíveis com relação a taxa de picadas do vetor

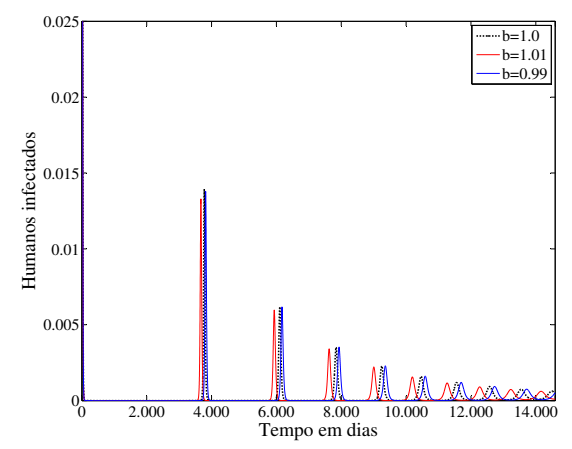

(b) Variação de humanos infectados com relação a taxa de picadas do vetor

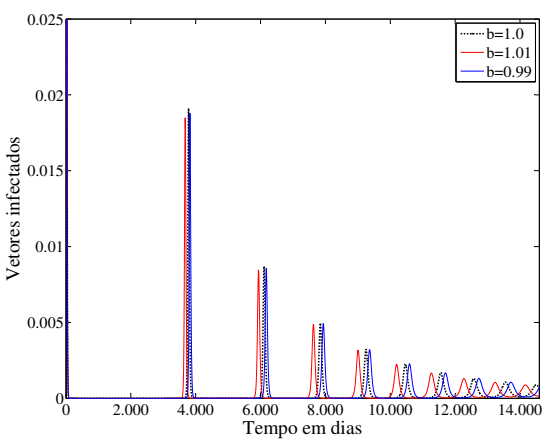

(c) Variação de vetores infectados com relação a taxa de picadas do vetor

Figura 3.6: Variações nas soluções do modelo 1 com relação a variação de $1 \%$ na taxa de picadas do vetor. A linha tracejada corresponde a solução do modelo para o valor do parâmetro b, conforme definido na Tabela 3.2.

Variações na taxa de morte do vetor também apresentam algumas mudanças nas soluções do modelo 1. De acordo com a Figura 3.7, conforme aumentamos a taxa de morte do vetor teremos um impacto maior na proporção de vetores infectados. 


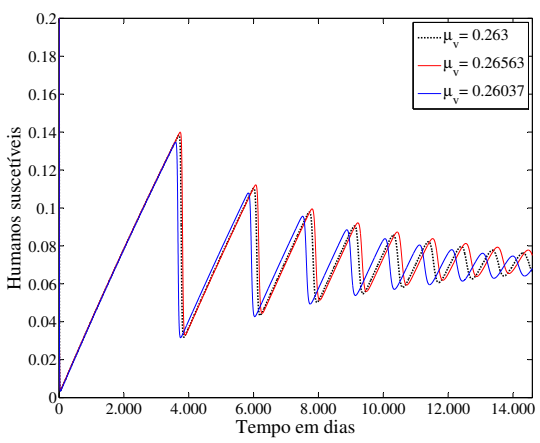

(a) Variação de humanos suscetíveis com relação a taxa de morte do vetor

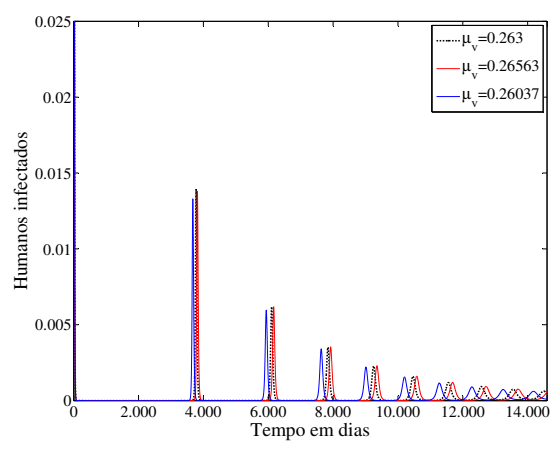

(b) Variação de humanos infectados com relação a taxa de morte do vetor

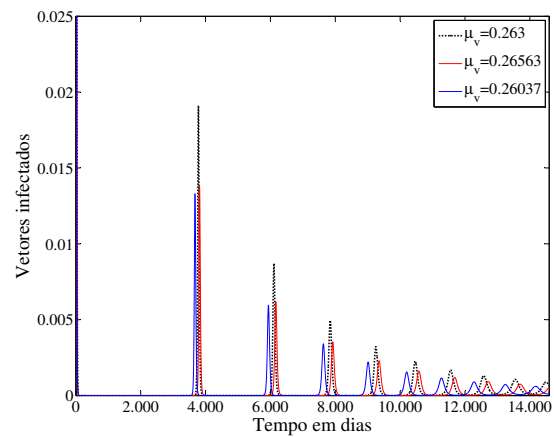

(c) Variação de vetores infectados com relação a taxa de morte do vetor

Figura 3.7: Variações nas soluções do modelo 1 com relação a variação de $1 \%$ na taxa de morte do vetor. A linha tracejada corresponde a solução do modelo para o valor do parâmetro $\mu_{v}$, conforme definido na Tabela 3.2.

A Tabela 3.6, apresenta os índices de sensibilidade do equilíbrio endêmico referentes aos parâmetros do modelo 2 .

\begin{tabular}{c|c|c|c}
\hline & $S_{h}^{*}$ & $I_{h}^{*}$ & $I_{v}^{*}$ \\
\hline$\nu_{h}$ & +0.0279 & $+\mathbf{0 . 9 9}$ & +0.97 \\
$\lambda_{h}$ & $\mathbf{- 0 . 9 9 7}$ & +0.16 & +0.15 \\
$\alpha_{h}$ & +0.0066 & -0.0069 & -0.0067 \\
$\gamma_{h}$ & +0.955 & $\mathbf{- 1 . 1 3}$ & -1.11 \\
$\lambda_{v}$ & -0.97 & +0.16 & $+\mathbf{1 . 1 3}$ \\
$\mu_{v}$ & $+\mathbf{0 . 9 8}$ & -0.16 & $\mathbf{- 1 . 1 4}$ \\
\hline
\end{tabular}

Tabela 3.6: Índices de sensibilidade, $\Gamma_{p_{j}}^{x_{i}}=\frac{\partial x_{i}}{\partial p_{j}} \frac{p_{j}}{x_{i}}$, das varíaveis do modelo 2 no equilibrio endêmico, com relação aos parâmetros descritos na Tabela 3.2.

Para o modelo 2 os parâmetros mais sensíveis são similares ao que foi evidenciado para o modelo 1 . A taxa de morte do vetor, $\mu_{v}$, a taxa de recuperação de humanos, $\gamma_{h}$ e a probabilidade de transmissão de humano para vetor, $\lambda_{v}$, correspondem as maiores variações.

Abaixo na Figura 3.8, apresentamos os resultados obtidos pela análise de sensibilidade do equilíbrio endêmico com relação a taxa de recuperação de humanos, $\gamma_{h}$. Novamente, temos que o aumento na taxa de humanos recuperados apresenta maior impacto na redução de humanos infectados e esse efeito por sua vez também diminui o número de vetores infectados. 


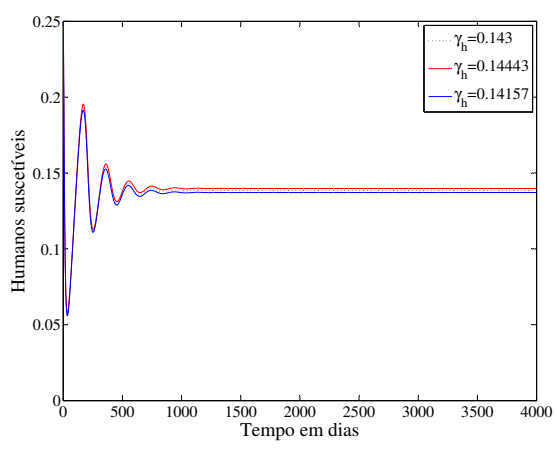

(a) Variação de humanos suscetíveis com relação a taxa de recuperação de humanos

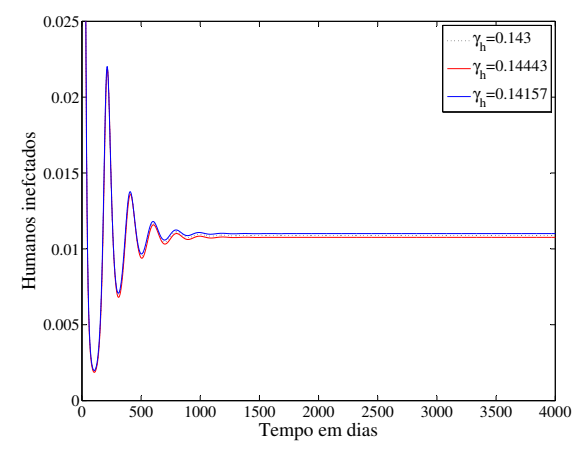

(b) Variação de humanos infectados com relação a taxa de recuperação de humanos

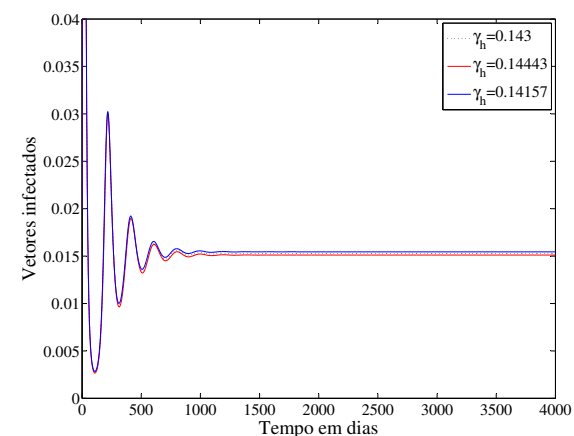

(c) Variação de vetores infectados com relação a taxa de recuperação de humanos

Figura 3.8: Variações nas soluções do modelo 2 com relação a variação na taxa de recuperação de humanos. A linha tracejada corresponde a solução do modelo para o valor do parâmetro $\gamma_{h}$, conforme definido na Tabela 3.2.

A probabilidade de transmissão de humano para vetor apresenta maior sensibilidade a proporção de vetores infectados, uma vez que a cada novo contato entre humanos infectado e vetores suscetíveis temos um aumento de vetores infectados. Esses resultados são evidenciados pela Figura 3.9. 


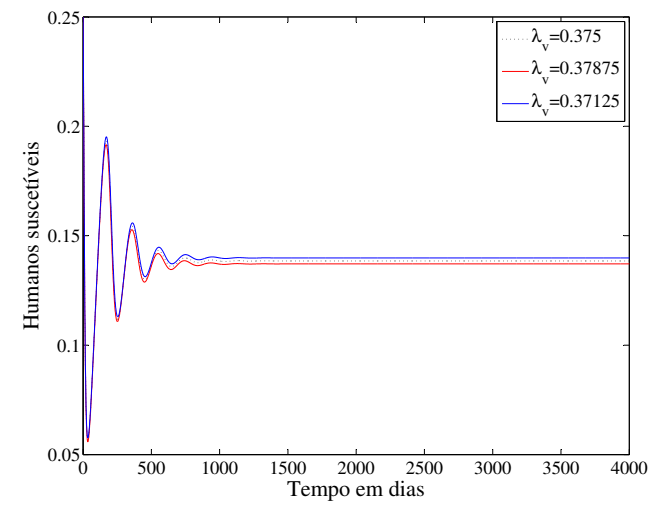

(a) Variação de humanos suscetíveis com relação a taxa de contato efetivo de humano para vetor

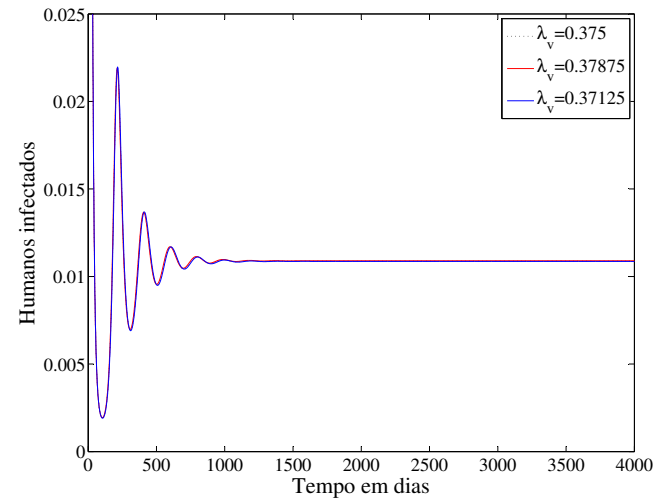

(b) Variação de humanos infectados com relação a taxa de contato efetivo de humano para vetor

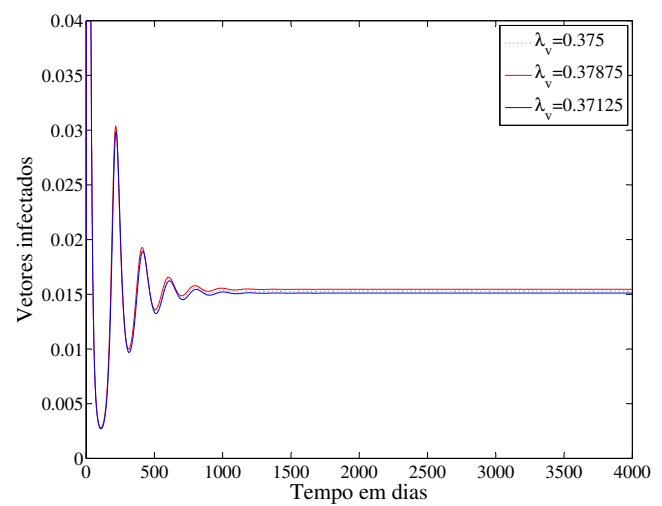

(c) Variação de vetores infectados com relação a taxa de contato efetivo de humano para vetor

Figura 3.9: Variações nas soluções do modelo 2 com relação a variação na taxa de contato efetivo de humano para vetor. A linha tracejada corresponde a solução do modelo para o valor do parâmetro $\lambda_{v}$, conforme definido na Tabela 3.2.

Finalmente, apresentaremos os índices de sensibilidade do equilíbrio endêmico, referentes aos parâmetros do modelo 3, através da Tabela 3.7.

\begin{tabular}{c|c|c|c|c|c}
\hline & $S_{h}^{*}$ & $I_{h}^{*}$ & $R_{h}^{*}$ & $I_{v}^{*}$ & $N_{h}^{*}$ \\
\hline$\lambda$ & 0 & 0 & 0 & 0 & 0 \\
$\mu_{h}$ & +0.00028 & $+\mathbf{1 . 0}$ & -0.00014 & $+\mathbf{1 . 0}$ & 0 \\
$k$ & +0.99 & -0.16 & -0.16 & -0.16 & +1.0 \\
$D$ & -0.99 & +0.16 & +0.16 & +0.16 & 0 \\
$\mu_{v}$ & $+\mathbf{1 . 9 9}$ & -0.33 & -0.33 & $\mathbf{- 1 . 3 3}$ & 0 \\
$b$ & $\mathbf{- 2 . 0}$ & +0.33 & +0.33 & $+\mathbf{1 . 3 3}$ & 0 \\
$\beta_{h}$ & -0.99 & +0.16 & +0.16 & +0.16 & 0 \\
$r$ & +1.0 & $\mathbf{- 1 . 1 6}$ & -0.16 & -1.17 & 0 \\
$\beta_{v}$ & -1.0 & +0.16 & +0.16 & +1.17 & 0 \\
\hline
\end{tabular}

Tabela 3.7: Índices de sensibilidade, $\Gamma_{p_{j}}^{x_{i}}=\frac{\partial x_{i}}{\partial p_{j}} \frac{p_{j}}{x_{i}}$, das varíaveis do modelo 3 no equilibrio endêmico, com relação aos parâmetros descritos na Tabela 3.2. 


\begin{tabular}{c|c|c|c|c|c}
\hline & \multicolumn{5}{|c}{ Variaçoses no equilíbrio } \\
& $S_{h}^{*}$ & $I_{h}^{*}$ & $R_{h}^{*}$ & $I_{v}^{*}$ & $N_{h}^{*}$ \\
\hline$\lambda$ & $0 \%$ & $0 \%$ & $0 \%$ & $0 \%$ & $0 \%$ \\
\hline$\mu_{h}$ & $+0.00028 \%$ & $+1 \%$ & $-0.00014 \%$ & $+1 \%$ & $0 \%$ \\
\hline$k$ & $+0.99 \%$ & $-0.16 \%$ & $-0.16 \%$ & $-0.16 \%$ & $+1 \%$ \\
\hline$D$ & $-0.99 \%$ & $+0.16 \%$ & $+0.16 \%$ & $+0.16 \%$ & $0 \%$ \\
\hline$\mu_{v}$ & $+1.99 \%$ & $-0.33 \%$ & $-0.33 \%$ & $-1.33 \%$ & $0 \%$ \\
\hline$b$ & $-2 \%$ & $+0.33 \%$ & $+0.33 \%$ & $+1.33 \%$ & $0 \%$ \\
\hline$\beta_{h}$ & $-0.99 \%$ & $+0.16 \%$ & $+0.16 \%$ & $+0.16 \%$ & $0 \%$ \\
\hline$r$ & $+1 \%$ & $-1.16 \%$ & $-0.16 \%$ & $-1.17 \%$ & $0 \%$ \\
\hline$\beta_{v}$ & $-1 \%$ & $+0.16 \%$ & $+0.16 \%$ & $+1.17 \%$ & $0 \%$ \\
\hline
\end{tabular}

Tabela 3.8: Variações nas proporções do equilıbrio endêmico considerando uma variação de $1 \%$ nos parâmetros do modelo 3.

infectados apresentam maior sensibilidade com relação a taxa de recuperação de humanos, $r$, e a taxa de morte de humanos, $\mu_{h}$. Variando o parâmetro $\mu_{h}$ em $1 \%$ vamos ter um aumento de $1 \%$ na proporção de humanos infetados enquanto que, um aumento de $1 \%$ no parâmetro $r$, corresponde a uma redução de $1,16 \%$ na proporção de humanos infectados.

Como a convergência das soluções deste modelo é mais lenta, apresentaremos uma tabela com a variação de alguns parâmetros identificados pela Tabela 3.7 e o seu impacto no valor das proporções no equilíbrio endêmico. Considerando as proporções no equilíbrio $S_{h}^{*}=0.1407, I_{h}^{*}=0.00009, R_{h}^{*}=0.8592, I_{v}^{*}=0.000128$ e $N_{h}^{*}=20000$ obtidas para a base de valores da Tabela 3.2 , obtivemos as seguintes variações na prevalência da doença com relação a variações na taxa de nascimento de humanos, taxa de morte de humanos, taxa de picadas do vetor e taxa de recuperação de humanos.

Os resultados da análise de sensibilidade são coerentes com a tabela acima. A taxa de picadas do vetor por apresentar maior índice de sensibilidade e corresponde ao parâmetro que mais impacta na prevalência da doença. A taxa de morte do vetor, $\mu_{v}$, aparece com o segundo maior índice de sensibilidade. O parâmetro $r$ também é responsável por um impacto considerável na proporção de infectados. O aumento na taxa de recuperação de humanos representa um aumento na proporção de humanos suscetíveis e por outro lado, representa uma diminuição na proporção de infectados e humanos recuperados.

Portanto, esta análise sugere estratégias no controle da propagação da dengue. Enquanto o desenvolvimento de uma vacina segura e tetravalente para a dengue ainda está em processo, o controle do vetor é ainda a única estratégia viável para minimizar o número de casos da doença. Nosso estudo indica que parâmetros relacionados a dinâmica de transmissão e dinâmica do vetor, tais como a taxa de picadas do vetor e a taxa de morte do vetor são estratégias mais eficientes para reduzir a propagação inicial e prevalência da doença. Uma alternativa para reduzirmos a taxa de picadas do vetor é o uso de repelentes. Como o mosquito da dengue possui hábitos diurnos o uso de repelentes é uma estratégia viável para o controle da taxa de picadas do vetor.

Outra estratégia que pode ser efetiva no controle da taxa de morte do vetor é a pulverização com inseticidadas, como o fumacê. Atualmente esta técnica está sendo empregada, porém sem muito sucesso. Isso ocorre, pois o efeito do inseticida tem ação temporária e pontual. Outro parâmetro que destacou-se desta análise foi a taxa de recuperação de humanos. Este parâmetro pode ser minimizado com um rápido diagnóstico e tratamento de indivíduos infecciosos. Para dengue em particular, o controle deste parâmetro pode não ser uma alternativa eficiente por dois fatores, um deles está relacionado ao diagnóstico da doença. Em geral, cerca de 1\% das notificações de dengue são confirmadas por exame sorológico. Outro fator relevante corresponde ao aumento no número de casos assintomáticos.

Através deste estudo mapeamos os parâmetros determinantes para a prevalência da dengue e com isso foi possível identificar as falhas no controle dessa doença. Embora as estratégias de controle tenham sido 
avaliadas isoladamente estas estratégias devem ser mais eficientes se aplicadas combinadas. A partir desta proposta surgem outras indagações, como por exemplo o estudo da viabilidade econômica da implementação de estratégias de controle do vetor bem como a inclusão de um compartimento para indivíduos assintomáticos e a sua contribuição para o aumento no número de indivíduos infectados. 


\section{Conclusões}

Através do surgimento das doenças infecciosas, observou-se a evolução da epidemiologia matemática. Os primeiros conceitos em epidemiologia matemática sugiram a partir da necessidade humana em entender quais eram os mecanismos de propagação dessas doenças. Entretanto, a teoria que embasa a epidemiologia matemática, não surgiu essencialmente a partir de interesses matemáticos e sim, como uma ferramenta em saúde pública. Atualmente, com o aprimoramento das técnicas em medicina e a agregação dessas informações aos modelos de propagação de doenças, a matemática enquanto teoria vem sendo explorada e aplicada no estudo de epidemias.

Neste trabalho avaliamos o comportamento qualitativo das soluções de um modelo determinístico de propagação de dengue do tipo SIR com acoplamento da dinâmica do vetor. Para o estudo foram consideradas três hipóteses sobre o tamanho total da população humana descritas pelos modelos 1,2 e 3 . As hipóteses do modelo base são as mesmas exceto pelo fato que no modelo 2, cujo tamanho da população humana é governado por uma função de crescimento exponencial, o sistema admite uma taxa de morte causada pela doença.

O comportamento das soluções do modelo matemático foi avaliado em termos das proporções das populações de humanos e vetores. Para cada um dos modelos foi desenvolvida a mesma análise. Inicialmente definimos condições de existência e unicidade das soluções dos modelos assim como, definimos o domínio epidemiológico destas soluções. Garantidas as condições de existência e unicidade das soluções foi calculada a expressão para o número de reprodutibilidade basal, $R_{o}$.

O parâmetro $R_{o}$ apresentou dependência exclusiva dos parâmetros dos modelos e da variável $N_{h}$. As expressões do $R_{o}$ para os modelos 1 e 3 foram basicamente as mesmas. Entretanto, para o modelo 3, a relação inversa entre este parâmetro e a variável $N_{h}$ faz com que esta quantidade diminua com o passar do tempo. No caso do modelo 2, a expressão para o $R_{o}$ apresenta dependência somente dos parâmetros e portanto este valor é fixo.

Supondo que não sejam desenvolvidas estratégias de controle da doença, de acordo com as estimativas fornecidas pelo modelo 3, vamos ter ao longo do tempo uma diminuição no potencial de propragação inicial da doença devido ao decaimento do parâmetro $R_{o}$. Entretanto, se considerarmos que o parâmetro $R_{o}$ é calculado no início da epidemia, as estimativas fornecidas pelos modelos 1 e 3 são basicamente as mesmas.

A estimativa fornecida para o modelo 2 teoricamente seria melhor, pois é uma das menores estimativas. Entretanto, apesar de considerar um crescimento exponencial para a população humana, o valor de $R_{o}$ não apresenta dependência com relação a esta variável. Além disso, considerando que a dengue é uma doença com baixa letalidade e de acordo com os dados demográficos disponíveis, a hipótese sobre considerar uma função de crescimento exponencial para a população humana parece pouco razoável.

Apesar dos modelos 1 e 3 apresentarem estimativas semelhantes com relação a propagação inicial da doença vemos que as hipóteses sobre o tamanho da população humana afetam de forma mais significativa as proporções do equilíbrio endêmico, ou seja, afetam a prevalência da doença. De fato, nosso estudo teórico demonstrou que as hipóteses sobre o tamanho da população podem afetar o comportamento qualitativo das soluções do modelo matemático assim como parâmetros relativos a dinâmica do vetor.

A partir do cálculo do $R_{o}$ foi possível obter os pontos de equilíbrio dos modelos. De acordo com as hipóteses e estrutura dos modelos matemáticos foram obtidos dois pontos de equilíbrio, definidos epidemio- 
logicamente como equilíbrio livre de doença e equilíbrio endêmico. Para os três modelos foram apresentadas condições que garantem a estabilidade global do equilíbrio livre de doença se $R_{o} \leq 1$. Mostramos também que para $R_{o}>1$ o equilíbrio endêmico é globamente estável.

Verificadas as condições de estabilidade dos equilíbrios nosso próximo passo foi analisar os modelos de propagação de dengue avaliando o índice de sensibilidade do número de reprodução basal, $R_{o}$, e do equilíbrio endêmico, $E_{2}$. Desde que, o parâmetro $R_{o}$ mede a transmissão inicial da doença e o equilíbrio endêmico é uma medida de prevalência da doença, a análise de sensibilidade permitiu-nos determinar a importância relativa de diferentes parâmetros na transmissão e prevalência da doença. A escolha do valor dos parâmetros foi obtida da literatura respeitando-se as hipóteses de construção dos modelos.

De maneira geral, para os três modelos o parâmetro mais sensível para a transmissão inicial da doença é a taxa de picadas do vetor. Outros parâmetros importantes correspondem a taxa de morte do vetor, a taxa de transmisão de humanos e vetores, a taxa de transmissão de vetores e humanos, a taxa de recrutamento de vetores e a taxa de recuperação de humanos. Estratégias que reduzem o contato entre humanos e vetores pode ser uma alternativa de controle para a taxa de picadas. $\mathrm{O}$ uso de repelentes e telas de proteção podem ser uma alternativa eficiente para a redução da transmissão inicial da doença.

Com relação a análise de sensibilidade obtida para o equilíbrio endêmico, nosso estudo evidenciou que parâmetros relacionados a dinâmica de transmissão entre humanos e vetores, bem como parâmetros relacionados a dinâmica do vetor apresentam índices de sensibilidade consideráveis para os três modelos. Em particular, para a proporção de humanos infectados nossa análise demonstrou que estratégias de intervenção que afetem a taxa de recuperação de humanos devem ser mais efetivas na redução das frações de infectados. Por outro lado, a taxa de picadas do vetor e a taxa de mortalidade do vetor também apresentam índices de sensibilidade consideráveis na prevalência da doença.

Os valores particulares para os índices de sensibilidade do equilíbrio endêmico e do $R_{o}$ para diferentes parâmetros dependem dos valores escolhidos para estes parâmetros e das hipóteses sob as quais os modelos são estrututrados. Portanto, esta análise além de nos indicar possíveis estratégias de controle sinaliza quais parâmetros devem ser medidos ou inferidos com uma maior precisão.

A partir do mapeamento dos modelos de propagação desenvolvido pelo estudo teórico e pela análise de sensibilidade, uma possível continuidade deste trabalho consiste em propor uma comparação entre diferentes estratégias de controle avaliando-se a viabilidade econômica de implementação destas estratégias. O efeito da sazonalidade também pode ser incluído para verificarmos o impacto no número, na expectativa de vida e na taxa de picadas do vetor. 


\section{Apêndice A}

\section{Definições Preliminares}

\section{A.1 Noções de Estabilidade}

Seja $D \in \mathbb{R}^{n}$ um conjunto aberto e seja $x \longmapsto F(x) \in \mathbb{R}^{n}$ uma função de classe $C^{1}$ definida em D. Considere o sistema autônomo em $\mathbb{R}^{n}$ dado por:

$$
x^{\prime}=F(x) .
$$

Definição A.1.1. Denotaremos a solução de (A.1) satisfazendo $x(0)=x_{o}$ como $\phi\left(t, x_{o}\right)$.

Definição A.1.2. Seja $I(x)$ o intervalo máximo de definição da solução $\phi\left(t, x_{o}\right)$ definido como $t_{+}(x)=\sup t: t \in I(x)$ e $t_{-}(x)=\sup t: t \in I(x)$.

Definição A.1.3. A órbita de um ponto $x \in D$ corresponde ao conjunto $\gamma(x)=\{\phi(t, x): t \in I(x)\}$. A semi-órbita positiva de $x$ é o conjunto $\gamma^{+}(x)=\{\phi(t, x): t \in I(x), t>0\}$. De maneira análoga, $\gamma^{-}(x)=$ $\{\phi(t, x): t \in I(x), t<0\}$ corresponde a semi-órbita negativa de $x$.

Definição A.1.4. Denota-se por:

$$
\omega(x)=\left\{y \in D: \exists\left\{t_{n}\right\}, t_{n} \rightarrow t_{+}(x) \quad e \quad \phi\left(t_{n}, x\right) \rightarrow y\right\},
$$

o conjunto $\omega$-limite de $x$.

Definição A.1.5. Denota-se por:

$$
\alpha(x)=\left\{y \in D: \exists\left\{t_{n}\right\}, t_{n} \rightarrow t_{-}(x) \quad e \quad \phi\left(t_{n}, x\right) \rightarrow y\right\},
$$

o conjunto $\alpha$-limite de $x$.

Definição A.1.6. Uma órbita fechada $\gamma$, com periodo $T>0$ é imagem de uma solução $\phi(., x): \mathbb{R} \rightarrow D$, tal que, $\phi(t+T, x)=\phi(t, x)$ para todo $t$.

Definição A.1.7. Um ponto $x^{*}$ é um ponto de equilıbrio para o sistema (A.1) se, $F\left(x^{*}\right)=0$.

Definição A.1.8. Dizemos que $x^{*}$ é um ponto de equilíbrio estável se, para qualquer vizinhança $U \subset \mathbb{R}^{n}$ de $x^{*}$, existe uma vizinhança $V \subset \mathbb{R}^{n}$ de $x^{*}$, tal que $V \subset U$ e $\phi_{t}(x) \in U$, para quaisquer $x \in V, t>0$.

Definição A.1.9. Dizemos que $x^{*}$ é um ponto de equilíbrio assintoticamente estável se, para qualquer vizinhança $U \subset \mathbb{R}^{n}$ de $x^{*}$, existe uma vizinhança $V \subset \mathbb{R}^{n}$ de $x^{*}$, tal que $V \subset U$ e $\phi(t, x) \in U$, para quaisquer $x \in V, t>0$ e $\lim _{t \rightarrow \infty} \phi_{t}(x)=x^{*}$ para quaisquer $x \in V, t>0$. 
Dada a definição de ponto de equilíbrio evidenciaremos alguns critérios que são amplamente usados na análise de estabilidade desses equilíbrios. Dentre eles estão o critério de Routh-Hurwitz e o critério de Lyapunov (consultar [Lee08], [HSD12], [Tel79]).

Definição A.1.10. Um polinômio $L(p)$ é dito estável se todas as suas raízes têm parte real negativa.

Teorema A.1.11 (Critério de Routh-Hurwitz para polinômios de grau 3). O polinômio

$$
L(p)=a_{o} p^{3}+a_{1} p^{2}+a_{2} p+a_{3} \quad, a_{o}>0
$$

com coeficientes reais, é estável se, e somente se, $a_{1}, a_{2}, a_{3}$ são positivos e $a_{1} a_{2}>a_{o} a_{3}$.

Definição A.1.12. Seja $L: U \rightarrow \mathbb{R}$ uma função diferenciável, definida um aberto $U \in \mathbb{R}^{n}$ que contém um ponto de equilíbrio $x^{*}$ do sistema (A.1). Se $\phi(t, x)$ é solução de (A.1) passando por $x$ em $t=0$, então

$$
L^{\prime}(x)=\left.\frac{d}{d t}\right|_{t=0} L(\phi(t, x))
$$

Consequentemente, se $L^{\prime}(x)$ é negativa, $L$ decresce ao longo das soluções através de $x$.

Teorema A.1.13 (Estabilidade de Lyapunov). Seja $x^{*}$, um ponto de equilibrio para o sistema (A.1). Seja $L: U \rightarrow \mathbb{R}$ uma função diferenciável, definida um aberto $U \in \mathbb{R}^{\ltimes}$ que contém $x^{*}$. Suponha ainda que:

i. $L\left(x^{*}\right)=0$ e $L(x)>0$ se, $x \neq x^{*} e$

ii. $L^{\prime} \leq 0$ em $U-x^{*}$ então $x^{*}$ é estável. Além disso, se L satisfaz

iii. $L^{\prime}<0$ em $U-x^{*}$ então $x^{*}$ é assintoticamente estável.

A função $L$, satisfazendo as condições $i$. e $i i$. é dita função de Lyapunov para $x^{*}$.

Definição A.1.14. Seja $x^{*}$ um ponto de equilíbrio de (A.1) assintoticamente estável. O conjunto $B\left(x^{*}\right)=$ $\left\{x \in D ; \phi(t, x) \rightarrow x^{*}\right.$ quando $\left.t \rightarrow \infty\right\}$ chama-se bacia de atração ou variedade estável de $x^{*}$.

Definição A.1.15. Um conjunto $\Gamma$ é dito invariante se, para cada $x \in \Gamma, \phi(t, x)$ está definido em $\Gamma$ para todo $t \in \mathbb{R}$. Além disso, o conjunto $\Gamma$ é positivamente invariante se, para cada $x \in \Gamma, \phi(t, x)$ está definido em $\Gamma$ para todo $t \geq 0$.

Definição A.1.16. Um ponto de equilibrio $x^{*}$ do sistema (A.1) diz-se instável quando não for estável.

Para uma revisão mais detalhada destes conceitos indicamos Sotomayor, em [Tel79] e Hirsch et al., em [HSD12].

\section{A.2 Resultados da teoria de sistemas competitivos e estabilidade de órbitas periódicas}

Introduziremos aqui, alguns conceitos e resultados sobre a teoria de sistemas competitivos e estabilidade de órbitas periódicas, que serão utilizados durante o capítulo 2 para garantirmos a estabilidade dos pontos de equilíbrio. Para uma revisão mais detalhada sobre sistemas competitivos indicamos os trabalhos de Hirsch, em [Hir90] e [Hir89], Smith, em [Smi95] e [Smi88] e Butler et al., em [BFW86]. Sobre estabilidade de órbitas periódicas indicamos os trabalhos de Muldowney, em [Mul90] e Smith e Zhu, em [ZS94]. 
Definição A.2.1. O sistema (A.1) é dito cooperativo em D se, para alguma matriz diagonal $H=\operatorname{diag}\left(\epsilon_{1}, \epsilon_{2}, \cdots, \epsilon_{n}\right)$, onde cada $\epsilon_{i}, i=1, n$ é 1 ou -1 e $H(D F(x)) H$ tem elementos não negativos fora da diagonal para $x \in D$ e, $D F(x)$ é a matriz jacobiana associada ao sistema (A.1).

Uma importante característica de sistemas tridimensionais competitivos e cooperativos é que eles possuem a propriedade de Poincaré-Bendixson:

Teorema A.2.2 (Propriedade de Poincaré-Bendixson para sistemas competitivos e cooperativos tridimensionais). Um conjunto $\omega$-limite compacto de um sistema competitivo ou cooperativo em $\mathbb{R}^{3}$ que, não contém pontos de equilíbrio é uma órbita periódica.

Sistemas competitivos e cooperativos, induzem fluxos em $\mathbb{R}^{n}$ com boas propriedades em relação a ordenação parcial, definida como:

Definição A.2.3. Em $\mathbb{R}^{n}$ temos uma relação de ordem. Dizemos que $x<y, x, y \in \mathbb{R}^{n}$ se, $x_{i}<y_{i}$ para todo $i=1, \cdots, n$. Da mesma forma, dizemos que $x \leq y, x, y \in \mathbb{R}^{n}$ se, $x_{i} \leq y_{i}$ para todo $i=1, \cdots, n$.

Proposição A.2.4. Se D é convexo, o fluxo de um sistema competitivo preserva, para $t<0$ a ordenação parcial em $\mathbb{R}^{n}$ definida pelo octante

$$
K=\left(x_{1}, x_{2}, \cdots, x_{n}\right) \in \mathbb{R}^{n}: \epsilon_{i} x_{i} \geq 0, i=1, n .
$$

Prova: Ver Smith [Smi88] .

Observação A.2.5. O conceito de competitividade como definido acima é mais geral do que o conceito definido por Smith, em [Smi88], pois a ordenação parcial não é necessariamente definida pela octante padrão em $\mathbb{R}^{\ltimes}$. Entretanto, através de uma mudança linear de variáveis $y=H x$, um sistema competitivo, conforme a definição (2.1.3), pode ser transformado em um sistema competitivo, no sentido descrito em Hirsch (ver [Hir90]). Se $x^{\prime}=F(x)$ é um sistema competitivo com fluxo $\phi(t, x)$ então, o sistema $x^{\prime}=-F(x)$ é um sistema cooperativo com fluxo $\psi(t, x)$, onde $\psi(t, x)=\phi(-t, x)$ e o contrário também se verifica. Assim, notemos que um sistema cooperativo gera um sistema dinâmico monótono e por conseguinte um sistema competitivo tem a propriedade da monotonicidade, pois seu fluxo no tempo reverso é monótono.

Teorema A.2.6. Seja $\Gamma$ uma órbita periódica não trivial de um sistema competitivo em um conjunto convexo $D \subset \mathbb{R}^{3}$ tal que

$$
\Gamma \subset[\bar{a}, \bar{b}] \subset D
$$

onde, $[\bar{a}, \bar{b}]=\{\bar{a} \leq x \leq \bar{b}\}$ e $\leq$ é a ordem lexicográfica em $\mathbb{R}^{3}$. Então $[\bar{a}, \bar{b}]$ contém um ponto de equilíbrio.

Prova: Ver Hirsch [Hir90].

Introduziremos agora um conceito proposto por Muldowney, em [Mul90], que estabelece uma condição suficiente para a estabilidade orbital assintótica de órbitas periódicas.

Definição A.2.7. O sistema (A.1) tem a propriedade da estabilidade de órbitas periódicas se, e somente se, a órbita de qualquer solução periódica $\gamma(t)$ se existir é orbitalmente assintoticamente estável.

Definição A.2.8. Seja $A=\left(a_{i j}\right)$ uma matriz de dimensão $(n \times n)$ e $1 \leq k \leq n$ um inteiro, a $k$-ésima matriz composta aditiva de A é denominada $A^{[k]}$. Esta é uma matriz $N \times N, N=\left(\begin{array}{l}n \\ k\end{array}\right)$ é definida por:

$$
A^{[k]}=\left.D_{+}(I+h A)^{(k)}\right|_{h=0}
$$

onde, $D_{+}$denota a derivada pelo lado direito com relação a h e I corresponde a matriz identidade. 
Por exemplo, quando $n=2, A^{[2]}=\operatorname{traço} A$. Quando $n=3$,

$$
A^{[2]}=\left[\begin{array}{ccc}
a_{11}+a_{22} & a_{23} & -a_{13} \\
a_{32} & a_{11}+a_{33} & a_{12} \\
-a_{31} & a_{21} & a_{22}+a_{33}
\end{array}\right] .
$$

Lema A.2.9. Se $X(t)$ é uma função $n$-vetorial continuamente diferenciável em $a \leq t \leq b$ então, $D_{+}$existe em $a \leq t \leq b$ e $\left|D_{+}\right| X(t)|| \leq\left|X^{\prime}(t)\right|$.

Prova: Ver Hale, em [Hal80], p.30.

Corolário A.2.10 (Desigualdade de Gronwall). Se $\alpha$ é uma constante real, $\psi(t) \geq 0$ e $\phi(t)$ são funções reais continuas para $a \leq t \leq b$ que satisfazem

$$
\phi(t) \leq \alpha+\int_{a}^{t} \psi(s) \phi(s) d s \quad, \quad a \leq t \leq b
$$

então

$$
\phi(t) \leq\left(\exp \int_{a}^{t} \psi(s) \phi(s) d s\right) \alpha \quad, \quad a \leq t \leq b .
$$

Prova: Ver Hale, p.36 [Hal80]. 


\section{Referências Bibliográficas}

[AK09] Ben Adams e Durrell D. Kapan. Man bites mosquito: understanding the contribution of human movement to vector-borne disease dynamics. PLoS ONE, 2009. 5

[AM82] Joan L Aron e Robert M. May. The population dynamics of malaria. Em The population dynamics of infectious diseases: theory and applications, páginas 139-179. Springer, 1982. 17

[AM91] Roy M. Anderson e Robert M. May. Infectious diseases of humans: dynamics and control, volume 28. Oxford: Osford university press, 1991. 4, 7, 17

[And91] Roy M. Anderson. Discussion: the kermack-mckendrick epidemic threshold theorem. Bulletin of mathematical biology, 53(1-2):1-32, 1991. 4

[Ari09] Julien Arino. Diseases in metapopulations. Modeling and dynamics of infectious diseases, $11: 65-123,2009.5$

$\left[\mathrm{B}^{+} 75\right]$ Norman T.J. Bailey et al. The mathematical theory of infectious diseases and its applications. Charles Griffin \& Company Ltd, 5a Crendon Street, High Wycombe, Bucks HP13 6LE., 1975. xviii, 3,4

$\left[\mathrm{B}^{+} 77\right]$ Norman T.J. Bailey et al. Mathematics, statistics, and systems for health. John Wiley and Sons Ltd., Baffins Lane, Chichester, Sussex, 1977. xvii, xviii, 1, 2

[BD94] Sally M. Blower e Hadi Dowlatabadi. Sensitivity and uncertainty analysis of complex models of disease transmission: an hiv model, as an example. International Statistical Review/Revue Internationale de Statistique, páginas 229-243, 1994. 5

[BDG09] Dirk Brockmann, Vincent David e Alejandro Morales Gallardo. Human mobility and spatial disease dynamics. Reviews of nonlinear dynamics and complexity, 2:1-24, 2009. 5

[BFW86] Geoffrey Butler, H.I. Freedman e Paul Waltman. Uniformly persistent systems. Proceedings of the American Mathematical Society, páginas 425-430, 1986. 24, 33, 76

[Bra04] Fred Brauer. Backward bifurcations in simple vaccination models. Journal of Mathematical Analysis and Applications, 298(2):418-431, 2004. 46

[Car12] Jack Carr. Applications of centre manifold theory, volume 35. Springer Science \& Business Media, 2012. 46

[CBLM06] Francisco A.B. Coutinho, Marcelo N. Burattini, Luiz F. Lopez e Eduardo Massad. Threshold conditions for a non-autonomous epidemic system describing the population dynamics of dengue. Bulletin of mathematical biology, 68(8):2263-2282, 2006. 58

[CCS02] Carlos Castillo-Chavez e Baojun Song. An overview of dynamical models of tuberculosis. Summer School on Mathematical Biology, A. Margheri, C. Rebelo, and F. Zanolin, eds., Centro Intemacional de Matematica, Lisbon, accepted, 2002. 4

[CCS04] Carlos Castillo-Chavez e Baojun Song. Dynamical models of tuberculosis and their applications. Mathematical biosciences and engineering, 1(2):361-404, 2004. 4, 21, 39, 46, 47

[CH52] C.F. Curtiss e Joseph O. Hirschfelder. Integration of stiff equations. Proceedings of the National Academy of Sciences of the United States of America, 38(3):235, 1952. 57 
[CHC08] Nakul Chitnis, James M. Hyman e Jim M. Cushing. Determining important parameters in the spread of malaria through the sensitivity analysis of a mathematical model. Bulletin of mathematical biology, 70(5):1272-1296, 2008. xix, 62, 64

[CLL13] Li-Ming Cai, Xue-Zhi Li e Zhaoqiang Li. Dynamical behavior of an epidemic model for a vector-borne disease with direct transmission. Chaos, Solitons \& Fractals, 46:54-64, 2013. 5

[CS78] Vincenzo Capasso e Gabriella Serio. A generalization of the kermack-mckendrick deterministic epidemic model. Mathematical Biosciences, 42(1):43-61, 1978. 4

[dGeEI] Instituto Brasileiro de Geografia e Estatística (IBGE). Censo demográfico 2010. http://www. ibge.gov.br/home/estatistica/populacao/censo2010/default.shtm. Acessado: 30-05-2016. 58

[DH02] Klaus Dietz e J.A.P. Heesterbeek. Daniel bernoulli's epidemiological model revisited. Mathematical biosciences, 180(1):1-21, 2002. 3

[DHCC98] Jonathan Dushoff, Wenzhang Huang e Carlos Castillo-Chavez. Backwards bifurcations and catastrophe in simple models of fatal diseases. Journal of mathematical biology, 36(3):227-248, 1998. 46

[DHM90] Odo Diekmann, J.A.P. Heesterbeek e Johan A.J. Metz. On the definition and the computation of the basic reproduction ratio r 0 in models for infectious diseases in heterogeneous populations. Journal of mathematical biology, 28(4):365-382, 1990. 7, 9, 10, 11, 17

[DHR09] Odo Diekmann, J.A.P. Heesterbeek e M.G. Roberts. The construction of next-generation matrices for compartmental epidemic models. Journal of the Royal Society Interface, página rsif20090386, 2009. $7,11,17,30$

[DPE13] Benjamin D. Dalziel, Babak Pourbohloul e Stephen P. Ellner. Human mobility patterns predict divergent epidemic dynamics among cities. Proceedings of the Royal Society of London B: Biological Sciences, 280(1766):20130763, 2013. 5

[dS] Ministério da Saúde. Boletim epidemiológico. http://portalsaude.saude.gov.br/index.php/ situacao-epidemiologica-dados-dengue. Acessado: 30-05-2016. xviii

[dS10] Daniel Rodrigues da Silva. Um modelo matemático para avaliação do impacto da temperatura na evolução da virulência. Tese de Doutorado, Universidade de São Paulo, 2010. 5

[Dye14] Christopher Dye. After 2015: infectious diseases in a new era of health and development. Phil. Trans. R. Soc. B, 369(1645):20130426, 2014. xiii, 2, 3

[EV98] Lourdes Esteva e Cristobal Vargas. Analysis of a dengue disease transmission model. Mathematical biosciences, 150(2):131-151, 1998. xviii, 13, 14, 58

[EV99] Lourdes Esteva e Cristobal Vargas. A model for dengue disease with variable human population. Journal of mathematical biology, 38(3):220-240, 1999. xviii, 4, 13, 28, 33

[GA85] B.T. Grenfell e Roy M. Anderson. The estimation of age-related rates of infection from case notifications and serological data. Journal of Hygiene, 95(02):419-436, 1985. 6

[GGB08] Salisu Mohammed Garba, Abba B. Gumel e M.R. Abu Bakar. Backward bifurcations in dengue transmission dynamics. Mathematical biosciences, 215(1):11-25, 2008. 39

[GRS96] Walter R. Gilks, Sylvia Richardson e David J. Spiegelhalter. Introducing markov chain monte carlo. Markov chain Monte Carlo in practice, 1:19, 1996. 5

[Hal80] Jack K. Hale. Ordinary Differential Equations, volume 21. Wiley-Interscience, 1980. 78

[HD96] J.A.P. Heesterbeek e K. Dietz. The concept of ro in epidemic theory. Statistica Neerlandica, 50(1):89-110, 1996. 7, 8, 9, 10

[Het76] Herbert W. Hethcote. Qualitative analyses of communicable disease models. Mathematical Biosciences, 28(3):335-356, 1976. xvii 
[Het00] Herbert W. Hethcote. The mathematics of infectious diseases. SIAM review, 42(4):599-653, 2000. xvii, $3,4,7,8,9$

[Hir89] Morris W. Hirsch. Systems of differential equations that are competitive or cooperative. v. convergence in 3-dimensional systems. Journal of differential equations, 80(1):94-106, 1989. 76

[Hir90] Morris W. Hirsch. Systems of differential equations that are competitive or cooperative. iv: Structural stability in three-dimensional systems. SIAM journal on mathematical analysis, $21(5): 1225-1234,1990.22,34,76,77$

[HLVDD89] Herbert W. Hethcote, Mark A. Lewis e Pauline Van Den Driessche. An epidemiological model with a delay and a nonlinear incidence rate. Journal of mathematical biology, 27(1):49-64, 1989. 5

[HNW10] Ernst Hairer, Syvert Paul Nrsett e Gerhard Wanner. Solving Ordinary Differential Equations: Nonstiff problems. v. 2: Stiff and differential-algebraic problems. Springer Verlag, 2010. 58

[HSD12] Morris W. Hirsch, Stephen Smale e Robert L. Devaney. Differential equations, dynamical systems, and an introduction to chaos. Academic press, 2012. 13, 19, 20, 76

[HVdD91] Herbert W. Hethcote e Pauline Van den Driessche. Some epidemiological models with nonlinear incidence. Journal of Mathematical Biology, 29(3):271-287, 1991. 5

[HWSW99] Simon Hales, Phil Weinstein, Yvan Souares e Alistair Woodward. El nino and the dynamics of vectorborne disease transmission. Environmental Health Perspectives, 107(2):99, 1999. 5

[ISS13] Abderrahman Iggidr, Gauthier Sallet e Max O. Souza. Analysis of the dynamics of a class of models for vector-borne diseases with host circulation. Research Report RR-8396, HAL-Inria, Novembro 2013. 5

[KDVH10] Matt J. Keeling, Leon Danon, Matthew C. Vernon e Thomas A. House. Individual identity and movement networks for disease metapopulations. Proceedings of the National Academy of Sciences, 107(19):8866-8870, 2010. 5

[Kee05] Matt Keeling. The implications of network structure for epidemic dynamics. Theoretical population biology, 67(1):1-8, 2005. 5

[KM27] William O. Kermack e Anderson G. McKendrick. A contribution to the mathematical theory of epidemics. Em Proceedings of the Royal Society of London A: Mathematical, Physical and Engineering Sciences, volume 115, páginas 700-721. The Royal Society, 1927. 4

[KM05] Andrei Korobeinikov e Philip K. Maini. Non-linear incidence and stability of infectious disease models. Mathematical Medicine and Biology, 22(2):113-128, 2005. 5

[KR08] Matt J. Keeling e Pejman Rohani. Modeling infectious diseases in humans and animals. Princeton University Press, 2008. 4, 6, 7

[Lee08] Seong Ho Lee. Familias de polinômios estáveis: teoremas de Routh-Hurwitz e Kharitonov. Tese de Doutorado, Universidade de Sao Paulo, 2008. 76

[LHL87] Wei-min Liu, Herbert W. Hethcote e Simon A. Levin. Dynamical behavior of epidemiological models with nonlinear incidence rates. Journal of mathematical biology, 25(4):359-380, 1987. 5

[LJ14] Guihua Li e Zhen Jin. Bifurcation analysis in models for vector-borne diseases with logistic growth. The Scientific World Journal, 2014, 2014. xviii, 13, 39

[LLI86] Wei-min Liu, Simon A. Levin e Yoh Iwasa. Influence of nonlinear incidence rates upon the behavior of sirs epidemiological models. Journal of mathematical biology, 23(2):187-204, 1986. 5

[LM95] Michael Y. Li e James S. Muldowney. Global stability for the seir model in epidemiology. Mathematical biosciences, 125(2):155-164, 1995. xviii, 22, 23, 26, 38 
$\left[\mathrm{LMAM}^{+} 14\right]$ Joseph A. Lewnard, Martial L. Ndeffo Mbah, Jorge A. Alfaro-Murillo, Frederick L. Altice, Luke Bawo, Tolbert G. Nyenswah e Alison P. Galvani. Dynamics and control of ebola virus transmission in montserrado, liberia: a mathematical modelling analysis. The Lancet Infectious Diseases, 14(12):1189-1195, 2014. 4

[MF98] Eduardo Massad e Oswaldo P. Forattini. Modelling the temperature sensitivity of some physiological parameters of epidemiologic significance. Ecosystem Health, 4(2):119-129, 1998. 5,59

[Mul90] James S. Muldowney. Compound matrices and ordinary differential equations. Rocky Mountain Journal of Mathematics, 20(4), 1990. 22, 26, 76, 77

[Nis06] Hiroshi Nishiura. Mathematical and statistical analyses of the spread of dengue. Dengue Bulletin, 2006. 4

[Nov08] Artem S. Novozhilov. Heterogeneous susceptibles-infectives model: Mechanistic derivation of the power law transmission function. arXiv preprint arXiv:0809.1578, 2008. 5

[NR92] Elizabeth A. C. Newton e Paul Reiter. A model of the transmission of dengue fever with an evaluation of the impact of ultra-low volume (ulv) insecticide applications on dengue epidemics. The American Journal of Tropical Medicine and Hygiene, 47(6):709-720, 1992. 58

[NS00] Gideon A. Ngwa e William S. Shu. A mathematical model for endemic malaria with variable human and mosquito populations. Mathematical and Computer Modelling, 32(7):747-763, 2000. 58,60

[Org] World Health Organization. Dengue. http://www.who.int/denguecontrol/en/. Acessado: 30-05-2016. xvii, xviii

[Sar14] Larissa Marques Sartori. Métodos para resolução de edos stiff resultantes de modelos químicos atmosféricos. Dissertação de Mestrado, Universidade de São Paulo, 2014. xix, 58

[SB13] Josef Stoer e Roland Bulirsch. Introduction to numerical analysis, volume 12. Springer Science \& Business Media, 2013. 58

[SD95] Lisa Sattenspiel e Klaus Dietz. A structured epidemic model incorporating geographic mobility among regions. Mathematical biosciences, 128(1):71-91, 1995. 5

$\left[\mathrm{SFM}^{+} 13\right]$ Steven T. Stoddard, Brett M. Forshey, Amy C. Morrison, Valerie A. Paz-Soldan, Gonzalo M. Vazquez-Prokopec, Helvio Astete, Robert C. Reiner, Stalin Vilcarromero, John P. Elder, Eric S. Halsey et al. House-to-house human movement drives dengue virus transmission. Proceedings of the National Academy of Sciences, 110(3):994-999, 2013. 5

[Smi88] Hal L. Smith. Systems of ordinary differential equations which generate an order preserving flow. a survey of results. SIAM review, 30(1):87-113, 1988. 22, 76, 77

[Smi95] Hal L. Smith. Monotone dynamical systems: an introduction to the theory of competitive and cooperative systems, volume 41. Providence, RI:. American Mathematical Society, 1995. 13, 22,76

[Tel79] Jorge Manuel Sotomayor Tello. Lições de equações diferenciais ordinárias. Inst. de matemática pura e aplicada, 1979. 12, 13, 76

[VW10] Emilia Vynnycky e Richard White. An introduction to infectious disease modelling. Oxford University Press, 2010. 6

[WET $\left.{ }^{+} 12\right]$ Amy Wesolowski, Nathan Eagle, Andrew J. Tatem, David L. Smith, Abdisalan M. Noor, Robert W. Snow e Caroline O. Buckee. Quantifying the impact of human mobility on malaria. Science, 338(6104):267-270, 2012. 5

[WH91] Gerhard Wanner e Ernst Hairer. Solving ordinary differential equations II, volume 1. SpringerVerlag, Berlin, 1991. 57 
[WLG ${ }^{+}$09] Pei-Chih Wu, Jinn-Guey Lay, How-Ran Guo, Chuan-Yao Lin, Shih-Chun Lung e Huey-Jen Su. Higher temperature and urbanization affect the spatial patterns of dengue fever transmission in subtropical taiwan. Science of the total Environment, 407(7):2224-2233, 2009. 5

[WLRVdD06] Marjorie J. Wonham, Mark A. Lewis, Joanna Renclawowicz e P. Van den Driessche. Transmission assumptions generate conflicting predictions in host-vector disease models: a case study in west nile virus. Ecology letters, 9(6):706-725, 2006. 5

[ZS94] Hsiu-Rong Zhu e Hal L. Smith. Stable periodic orbits for a class of three dimensional competitive systems. Journal of Differential Equations, 110(1):143-156, 1994. 34, 76 